Algebraic $\& \mathcal{G}$ Geometric Topology

Volume 2 (2002) 665-741

Published: 6 September 2002

\title{
A functor-valued invariant of tangles
}

\author{
Mikhail KhOVANOV
}

\begin{abstract}
We construct a family of rings. To a plane diagram of a tangle we associate a complex of bimodules over these rings. Chain homotopy equivalence class of this complex is an invariant of the tangle. On the level of Grothendieck groups this invariant descends to the Kauffman bracket of the tangle. When the tangle is a link, the invariant specializes to the bigraded cohomology theory introduced in our earlier work.
\end{abstract}

AMS Classification 57M25; 57M27, 16D20, 18G60

Keywords Tangles, Jones polynomial, Kauffman bracket, TQFT, complexes, bimodules

\section{Introduction}

This paper is a sequel to [38] where we interpreted the Jones polynomial as the Euler characteristic of a cohomology theory of links. Here this cohomology theory is extended to tangles.

The Jones polynomial $[30,34]$ is a Laurent polynomial $J(L)$ with integer coefficients associated to an oriented link $L$ in $\mathbb{R}^{3}$. In [38] to a generic plane projection $D$ of an oriented link $L$ in $\mathbb{R}^{3}$ we associated doubly graded cohomology groups

$$
\mathcal{H}(D)=\underset{i, j \in \mathbb{Z}}{\oplus} \mathcal{H}^{i, j}(D)
$$

and constructed isomorphisms $\mathcal{H}^{i, j}\left(D_{1}\right) \cong \mathcal{H}^{i, j}\left(D_{2}\right)$ for diagrams $D_{1}, D_{2}$ related by a Reidemeister move. Isomorphism classes of groups $\mathcal{H}^{i, j}(D)$ are link invariants, therefore. Moreover, the Jones polynomial equals the weighted alternating sum of ranks of these groups:

$$
J(L)=\sum_{i, j}(-1)^{i} q^{j} \operatorname{rk}\left(\mathcal{H}^{i, j}(D)\right) .
$$

The Jones polynomial extends to a functor from the category of tangles to the category of vector spaces. A tangle is a one-dimensional cobordism in $\mathbb{R}^{2} \times[0,1]$ 
between two finite sets of points, called top and bottom endpoints, which lie on the two boundary components of $\mathbb{R}^{2} \times[0,1]$. The functor $J$ takes a plane with $n$ marked points to $V^{\otimes n}$, where $V$ is the two-dimensional irreducible representation of the quantum group $U_{q}\left(\mathfrak{s l}_{2}\right)$. To an oriented tangle $L$ with $n$ bottom and $m$ top endpoints $J$ associates an operator $J(L): V^{\otimes n} \rightarrow V^{\otimes m}$ which intertwines the $U_{q}\left(\mathfrak{s l}_{2}\right)$ action (see [40],[15]).

Another version of $J$ is the functor $J^{\prime}$ from the category of even tangles (tangles with even number of top and bottom endpoints) to the category of vector spaces. We call a tangle with $2 m$ top and $2 n$ bottom endpoints an $(m, n)$-tangle. $J^{\prime}$ takes a plane with $2 n$ marked points to $\operatorname{Inv}(n) \stackrel{\text { def }}{=} \operatorname{Inv}\left(V^{\otimes 2 n}\right)$, the space of $U_{q}\left(\mathfrak{s l}_{2}\right)$-invariants in $V^{\otimes 2 n}$, and an even tangle $L$ to the map $J^{\prime}(L): \operatorname{Inv}(n) \rightarrow$ $\operatorname{Inv}(m)$ which is the restriction of $J(L)$ to the space of invariants. This wellknown construction is explicitly or implicitly stated in $[42,35,15,23]$.

In Sections 2 and 3 we categorify this invariant of tangles, extending the cohomology theory $\mathcal{H}$. Categorification is an informal procedure which turns integers into abelian groups, vector spaces into abelian or triangulated categories, operators into functors between these categories (see [18]). In our case, the Jones polynomial turns into cohomology groups $\mathcal{H}$, the space of invariants $\operatorname{Inv}(n)$ into a triangulated category $\mathcal{K}^{n}$ (the chain homotopy category of complexes of graded modules over a certain ring $H^{n}$ ), and the operator $J^{\prime}(L)$ into the functor from $\mathcal{K}^{n}$ to $\mathcal{K}^{m}$ of tensoring with a complex $\mathcal{F}(L)$ of $\left(H^{m}, H^{n}\right)$-bimodules.

The fundamental object at the center of our construction is the graded ring $H^{n}$, introduced in Section 2.4. The minimal idempotents of $H^{n}$ are in a bijection with crossingless matchings of $2 n$ points, i.e. ways to pair up $2 n$ points on the unit circle by $n$ arcs that lie inside the unit disc and do not intersect. The number of crossingless matchings is known as the $n$th Catalan number and equals to the dimension of $\operatorname{Inv}(n)$. In addition, there is a natural choice of a basis in $\operatorname{Inv}(n)$, called the graphical basis, and a bijection between elements of this basis and crossingless matchings [42], [23].

Various combinatorial properties of the graphical basis of $\operatorname{Inv}(n)$ lift into statements about the ring $H^{n}$ and its category of representations. For instance the Grothendieck group of the category of $H^{n}$-modules is free abelian and has rank equal to the $n$-th Catalan number. We can glue crossingless matchings $a$ and $b$ along the boundary to produce a diagrams of $k$ circles on the 2 -sphere. Indecomposable projective $H^{n}$-modules are in a bijection with crossingless matchings, and the group of homomorphisms from $P_{a}$ to $P_{b}$ (projective modules associated to $a$ and $b$ ) is free abelian of rank $2^{k}$. 
To a one-dimensional cobordism $a$ in $\mathbb{R} \times[0,1]$ (which we call a flat cobordism or a flat tangle) with $2 n$ bottom and $2 m$ top endpoints we associate a graded $\left(H^{m}, H^{n}\right)$-bimodule $\mathcal{F}(a)$, see Section 2.7. To a two-dimensional cobordism $S$ in $\mathbb{R}^{3}$ between two flat cobordisms $a$ and $b$ we associate a homomorphism $\mathcal{F}(a) \rightarrow \mathcal{F}(b)$ of graded bimodules. We get a functor from the category of two-dimensional cobordisms in $\mathbb{R}^{3}$ to the category of $\left(H^{m}, H^{n}\right)$-bimodules and bimodule homomorphisms. Summing over all $n$ and $m$ results in a two-functor (Section 2.9) from the two-category of surfaces with corners embedded in $\mathbb{R}^{3}$ (the Temperley-Lieb two-category, described in Section 2.3) to the two-category of bimodules and bimodule maps.

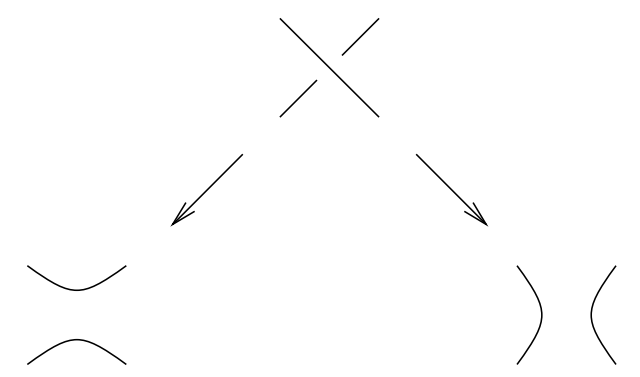

Figure 1: Two resolutions of a crossing

Given a generic plane projection $D$ of an oriented $(m, n)$-tangle $L$, each crossing of $D$ can be "resolved" in two possible ways, as depicted in Figure 1. A plane diagram $D$ with $k$ crossings admits $2^{k}$ resolutions. Each resolution $a$ is a onedimensional cobordism in $\mathbb{R} \times[0,1]$ between the boundary points of $D$, and has a bimodule $\mathcal{F}(a)$ associated to it. There are natural homomorphisms between these bimodules that allow us to arrange all $2^{k}$ of them into a complex, denoted $\mathcal{F}(D)$, as will be explained in Section 3 .

In Section 4 we prove that complexes $\mathcal{F}\left(D_{1}\right)$ and $\mathcal{F}\left(D_{2}\right)$ are chain homotopy equivalent if $D_{1}$ and $D_{2}$ are related by a Reidemeister move. Therefore, the chain homotopy equivalence class of $\mathcal{F}(D)$ is an invariant of a tangle $L$, denoted $\mathcal{F}(L)$. This invariant categorifies the quantum invariant $J^{\prime}(L): \operatorname{Inv}(n) \rightarrow$ $\operatorname{Inv}(m)$, in the following sense.

Let $\mathcal{K}_{P}^{n}$ be the category of bounded complexes of graded projective $H^{n}$-modules up to homotopies. The Grothendieck group $G\left(\mathcal{K}_{P}^{n}\right)$ is a free $\mathbb{Z}\left[q, q^{-1}\right]$-module of rank equal to dimension of $\operatorname{Inv}(n)$. Moreover, there is a natural isomorphism between $G\left(\mathcal{K}_{P}^{n}\right)$ and the $\mathbb{Z}\left[q, q^{-1}\right]$-submodule of $\operatorname{Inv}(n)$ generated by elements of the graphical basis. In particular, for a generic complex number $q$ there is 
an isomorphism

$$
G\left(\mathcal{K}_{P}^{n}\right) \otimes_{\mathbb{Z}\left[q, q^{-1}\right]} \mathbb{C} \cong \operatorname{Inv}(n) .
$$

Tensoring with the complex $\mathcal{F}(D)$, for a plane diagram $D$, can be viewed as a functor from $\mathcal{K}_{P}^{n}$ to $\mathcal{K}_{P}^{m}$. On the Grothendieck groups this functor descends to an operator $G\left(\mathcal{K}_{P}^{n}\right) \rightarrow G\left(\mathcal{K}_{P}^{m}\right)$, equal to $J^{\prime}(L)$ under the isomorphism (3).

When the tangle $L$ is a link, our invariant $\mathcal{F}(L)$ specializes to the bigraded cohomology groups $\mathcal{H}(L)$ of the link $L$, defined in [38]. In detail, a link $L$ is a tangle without endpoints, so that $\mathcal{F}(L)$ is complex of graded $\left(H^{0}, H^{0}\right)$-modules. The ring $H^{0}$ is isomorphic to $\mathbb{Z}$, and $\mathcal{F}(L)$ is just a complex of graded abelian groups, isomorphic to the complex $\mathcal{C}(L)$ defined in [38, Section 7]. $\mathcal{H}(L)$ are its cohomology groups. Thus, we can view rings $H^{n}$ and complexes $\mathcal{F}(L)$ of $\left(H^{m}, H^{n}\right)$-bimodules as an extension of the cohomology theory $\mathcal{H}$.

$\mathcal{F}(L)$ is a relative, or localized, version of cohomology groups $\mathcal{H}$, and their definitions are similar. $\mathcal{F}(L)$, with its $\left(H^{m}, H^{n}\right)$-module structure ignored, is isomorphic to the direct sum of complexes $\mathcal{C}(a L b)$ over all possible ways to close up $L$ into a link by pairing up its top endpoints via a flat $(0, m)$-tangle $a$, and its bottom endpoints via a flat $(n, 0)$-tangle $b$. In particular, the proof of the invariance of $\mathcal{F}(L)$ is nearly identical to that of $\mathcal{H}$. To make the paper self-contained, we repeat some concepts, results and proofs from [38], but often in a more concise form, to prevent us from copying [38] page by page.

The reader who compares this paper with [38] will notice that here we treat the case $c=0$ only. This is done to simplify the exposition. The base ring in [38] was $\mathbb{Z}[c]$. To get the Jones polynomial as the Euler characteristic it suffices to set $c=0$, which results in only finite number of nonzero cohomology groups for each link [38, Section 7]. Generalizing the results of this paper from $\mathbb{Z}$ to $\mathbb{Z}[c]$ does not represent any difficulty.

In a sequel to this paper we will extend the invariant $\mathcal{F}(L)$ to an invariant of tangle cobordisms. The invariant of a cobordism will be a homotopy class of homomorphisms between complexes of bimodules associated to boundaries of the tangle cobordism, or, equvalently, the invariant is a natural transformation between the functors associated to the boundaries of that cobordism.

In the forthcoming joint work with Tom Braden [13] we will relate rings $H^{n}$ with categories of perverse sheaves on Grassmannians. Tom Braden [12] proved that the category of perverse sheaves on the Grassmannian of $k$-dimensional planes in $\mathbb{C}^{k+l}$ (sheaves are assumed smooth along the Schubert cells) is equivalent to the category of modules over a certain algebra $A_{k, l}$, which he explicitly described via generators and relations. We will show that $A_{k, l}$ is isomorphic to 
a subquotient ring of $H^{k+l} \otimes_{\mathbb{Z}} \mathbb{C}$. This result is a step towards the conjecture [38, page 365], [8] that the cohomology theory $\mathcal{H}$ is encoded in parabolic blocks of highest weight categories of $\mathfrak{s l}_{n}$-modules, over all $n$.

Section 6, written rather informally, explains our views on the question: what sort of algebraic structures describe quantum topology in dimension four? In other words, we want to find a combinatorial description and underlying categorical structures of Floer-Donaldson-Seiberg-Witten invariants and any similar invariants of 4-manifolds. This problem was considered by Louis Crane and Igor Frenkel [18] (see also [5], for instance).

An $n$-dimensional topological quantum field theory (TQFT) is, roughly, a tensor functor from the category of $n$-dimensional cobordisms between closed oriented $(n-1)$-manifold to an additive tensor category. Interesting examples are known in dimensions 3 and 4 . In dimension 3 there is the Witten-ReshetikhinTuraev TQFT (constructed from representations of quantum $\mathfrak{s l}_{2}$ at a root of unity) and its generalizations to arbitrary complex simple Lie algebras. In dimension 4 there are Floer-Donaldson and Seiberg-Witten TQFTs. Twodimensional TQFTs are in a bijection with Frobenius algebras. As suggested by Igor Frenkel, we believe that no interesting examples of TQFTs exist beyond dimension 4 (TQFTs constructed from fundamental groups and other algebraic topology structures do not qualify, since the quantum flavor is missing).

It is more complicated to define a TQFT for manifolds with corners. For short, we will call it a TQFT with corners. $n$-dimensional manifolds with corners constitute a 2-category $\mathbb{M C}_{n}$ whose objects are closed oriented $(n-2)$-manifolds, 1-morphisms are $(n-1)$-dimensional cobordisms between $(n-2)$-manifolds, and 2-morphisms are $n$-dimensional cobordisms between $(n-1)$-cobordisms. A TQFT with corners is a 2-functor from $\mathbb{M C}_{n}$ to the 2-category $\mathbb{A C}$ of additive categories. Objects of $\mathbb{A} \mathbb{C}$ are additive categories, 1-morphisms are exact functors and 2-morphisms are natural transformations. Examples have been worked out in dimension 3 only, where the Witten-Reshetikhin-Turaev TQFT extends to a TQFT with corners.

There are indications that Floer-Donaldson and Seiberg-Witten 4D TQFT extend to TQFTs with corners. According to Fukaya [24], the category associated to a connected closed surface in the Floer-Donaldson TQFT with corners should be the $A_{\infty}$-category of lagrangian submanifolds in the moduli space of flat $S O(3)$-connections over the surface. Kontsevich conjectured that $A_{\infty}$ categories of lagrangian submanifolds in symplectic manifolds can be made into $A_{\infty}$-triangulated categories, which, in turn, are $A_{\infty}$-equvalent to triangulated categories. Putting symplectic topology and $A_{\infty}$-categories aside, here is how we see the problem. 
Problem Construct 4-dimensional TQFTs, including the ones of Floer-Donaldson and Seiberg-Witten, and their extensions to 4-dimensional TQFTs with corners. Construction should be combinatorial and explicit. To a closed oriented surface $K$ (decorated, if necessary, by homology classes, spin structure, etc) associate a triangulated category $F(K)$. To a suitably decorated 3 -cobordism $M$ associate an exact functor $F(M): F\left(\partial_{0} M\right) \rightarrow F\left(\partial_{1} M\right)$. To a suitably decorated 4-cobordism $N$ associate a natural transformation of functors $F(N): F\left(\partial_{0} N\right) \rightarrow F\left(\partial_{1} N\right)$.

$F$ should be a 2-functor from the 2-category of oriented and decorated 4manifolds with corners to the 2-category of triangulated categories. $F$ should be tensor, in appropriate sense.

Categories $F(K)$ should be described explicitly, for instance, as derived categories of modules over differential graded algebras, the latter given by generators and relations. The answer is likely to be even fancier, possibly requiring $\mathbb{Z}_{m}$ graded rather than $\mathbb{Z}$-graded complexes, or sophisticated localizations, but still as clear-cut as triangulated categories could be. Functors $F(M)$ and natural transformations $F(N)$ should be given equally explicit descriptions.

Why do we want categories $F(K)$ to be additive? Let $M_{1}$ and $M_{2}$ be 3 manifolds, each with boundary diffeomorphic to $K$. We can glue $M_{1}$ and $M_{2}$ along $K$ into a closed 3-manifold $M=M_{1} \cup_{K}\left(-M_{2}\right)$. The invariant $F(M)$ of a closed 3-manifold is going to be a vector space or, may be, an abelian group (think of Floer homology groups). On the other hand, $F(M) \cong$ $\operatorname{Hom}_{F(K)}\left(F\left(M_{1}\right), F\left(M_{2}\right)\right)$. Varying $M_{1}$ and $M_{2}$ we get a number of objects in $F(K)$. These objects will, in some sense, generate $F(K)$ (if not, just pass to the subcategory generated by these objects). The set of morphisms between each pair of these objects has an abelian group structure. Introducing formal direct sums of objects, if necessary, we can extend additivity from morphisms to objects. It is thus plausible to expect $F(K)$ to be additive categories.

Why do we expect $F(K)$ to be triangulated? Typical examples of additive categories are either abelian categories and their subcategories or triangulated categories. The mapping class group of the surface $K$ acts on $F(K)$. Automorphism groups of abelian categories have little to do with mapping class groups of surfaces. Triangulated categories occasionally have large automorphisms groups, and sometimes contain braid groups as subgroups (see Section 6.5). The braid group isn't that far off from the mapping class group of a closed surface. This observation quickly biases us away from abelian and towards triangulated categories. 
In the 2-category $\mathbb{M C}_{n}$ of $n$-cobordisms with corners an $(n-1)$-cobordism $M$ from $N_{0}$ to $N_{1}$ has a biadjoint cobordism $W$, which is $M$ considered as a cobordism from $N_{1}$ to $N_{0}$. Consequently, for any 2-functor $F$ from $\mathbb{M C}_{n}$ to the 2-category of all small categories, the 1-morphism $F(M)$ has a biadjoint. In other words, the functor $F(W)$ is left and right adjoint to $F(M)$. A functor which has a biadjoint is called a Frobenius functor.

This property hardly ever surfaced for 3-dimensional TQFT with corners, since in main examples the categories $F(K)$ were semisimple and functors between them were Frobenius for the obvious reason. Not so in dimension 4 , where semisimple categories are out of favor.

These observations lead to the following heuristic principle:

Categories associated to surfaces in 4-dimensional TQFTs with corners will be triangulated categories with large automorphism groups and admitting many Frobenius functors.

Among prime suspects are derived categories of

- the category $\mathcal{O}$,

- categories of modules over Frobenius algebras,

- categories of coherent sheaves on Calabi-Yau manifolds.

We believe that carefully picked categories from these classes of derived categories will give rise to invariants of 2-knots and knot cobordisms, while invariants of 4-manifolds will emerge from less traditional triangulated categories.

Acknowledgements Section 5.1 was inspired by a conversation with Raphaël Rouquier. The observation that the braid group acts on derived categories of sheaves on partial flag varieties (see Section 6.5) emerged during a discussion with Tom Braden.

\section{A bimodule realization of the Temperley-Lieb two- category}

\section{$2.1 \quad \operatorname{Ring} \mathcal{A}$ and two-dimensional cobordisms}

All tensor products are over the ring of integers unless specified otherwise. Let $\mathcal{A}$ be a free abelian group of rank 2 spanned by 1 and $X$. We make $\mathcal{A}$ into a 
graded abelian group by assigning degree -1 to 1 and degree 1 to $X$. Introduce a commutative associative multiplication map $m: \mathcal{A} \otimes \mathcal{A} \rightarrow \mathcal{A}$ by

$$
\mathbf{1}^{2}=\mathbf{1}, \quad \mathbf{1} X=X \mathbf{1}=X, \quad X^{2}=0 .
$$

$m$ is a graded map of degree 1 . Define the unit map $\iota: \mathbb{Z} \rightarrow \mathcal{A}$ by $\iota(1)=\mathbf{1}$. Define the trace map $\epsilon: \mathcal{A} \rightarrow \mathbb{Z}$ by

$$
\epsilon(\mathbf{1})=0, \quad \epsilon(X)=1
$$

$\mathcal{A}$ is a commutative ring with a nondegenerate trace form. Such a ring defines a two-dimensional topological quantum field theory - a functor from the category $\mathcal{M}$ of oriented cobordisms between one-manifolds to the category of abelian groups and group homomorphisms [1], [6, Section 4.3].

In our case, this functor, which we will call $\mathcal{F}$ (following the notation from [38, Section 7.1]), associates abelian group $\mathcal{A}^{\otimes k}$ to a disjoint union of $k$ circles. To elementary cobordisms $S_{2}^{1}, S_{0}^{1}, S_{1}^{0}$, depicted in figure $2, \mathcal{F}$ associates maps $m, \iota$ and $\epsilon$, respectively (here $S_{j}^{i}$ is the connected cobordism of the minimal possible genus between $j$ and $i$ circles).
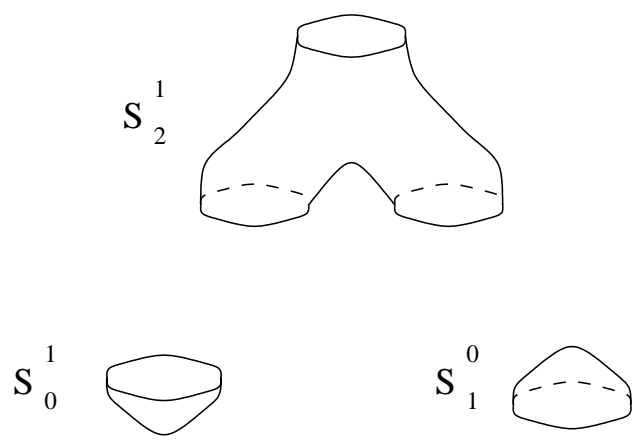

Figure 2: Elementary cobordisms

To a 2-sphere with 3 holes, considered as a cobordism from one circle to two circles (this is different from the surface $S_{2}^{1}$, which we view as a cobordism from two circles to one circle), the functor $\mathcal{F}$ associates the map

$$
\Delta: \mathcal{A} \rightarrow \mathcal{A}^{\otimes 2}, \quad \Delta(\mathbf{1})=\mathbf{1} \otimes X+X \otimes \mathbf{1}, \quad \Delta(X)=X \otimes X .
$$

The map $\mathcal{F}(S)$ of graded abelian groups, associated to a surface $S$, is a graded map of degree minus the Euler characteristic of $S$ :

$$
\operatorname{deg}(\mathcal{F}(S))=-\chi(S) .
$$

The ring $\mathcal{A}$ is essential for the construction ([38, Section 7]) of the link cohomology theory $\mathcal{H}$. In [38] this ring was equipped with the opposite grading. 
In this paper we invert the grading to make the ring $H^{n}$ (defined later, in Section 2.4, and central to our considerations) positively graded rather than negatively graded.

Given a graded abelian group $G=\underset{k \in \mathbb{Z}}{\oplus} G_{k}$, denote by $G\{n\}$ the abelian group obtained by raising the grading of $G$ by $n$ :

$$
G\{n\}=\underset{k \in \mathbb{Z}}{\oplus} G\{n\}_{k}, \quad G\{n\}_{k}=G_{k-n} .
$$

Remark In [38] $\{n\}$ denotes the downward rather than the upward shift by $n$ in the grading.

We will be using functor $\mathcal{F}$ in the following situation. Let $\mathcal{E S}$ be the category of surfaces embedded in $\mathbb{R}^{2} \times[0,1]$. Objects of $\mathcal{E S}$ are smooth embeddings of closed one-manifolds into $\mathbb{R}^{2}$. A morphism is a compact surface $S$ smoothly embedded in $\mathbb{R}^{2} \times[0,1]$ such that the boundary of $S$ lies in the boundary of $\mathbb{R}^{2} \times[0,1]$, and $S$ is tubular near its boundary, i.e., for some small $\delta>0$,

$$
\begin{aligned}
S \cap\left(\mathbb{R}^{2} \times[0, \delta]\right) & =\left(\partial_{0} S\right) \times[0, \delta], \\
S \cap\left(\mathbb{R}^{2} \times[1-\delta, 1]\right) & =\left(\partial_{1} S\right) \times[1-\delta, 1],
\end{aligned}
$$

where we denoted

$$
\begin{array}{ll}
\partial_{0} S & \stackrel{\text { def }}{=} \partial S \cap\left(\mathbb{R}^{2} \times\{0\}\right), \\
\partial_{1} S \stackrel{\text { def }}{=} \partial S \cap\left(\mathbb{R}^{2} \times\{1\}\right) .
\end{array}
$$

We will call a surface $S \subset \mathbb{R}^{2} \times[0,1]$ satisfying these conditions a slim surface. The tubularity condition is imposed to make easy the gluing of slim surfaces along their boundaries. We view a slim surface $S$ as a cobordism from $\partial_{0} S$ to $\partial_{1} S$, and as a morphism in $\mathcal{E} \mathcal{S}$. Two morphisms are equal if slim surfaces representing them are isotopic relative to the boundary. Morphisms are composed by concatenating the surfaces along the boundary.

We now construct a functor from $\mathcal{E S}$ to the category $\mathcal{M}$ of oriented twodimensional cobordisms (no longer embedded in $\mathbb{R}^{2} \times[0,1]$ ). This functor forgets the embedding of $S$ into $\mathbb{R}^{2} \times[0,1]$. Before the embedding is forgotten, it is used to orient $S$, as follows.

First, any object $C$ of $\mathcal{E S}$ (a closed one-manifold embedded in $\mathbb{R}^{2}$ ) comes with a natural orientation. Namely, we orient a component $C^{\prime}$ of $C$ counterclockwise if even number of components of $C$ separate $C^{\prime}$ from the "infinite" point of $\mathbb{R}^{2}$. Otherwise orient $C^{\prime}$ clockwise. A clarifying example is depicted in Figure 3.

A slim surface $S$ admits the unique orientation that induces natural orientations of its boundaries $\partial_{0} S$ and $\partial_{1} S$. An orientation of a component $S^{\prime}$ of $S$ depends 


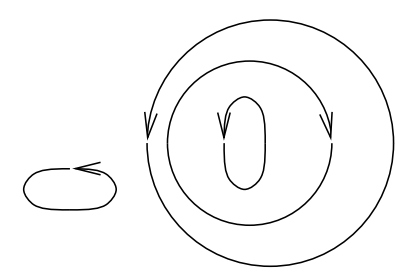

Figure 3: Orientations of objects of $\mathcal{E S}$

on the parity of the number of components of $S$ that separate $S^{\prime}$ from the infinity in $\mathbb{R}^{2} \times[0,1]$. We call this orientation the natural orientation of $S$.

The natural orientation of slim surfaces and their boundaries behaves well under compositions, and can be used to define a functor from $\mathcal{E S}$ to the category $\mathcal{M}$ of oriented two-cobordisms. This functor forgets the embedding but keeps the natural orientation of slim surfaces and their boundaries. Composing the forgetful functor with $\mathcal{F}$, which is a functor from $\mathcal{M}$ to graded abelian groups, we get a functor from the category of slim surfaces to the category of graded abelian groups and graded maps. We will denote this functor also by $\mathcal{F}$.

\subsection{Flat tangles and the Temperley-Lieb category}

The Temperley-Lieb category $\mathcal{T} \mathcal{L}$ is a category with objects-collections of marked points on a line and morphisms-cobordisms between these collections of points. In this paper we restrict to the case when the number of marked points is even. The objects of the Temperley-Lieb category are nonnegative integers, $n \geq 0$, presented by a horizontal line lying in a Euclidean plane, with $2 n$ points marked on this line. For convenience, from now on we require that the $x$-coordinates of these marked points are $1,2, \ldots, 2 n$. A morphism from $n$ and $m$ is a smooth proper embedding of a disjoint union of $n+m$ arcs and a finite number of circles into $\mathbb{R} \times[0,1]$ such that the boundary points or arcs map bijectively to the $2 n$ marked points on $\mathbb{R} \times\{0\}$ and $2 m$ marked points on $\mathbb{R} \times\{1\}$. In addition, we require that around the endpoints the arcs are perpendicular to the boundary of $\mathbb{R} \times[0,1]$ (this ensures that the concatenation of two such embeddings is a smooth embedding). An embedding with this property will be called a flat tangle, or a flat $(m, n)$-tangle. We define morphisms in the Temperley-Lieb category $\mathcal{T} \mathcal{L}$ as flat tangles up to isotopy. In general, we will distinguish between equal and isotopic flat tangles. The embedding of the empty 1-manifold is a legitimate $(0,0)$-flat tangle. An example of a flat tangle is depicted in Figure 4. 


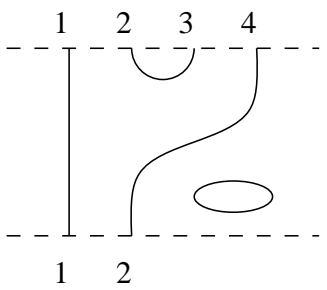

Figure 4: A flat $(2,1)$-tangle

Given a flat $(m, n)$-tangle $a$ and a flat $(k, m)$-tangle $b$, define the composition $b a$ as the concatenation of $b$ and $a$. In details, we identify the top boundary of $a$ with the lower boundary of $b$ so that the $2 m$ marked points on each of these boundary components match. The result is a configuration of arcs and circles in $\mathbb{R} \times[0,2]$. We rescale it along the second coordinate to get a configuration in $\mathbb{R} \times[0,1]$. The resulting diagram is a flat $(k, n)$-tangle.

Denote by $\operatorname{Vert}_{2 n}$ the vertical embedding of $2 n$ arcs (i.e. the $i$-th arc embeds as the segment $(i, y), 0 \leq y \leq 1)$. This flat $(n, n)$-tangle is the identity morphism from $n$ to $n$.

Denote by $\widehat{B}_{n}^{m}$ the space of flat tangles with $2 n$ bottom and $2 m$ top points. Let

$$
W: \widehat{B}_{n}^{m} \rightarrow \widehat{B}_{m}^{n}
$$

be the involution of the space of flat tangles sending a flat tangle to its reflection about the line $\mathbb{R} \times\left\{\frac{1}{2}\right\}$. An example is depicted in Figure 5 .
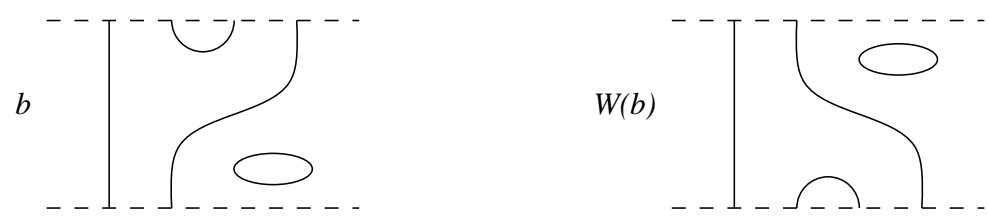

Figure 5: Involution $W$

Choose a base point in each connected component of $\widehat{B}_{n}^{m}$ that consists of embeddings without circles. Denote the set of base points by $B_{n}^{m}$. We pick the base points so that $W\left(B_{n}^{m}\right)=B_{m}^{n}$ for all $n$ and $m$. Note that the cardinality of $B_{n}^{m}$ is the $(n+m)$ th Catalan number. Let $\mathrm{rm}: \widehat{B}_{n}^{m} \longrightarrow B_{n}^{m}$ be the map that removes all circles from a diagram $b \in \widehat{B}_{n}^{m}$, producing a diagram $c$, and assigns to $b$ the representative of $c$ in $B_{n}^{m}$ (the unique flat $(m, n)$-tangle in $B_{n}^{m}$ isotopic to $c$ ). 
Denote the set $B_{0}^{n}$ by $B^{n}$. An element in $B^{n}$ represents an isotopy class of pairwise disjoint embeddings of $n$ arcs in $\mathbb{R} \times[0,1]$ connecting in pairs $2 n$ points on $\mathbb{R} \times\{1\}$. Thus, elements of $B^{n}$ are crossingless matchings of $2 n$ points.

Define $\mathcal{L} \mathcal{T} \mathcal{L}$, the linear Temperley-Lieb category, as a category with objectsnonnegative integers, and morphisms from $n$ to $m$-formal linear combinations of elements of $B_{n}^{m}$ with coefficients in $\mathbb{Z}\left[q, q^{-1}\right]$. The composition of morphisms is $\mathbb{Z}\left[q, q^{-1}\right]$-linear, and if $a \in B_{n}^{m}, b \in B_{m}^{k}$, define their composition as $\left(q+q^{-1}\right)^{i} \mathrm{rm}(b a)$, where $i$ is the number of circles in $b a$. In other words, we concatenate $b$ and $a$ and then remove all circles from $b a$, multiplying the diagram by $q+q^{-1}$ each time we remove a circle.

Define the linearization functor

$$
\operatorname{lin}: \mathcal{T} \mathcal{L} \longrightarrow \mathcal{L} \mathcal{T} \mathcal{L}
$$

as the identity on objects, and $\operatorname{lin}(a)=\left(q+q^{-1}\right)^{i} \operatorname{rm}(a)$, where $i$ is the number of circles in $a$.

\subsection{The Temperley-Lieb 2-category}

Let $a, b \in \widehat{B}_{n}^{m}$. An admissible cobordism between flat tangles $a$ and $b$ is a surface $S$ smoothly and properly embedded in $\mathbb{R} \times[0,1] \times[0,1]$ subject to conditions

$$
\begin{aligned}
S \cap(\mathbb{R} \times[0,1] \times[0, \delta]) & =a \times[0, \delta] \\
S \cap(\mathbb{R} \times[0,1] \times[1-\delta, 1]) & =b \times[1-\delta, 1] \\
S \cap(\mathbb{R} \times[0, \delta] \times[0,1]) & =\{1,2, \ldots, 2 n\} \times[0, \delta] \times[0,1]) \\
S \cap(\mathbb{R} \times[1-\delta, 1] \times[0,1]) & =\{1,2, \ldots, 2 m\} \times[1-\delta, 1] \times[0,1])
\end{aligned}
$$

for some small $\delta>0$. The first condition says that $S$ contains $a$ in its boundary, moreover, near $a$, the surface $S$ is the direct product of $a$ and the inverval $[0, \delta]$. The second condition gives a similar requirement on the opposite part of $S$ 's boundary. The conditions are imposed to make gluing of two surfaces along a common boundary easy.

The boundary of $S$ consists of $a, b$ and $2(n+m)$ intervals, of which $2 n$ lie in the plane $\mathbb{R} \times\{0\} \times[0,1]$ and remaining $2 m$ in $\mathbb{R} \times\{1\} \times[0,1]$. Conditions (9) and (10) describe these $n+m$ segments explicitly. Notice that the corners of $S$ are in a one-to-one correspondence with the endpoints of $a$ and $b$. It is convenient to present $S$ by a sequence of its cross-sections with planes $\mathbb{R} \times[0,1] \times\{t\}$ for several values of $t \in[0,1]$. See Figure 6 for an example. The first frame depicts 


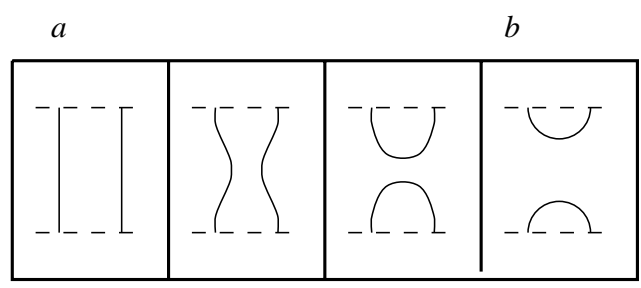

Figure 6: Cross-sections of a cobordism

$a$ (case $t=0)$, the last frame depicts $b$ (case $t=1)$. The two dashed lines in each frame show the boundary of $\mathbb{R} \times[0,1] \times\{t\}$.

If $S$ is an admissible cobordism from $a$ to $b$, let $\partial_{0} S=a, \partial_{1} S=b$. The height function $f: S \rightarrow[0,1]$ of $S$ is the projection on the third factor in the direct product $\mathbb{R} \times[0,1] \times[0,1]$. In particular, $f^{-1}(0)=\partial_{0} S$ and $f^{-1}(1)=\partial_{1} S$.

An admissible cobordism will also be called an admissible surface, and a cobordism between flat tangles. Given an admissible cobordism $S_{1}$ from $a$ to $b$ and an admissible cobordism $S_{2}$ from $b$ to $c$, we can concatenate $S_{1}$ and $S_{2}$ (glue them along their common boundary $b$ ) to get an admissible cobordism, denoted $S_{2} \circ S_{1}$, from $a$ to $c$.

Admissible cobordisms admit another kind of composition. Let $a, b \in \widehat{B}_{n}^{m}$ and $c, d \in \widehat{B}_{m}^{k}$. Let $S_{1}$ be an admissible cobordism from $a$ to $b$ and $S_{2}$ an admissible cobordism from $c$ to $d$. Then we can compose $S_{1}$ and $S_{2}$ to obtain an admissible cobordism, denoted $S_{2} S_{1}$, from $c a$ to $d b$.

Two admissible surfaces are called equivalent, or isotopic, if there is an isotopy from one to the other through admissible surfaces, rel boundary.

A slim surface is the same as an admissible cobordism between flat (0,0)-tangles.

Define the 2-category $\mathbb{T L}$ as a 2-category with objects-nonnegative integers, one-morphisms from $n$ to $m$-flat $(m, n)$-tangles and two-morphisms from $a$ to $b$, where $a, b$ are flat $(m, n)$-tangles - isotopy classes of admissible cobordisms from $a$ to $b$. This 2-category is defined and discussed at length in [16]. We only stress here the difference between morphisms in the category $\mathcal{T} \mathcal{L}$ and 1 morphisms in the two-category $\mathbb{T L}$. The morphisms in $\mathcal{T} \mathcal{L}$ are isotopy classes of flat tangles, equivalently, the morphisms from $n$ to $m$ are connected components of the space $\widehat{B}_{n}^{m}$. One-morphisms in $\mathbb{T L}$ are flat tangles (points of $\widehat{B}_{n}^{m}$ ). Consequently, the composition of one-morphisms in $\mathbb{T L}$ is not strictly associative. If $c, b, a$ are composable 1-morphisms, the compositions $(c b) a$ and $c(b a)$ represent different plane diagrams, so that these 1-morphisms are different. 
The plane diagrams are isotopic, though, and to an isotopy there is associated an admissible surface that defines a 2-morphism from $(c b) a$ and $c(b a)$. This 2-morphism is invertible, and the 1-morphisms $(c b) a$ and $c(b a)$ are isomorphic.

Define the Euler-Temperley-Lieb 2-category $\mathbb{E} \mathbb{T} L$ as a 2-category with objects $n$ for $n \geq 0$, with 1 -morphisms pairs $(a, j)$ where $a$ is a 1-morphism in $\mathbb{T L}$ (a flat tangle) and $j$ an integer. 2-morphisms from $\left(a, j_{1}\right)$ to $\left(b, j_{2}\right)$ are isotopy classes of admissible surfaces $S$ with $\partial_{0} S=a, \partial_{1} S=b$ and

$$
\chi(S)=n+m+j_{2}-j_{1}
$$

(recall that $\chi$ denotes the Euler characteristic).

Given composable flat tangles $a$ and $b$, we define the composition $(a, j)(b, k)$ as $(a b, j+k)$. Earlier we described two possible ways to compose admissible surfaces. Equation (11) ensures consistency for these three kinds of composition of 1- and 2-morphisms, so that $\mathbb{E} \mathbb{T L}$ is indeed a 2-category.

The forgetful functor $\mathbb{E} \mathbb{T L} \longrightarrow \mathbb{T L}$ takes a 1-morphism $(a, j)$ of $\mathbb{E} \mathbb{T L}$ to the 1-morphism $a$ of $\mathbb{T L}$.

\subsection{The ring $H^{n}$}

In this section we define a finite-dimensional graded $\operatorname{ring} H^{n}$, for $n \geq 0$. As a graded abelian group, it decomposes into the direct sum

$$
H^{n}=\underset{a, b}{\oplus} b\left(H^{n}\right)_{a}
$$

where $a, b \in B^{n}$ and

$$
{ }_{b}\left(H^{n}\right)_{a} \stackrel{\text { def }}{=} \mathcal{F}(W(b) a)\{n\} .
$$

Since $a \in B^{n}$ and $W(b) \in B_{n}^{0}$, their composition $W(b) a$ belongs to $B_{0}^{0}$, and is a disjoint union of circles embedded into the plane. Therefore, we can apply the functor $\mathcal{F}$ to $W(b) a$ and obtain $\mathcal{A}^{\otimes k}$ where $k$ is the number of circles in $W(b) a$. Recall that $\{n\}$ denotes the upward shift by $n$ in the grading.

Defining the multiplication in $H^{n}$ is our next task. First, we set $u v=0$ if $u \in{ }_{d}\left(H^{n}\right)_{c}, v \in{ }_{b}\left(H^{n}\right)_{a}$ and $c \neq b$. Second, the multiplication maps

$$
{ }_{c}\left(H^{n}\right)_{b} \otimes{ }_{b}\left(H^{n}\right)_{a} \longrightarrow{ }_{c}\left(H^{n}\right)_{a}
$$

are given as follows. $b W(b)$, for $b \in B^{n}$, is the composition of the mirror image of $b$ with $b$, see Figure 7 for an example. 

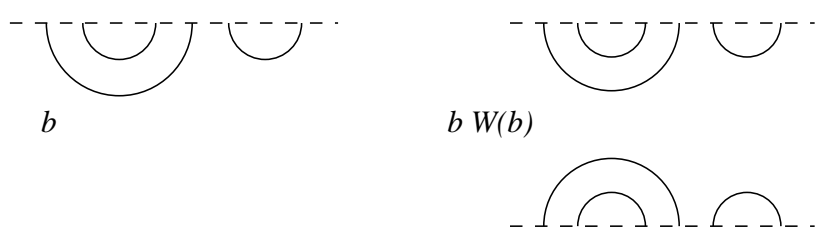

Figure 7: A cobordism $b$ and $b W(b)$

Let $S(b)$ be an admissible surface in $\mathbb{R} \times[0,1] \times[0,1]$ with

$$
\partial_{0} S(b)=b W(b), \quad \partial_{1} S(b)=\text { Vert }_{2 n},
$$

such that $S(b)$ is diffeomorphic to a disjoint union of $n$ discs. In other words, $S(b)$ is the "simplest" cobordism between $b W(b)$ and Vert $2 n$ (recall that Vert ${ }_{2 n}$ denotes the diagram made of $2 n$ vertical segments). $S(b)$ can be arranged to have $n$ saddle points and no other critical points relative to the height function. A clarifying example is depicted in Figure 8 where we present $S(b)$ by a sequence of its intersections with planes $\mathbb{R} \times[0,1] \times\{t\}$, for five distinct values of $t \in[0,1]$. The first frame shows $\partial_{0} S(b)=b W(b)$, the last (frame number 5) shows $\partial_{1}(S(b))=$ Vert $_{2 n}$.
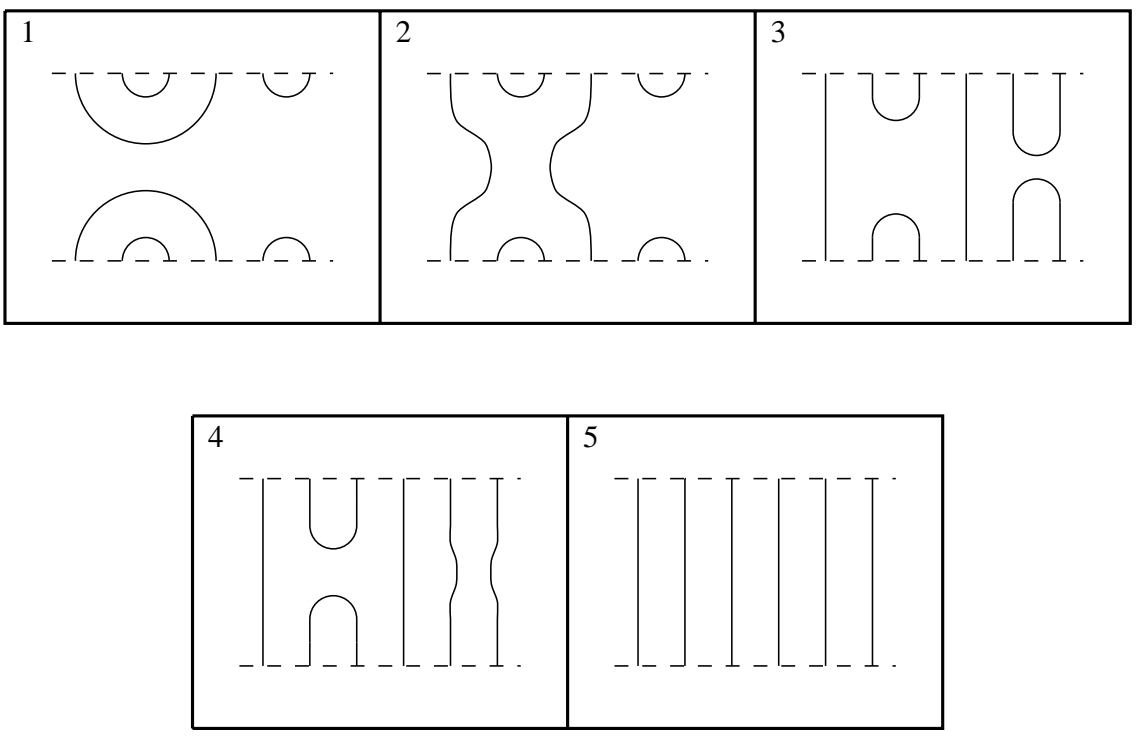

Figure 8: Cobordism $S(b)$

For $a, b, c \in B^{n}$ define a cobordism from $W(c) b W(b) a$ to $W(c) a$ by composing cobordism $S(b)$ with the identity cobordisms from $a$ to itself and from $W(c)$ 
to itself:

$$
W(c) b W(b) a \stackrel{I d_{W(c)} S(b) I d_{a}}{\longrightarrow} W(c) a .
$$

This cobordism is a slim surface and induces a homomorphism of graded abelian groups

$$
\mathcal{F}(W(c) b W(b) a) \longrightarrow \mathcal{F}(W(c) a) .
$$

Since $W(c) b W(b) a$ is the composition of $W(c) b$ and $W(b) a$, both of which consist only of closed circles, we have a canonical isomorphism

$$
\mathcal{F}(W(c) b W(b) a) \cong \mathcal{F}(W(c) b) \otimes \mathcal{F}(W(b) a)
$$

and homomorphism (14) can be written as

$$
\mathcal{F}(W(c) b) \otimes \mathcal{F}(W(b) a) \longrightarrow \mathcal{F}(W(c) a)
$$

The surface underlying cobordism (13) has Euler characteristic $(-n)$, so that (15) has degree $n$ and after shifting we get a grading-preserving map

$$
\mathcal{F}(W(c) b)\{n\} \otimes \mathcal{F}(W(b) a)\{n\} \longrightarrow \mathcal{F}(W(c) a)\{n\}
$$

We define the multiplication

$$
m_{c, b, a}:{ }_{c}\left(H^{n}\right)_{b} \otimes{ }_{b}\left(H^{n}\right)_{a} \longrightarrow{ }_{c}\left(H^{n}\right)_{a}
$$

to be (16), i.e., the diagram below is commutative

$$
\begin{array}{ccc}
{ }_{c}\left(H^{n}\right)_{b} \otimes{ }_{b}\left(H^{n}\right)_{a} & \stackrel{m_{c, b, a}}{\longrightarrow} & { }_{c}\left(H^{n}\right)_{a} \\
\downarrow \cong & \\
\mathcal{F}(W(c) b)\{n\} & \otimes \mathcal{F}(W(b) a)\{n\} & \stackrel{(16)}{\longrightarrow} \mathcal{F}(W(c) a)\{n\}
\end{array}
$$

where the vertical arrows are given by (12).

Maps $m_{c, b, a}$, as we vary $a, b$ and $c$ over elements of $B^{n}$, define a gradingpreserving multiplication in $H^{n}$. Associativity of this multiplication follows from functoriality of $\mathcal{F}$.

The elements $1_{a} \in{ }_{a}\left(H^{n}\right)_{a}$, defined as $\mathbf{1}^{\otimes n}\{n\} \in \mathcal{A}^{\otimes n}\{n\} \cong{ }_{a}\left(H^{n}\right)_{a}$, are idempotents of $H^{n}$. Namely, $1_{a} x=x$ for $x \in{ }_{a}\left(H^{n}\right)_{b}$ and $1_{a} x=0$ for $x \in$ ${ }_{c}\left(H^{n}\right)_{b}, c \neq a$. Similarly, $x 1_{a}=x$ for $x \in{ }_{b}\left(H^{n}\right)_{a}$ and $x 1_{a}=0$ for $x \in$ ${ }_{b}\left(H^{n}\right)_{c}, c \neq a$. Adding up these idempotents, we obtain the unit $1 \in H^{n}$ :

$$
1=\sum_{a \in B^{n}} 1_{a}
$$

To sum up, we have: 
Proposition 1 Structures, described above, make $H^{n}$ into a $\mathbb{Z}_{+}$-graded associative unital ring.

To acquaint ourselves better with the ring $H^{n}$, we next examine it for $n=$ $0,1,2$.

$n=0$. The ring $H^{0}$ is isomorphic to $\mathbb{Z}$, since $B^{0}$ contains only the empty diagram, and the functor $\mathcal{F}$ applied to the empty diagram produces $\mathbb{Z}$.
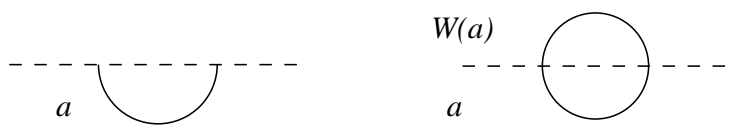

Figure 9: The diagram $a$ in $B^{1}$ and the composition $W(a) a$

$n=1$. There is only one diagram in $B^{1}$, depicted in Figure 9. The composition $W(a) a$ is a circle (see Figure 9), so that

$$
H^{1}={ }_{a}\left(H^{1}\right)_{a}=\mathcal{F}(W(a) a)\{1\}=\mathcal{A}\{1\}
$$

(the first equality holds since $a$ is the only element in $B^{1}$ ). The multiplication in $H^{1}$ is induced via the functor $\mathcal{F}$ by the cobordism $S_{2}^{1}$ (see section 2.1) between two circles (representing $W(a) a W(a) a)$ and one circle (representing $W(a) a$ ). Thus, the multiplication in $H^{1}$ is just the multiplication in the algebra $\mathcal{A}$ and, hence, $H^{1}$ is isomorphic to $\mathcal{A}$, with the grading shifted up by 1 (note that the multiplication in $\mathcal{A}$ becomes grading-preserving after this shift in the grading).

$n=2$. The set $B^{2}$ consists of two diagrams (see Figure 10) which we denote
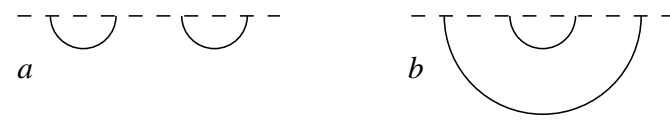

Figure 10: Diagrams in $B^{2}$

by $a$ and $b$, respectively. From Figure 11 we derive that

$$
\begin{aligned}
{ }_{a}\left(H^{2}\right)_{a} & =\mathcal{A}^{\otimes 2}\{2\}, & { }_{b}\left(H^{2}\right)_{a} & =\mathcal{A}\{2\}, \\
{ }_{a}\left(H^{2}\right)_{b} & =\mathcal{A}\{2\}, & { }_{b}\left(H^{2}\right)_{b} & =\mathcal{A}^{\otimes 2}\{2\} .
\end{aligned}
$$

The multiplication table for $H^{2}$ can be easily written down. For instance, the multiplication map ${ }_{a}\left(H^{2}\right)_{b} \times{ }_{b}\left(H^{2}\right)_{a} \rightarrow{ }_{a}\left(H^{2}\right)_{a}$, under the above identifications, becomes the map $\Delta m: \mathcal{A}^{\otimes 2}\{4\} \stackrel{m}{\longrightarrow} \mathcal{A}\{3\} \stackrel{\Delta}{\longrightarrow} \mathcal{A}^{\otimes 2}\{2\}$. 
$W(a) a$<smiles>c1ccccc1</smiles><smiles>c1ccccc1</smiles>

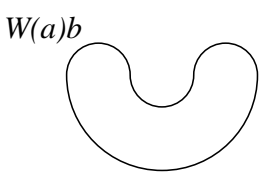

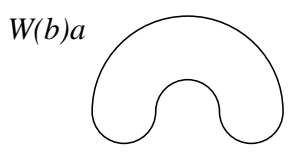

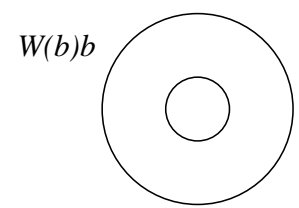

Figure 11: Diagrams $W(a) a, W(a) b, W(b) a$, and $W(b) b$

\subsection{Projective $H^{n}$-modules}

All $H^{n}$-modules and bimodules considered in this paper are assumed graded, unless otherwise specified. All $H^{n}$-module and bimodule homomorphisms are assumed grading-preserving, unless otherwise specified.

Denote by $H^{n}$-mod the category of finitely-generated left $H^{n}$-modules and module maps. The category $H^{n}-\bmod$ is abelian. Since $H^{n}$ is finite over $\mathbb{Z}$, an $H^{n}$-module is finitely generated if and only if it is finitely generated as an abelian group. The functor $\{k\}$ shifts the grading of a module or a bimodule upward by $k$.

$H^{n}$, considered as a left $H^{n}$-module, belongs to $H^{n}$-mod. Let $P_{a}$, for $a \in B^{n}$, be a left $H^{n}$-submodule of $H^{n}$ given by

$$
P_{a}=\underset{b \in B^{n}}{\oplus} b\left(H^{n}\right)_{a}
$$

$H^{n}$ decomposes into a direct sum of left $H^{n}$-modules

$$
H^{n}=\underset{a \in B^{n}}{\oplus} P_{a}
$$

By a projective $H^{n}$-module we mean a projective object of $H^{n}$-mod. Clearly, $P_{a}$ is projective, since it is a direct summand of the free module $H^{n}$. Moreover, $P_{a}$ is indecomposable, since $\mathcal{A}^{\otimes n}\{n\}$, the endomorphism ring of $P_{a}$, has only one idempotent $1_{a}=\mathbf{1}^{\otimes n}\{n\}$. 
Proposition 2 An indecomposable projective $H^{n}$-module is isomorphic to $P_{a}\{m\}$ for some $a \in B^{n}$ and $m \in \mathbb{Z}$.

Proof More generally, let $R$ be a $\mathbb{Z}_{+}$-graded ring, $R=\oplus_{i \geq 0} R_{i}$ such that $R_{0}$ is isomorphic to a finite direct sum $\mathbb{Z}^{\oplus j}$ of rings $\mathbb{Z}$. Our ring $H^{n}$ is of this form. Let $1_{i}, 1 \leq i \leq j$ be the minimal idempotents of $R$. We have:

Lemma 1 An idecomposable graded projective left $R$-module is isomorphic to $R 1_{i}\{m\}$ for some $i$ and $m$.

Sketch of proof If $M$ is a graded $R$-module, $M^{\prime} \stackrel{\text { def }}{=} M / R_{>0} M$ is a graded $\mathbb{Z}^{\oplus j}$-module and decomposes into direct sum of abelian groups,

$$
M^{\prime}=\underset{1 \leq i \leq j, k \in \mathbb{Z}}{\oplus} M^{\prime}{ }_{i, m},
$$

where $M_{i, m}^{\prime}$ is the degree $m$ direct summand for the idempotent $1_{i}$.

If $M$ is projective, $M \oplus N \cong F$, where $F$ is a free module, a direct sum of copies of $R$, with shifts in the grading. This induces an isomorphism of graded $R_{0}$-modules $M^{\prime} \oplus N^{\prime} \cong F^{\prime}$. We can find $i$ and $m$ such that $M^{\prime}{ }_{i, m} \neq$ 0 . Then there is a surjection of abelian groups $M^{\prime}{ }_{i, m} \rightarrow \mathbb{Z}$. It extends to a surjective map $M^{\prime}{ }_{i, m} \oplus N_{i, m}^{\prime} \cong F_{i, m}^{\prime} \rightarrow \mathbb{Z}$. From this and an isomorphism $F \cong$ $\underset{i, m}{\oplus} F_{i, m}^{\prime} \otimes R 1_{i}\{m\}$ we obtain an $R$-module homomorphism $F \rightarrow R 1_{i}\{m\}$. This homomorphism restricts to a surjective homomorphism $M \rightarrow R 1_{i}\{m\}$ (this homomorphism is surjective in degree $m$, therefore surjective since $R 1_{i}\{m\}$ is generated by $\mathbb{Z}$ in degree $m$ ).

Remark This proposition classified all graded projective $H^{n}$-modules. If we forget the grading, it is still true that all projective $H^{n}$-modules are standard: any indecomposable projective $H^{n}$-module is isomorphic to $P_{a}$, for some $a$. More generally, if $R$ is as before and, in addition, finitely-generated as an abelian group, then any indecomposable projective $R$-module is isomorphic to $R 1_{i}$ for some $i$.

We denote by $H_{P}^{n}$-mod the full subcategory of $H^{n}$-mod that consists of projective modules.

Denote by ${ }_{a} P$ the right $H^{n}$-module $\underset{b \in B^{n}}{\oplus} a\left(H^{n}\right)_{b}$. This is an indecomposable right projective $H^{n}$-module. 


\subsection{Bimodules and functors}

\section{a Sweet bimodules}

Definition 1 Given rings $C_{1}, C_{2}$, a $\left(C_{1}, C_{2}\right)$-bimodule $N$ is called sweet if it is finitely-generated and projective as a left $C_{1}$-module and as a right $C_{2}$-module.

The tensor product over $C_{1}$ with a $\left(C_{1}, C_{2}\right)$-bimodule $N$ is a functor from the category of right $C_{1}$-modules to the category of right $C_{2}$-modules. The tensor product over $C_{2}$ with $N$ is a functor from the category of left $C_{2}$-modules to the category of left $C_{1}$-modules. If $N$ is sweet, these functors are exact and take projective modules to projective modules. The tensor product $N \otimes_{C_{2}} M$ of a sweet $\left(C_{1}, C_{2}\right)$-bimodule $N$ with a sweet $\left(C_{2}, C_{3}\right)$-bimodule $M$ is a sweet $\left(C_{1}, C_{3}\right)$-bimodule.

To simplify notations, an $\left(H^{m}, H^{n}\right)$-bimodule will also be called an $(m, n)$ bimodule. The functor of tensoring with a sweet $(m, n)$-bimodule preserves the subcategory $H_{P}^{n}$-mod of $H^{n}$-mod that consists of projective modules and their homomorphisms.

\section{b Categories of complexes}

Given an additive category $\mathcal{S}$, we will denote by $\mathcal{K}(\mathcal{S})$ the category of bounded complexes in $\mathcal{S}$ up to chain homotopies. Objects of $\mathcal{K}(\mathcal{S})$ are bounded complexes of objects in $\mathcal{S}$. The abelian group of morphisms from an object $M$ of $\mathcal{K}(\mathcal{S})$ to $N$ is the quotient of the abelian group $\oplus_{i \in \mathbb{Z}} \operatorname{Hom}_{\mathcal{S}}\left(M^{i}, N^{i}\right)$ by the nullhomotopic morphisms, i.e. those that can be presented as $h d_{M}+d_{N} h$ for some $h=\left\{h_{i}\right\}, h_{i} \in \operatorname{Hom}_{\mathcal{S}}\left(M^{i}, N^{i-1}\right)$. We sometimes refer to $\mathcal{K}(\mathcal{S})$ as the homotopy category of $\mathcal{S}$.

For $n \in \mathbb{Z}$ denote by $[n]$ the automorphism of $\mathcal{K}(\mathcal{S})$ that is defined on objects by $N[n]^{i}=N^{i+n}, d[n]^{i}=(-1)^{n} d^{i+n}$ and continued to morphisms in the obvious way.

A complex homotopic to the zero complex is called contractible. A complex

$$
\ldots \longrightarrow 0 \longrightarrow T \stackrel{\mathrm{Id}}{\longrightarrow} T \longrightarrow 0 \ldots, \quad T \in \mathrm{Ob}(\mathcal{S}),
$$

is contractible. If $\mathcal{S}$ is an abelian category (or, more generally, an additive category with split idempotents) then any bounded contractible complex is isomorphic to the direct sum of complexes of type (18).

The cone of a morphism $f: M \rightarrow N$ of complexes is a complex $C(f)$ with

$$
C(f)^{i}=M[1]^{i} \oplus N^{i}, \quad d_{C(f)}\left(m^{i+1}, n^{i}\right)=\left(-d_{M} m^{i+1}, f\left(m^{i+1}\right)+d_{N} n^{i}\right) .
$$


The cone of the identity map from a complex to itself is contractible.

If the category $\mathcal{S}$ is monoidal, so is $\mathcal{K}(\mathcal{S})$, with the tensor product

$$
\begin{aligned}
& (M \otimes N)^{i}=\underset{j}{\oplus} M^{j} \otimes N^{i-j}, \\
& d(m \otimes n)=d m \otimes n+(-1)^{j} m \otimes d n, \quad m \in M^{j}, n \in N .
\end{aligned}
$$

We denote the category $\mathcal{K}\left(H_{P}^{n}\right.$-mod $)$ by $\mathcal{K}_{P}^{n}$. Its objects are bounded complexes of finitely-generated graded projective left $H^{n}$-modules (with gradingpreserving differentials). Denote the category $\mathcal{K}\left(H^{n}\right.$-mod) by $\mathcal{K}^{n}$.

Tensoring an object of $\mathcal{K}_{P}^{n}$ with a sweet $(m, n)$-bimodule gets us an object of $\mathcal{K}_{P}^{m}$. More generally, tensoring with a complex $N$ of sweet $(m, n)$-bimodules is a functor from $\mathcal{K}_{P}^{n}$ to $\mathcal{K}_{P}^{m}$, and from $\mathcal{K}^{n}$ to $\mathcal{K}^{m}$.

\subsection{Plane diagrams and bimodules}

Let $a \in \widehat{B}_{n}^{m}$. Define an $(m, n)$-bimodule $\mathcal{F}(a)$ by

$$
\mathcal{F}(a)=\bigoplus_{b, c} c \mathcal{F}(a)_{b},
$$

where $b$ ranges over elements of $B^{n}$ and $c$ over elements of $B^{m}$ and

$$
{ }_{c} \mathcal{F}(a)_{b} \stackrel{\text { def }}{=} \mathcal{F}(W(c) a b)\{n\}
$$

The left action $H^{m} \times \mathcal{F}(a) \rightarrow \mathcal{F}(a)$ comes from maps

$$
{ }_{d}\left(H^{m}\right)_{c} \times{ }_{c} \mathcal{F}(a)_{b} \longrightarrow{ }_{d} \mathcal{F}(a)_{b}
$$

induced by the cobordism from $W(d) c W(c) a b$ to $W(d) a b$ which is the composition of the identity cobordisms $W(d) \rightarrow W(d), a b \rightarrow a b$ and the standard cobordism $S(c): c W(c) \rightarrow$ Vert $_{2 m}$, defined in Section 2.4.

Similarly, the right action $\mathcal{F}(a) \times H^{n} \rightarrow \mathcal{F}(a)$ is defined by maps

$$
{ }_{d} \mathcal{F}(a)_{c} \times{ }_{c} H_{b}^{m} \longrightarrow{ }_{d} \mathcal{F}(a)_{b}
$$

induced by the cobordism from $W(d) a c W(c) b$ to $W(d) a b$ obtained as the composition of the identity cobordisms of $W(d) a$ and $b$ and the standard cobordism $c W(c) \rightarrow$ Vert $_{2 m}$.

Let us illustrate this definition with some examples. If $n=m$ and $a$ is isotopic to the configuration Vert $2 n$ of $2 n$ vertical lines, then $\mathcal{F}(a)$ is isomorphic to $H^{n}$, with the natural $(n, n)$-bimodule structure of $H^{n}$. In fact, the shift by $\{n\}$ in the formula (20) was chosen to make $\mathcal{F}\left(\right.$ Vert $\left._{2 n}\right)$ isomorphic to $H^{n}$. 
If $a \in B^{n}$ then $\mathcal{F}(a)$ is isomorphic to the left $H^{n}$-module $P_{a}\{-n\}$ and $\mathcal{F}(W(a))$ to the right $H^{n}$-module ${ }_{a} P$.

If $b \in \widehat{B}_{n}^{m}$ is obtained by adding a circle to $a$, then

$$
\mathcal{F}(b) \cong \mathcal{F}(a) \otimes \mathcal{A} \cong \mathcal{F}(a)\{1\} \oplus \mathcal{F}(a)\{-1\} .
$$

Our definition of $\mathcal{F}(a)$ implies:

Lemma 2 Let $a \in \widehat{B}_{n}^{m}$. The bimodule $\mathcal{F}(a)$ is isomorphic, as a left $H^{m}$ module, to the direct sum $\oplus_{b \in B^{n}} \mathcal{F}(a b)\{n\}$ and, as a right $H^{n}$-module, to the direct sum $\oplus_{b \in B^{m}} \mathcal{F}(W(b) a)$.

Proposition 3 Let $a \in \widehat{B}_{n}^{m}$. The bimodule $\mathcal{F}(a)$ is a sweet $(m, n)$-bimodule.

Proof We must check that $\mathcal{F}(a)$ is projective as a left $H^{m}$-module and as a right $H^{n}$-module. By the preceeding lemma, to prove that $\mathcal{F}(a)$ is projective as a left $H^{m}$-module, it suffices to check that $\mathcal{F}(a b)$ is left $H^{m}$-projective for any $b \in B^{m}$. The diagram $a b$ contains some number (say, $k$ ) of closed circles. After removing these circles from $a b$, we get a diagram isotopic to a diagram in $B^{m}$. Denote the latter diagram by $c$. Then the left $H^{m}$-modules $\mathcal{F}(a b)$ and $P_{c} \otimes \mathcal{A}^{\otimes k}$ are isomorpic and, since $P_{c}$ is projective, $\mathcal{F}(a b)$ and $\mathcal{F}(a)$ are projective as well. Similarly, $\mathcal{F}(a)$ is right $H^{n}$-projective.

Proposition 4 An isotopy between $a, b \in \widehat{B}_{n}^{m}$ induces an isomorphism of bimodules $\mathcal{F}(a) \cong \mathcal{F}(b)$. Two isotopies between $a$ and $b$ induce equal isomorphisms iff the bijections from circle components of a to circle components of $b$ induced by the two isotopies coincide.

Proof An isotopy from $a$ to $b$ induces an isotopy from $W(e) a c$ to $W(e) b c$ for all $e \in B^{m}$ and $c \in B^{n}$. These isotopies induce isomorphisms of graded abelian groups $\mathcal{F}(W(e) a c) \cong \mathcal{F}(W(e) b c)$. Summing over all $e$ and $c$ we obtain a bimodule isomorphism $\mathcal{F}(a) \cong \mathcal{F}(b)$.

An isotopy of flat tangles is a special case of an admissible cobordism (see section 2.2). An admissible cobordism also induces a bimodule map:

Proposition 5 Let $a, b \in \widehat{B}_{n}^{m}$ and $S$ an admissible surface with $\partial_{0} S=a$ and $\partial_{1} S=b$. Then $S$ defines a homomorphism of $(m, n)$-bimodules

$$
\mathcal{F}(S): \mathcal{F}(a) \rightarrow \mathcal{F}(b)\{\chi(S)-n-m\},
$$

where $\chi(S)$ is the Euler characteristic of $S$ (the shift is there to make the map grading-preserving). 
Proof We have $\mathcal{F}(a)=\underset{c, e}{\oplus} \mathcal{F}(W(e) a c)\{n\}$ and $\mathcal{F}(b)=\underset{b, c}{\oplus} \mathcal{F}(W(e) b c)\{n\}$ where the sum is over $c \in B^{n}$ and $e \in B^{m}$. The surface $S$ induces a cobordism from $W(e) a c$ to $W(e) b c$ defined as the composition of the identity cobordism from $c$ to $c$, cobordism $S$ from $a$ bo $b$ and the identity cobordism from $W(e)$ to $W(e)$. This cobordism is represented by a surface $S^{\prime}$ that contains $S$ as a closed submanifold. $S^{\prime}$ induces a map of graded abelian groups $\mathcal{F}(W(e) a c) \rightarrow$ $\mathcal{F}(W(e) b c)$. Summing over all $c$ and $e$ we get a map $\mathcal{F}(a) \rightarrow \mathcal{F}(b)$ which is, obviously, a bimodule map. According to Section 2.1 this map has degree $-\chi\left(S^{\prime}\right)=n+m-\chi(S)$ and, after a shift, we get a grading-preserving bimodule map $\mathcal{F}(a) \rightarrow \mathcal{F}(b)\{\chi(S)-n-m\}$ which we will denote $\mathcal{F}(S)$.

Proposition 6 Isotopic admissible surfaces induce equal bimodule maps.

Proof Suppose that admissible surfaces $S_{1}$ and $S_{2}$ are isotopic. This isotopy keeps the boundary of $S_{1}$ and $S_{2}$ fixed, so that $\partial_{0} S_{1}=\partial_{0} S_{2}, \partial_{1} S_{1}=\partial_{1} S_{2}$, and there are canonical bimodule isomorphisms $\mathcal{F}\left(\partial_{0} S_{1}\right) \cong \mathcal{F}\left(\partial_{0} S_{2}\right)$ and $\mathcal{F}\left(\partial_{1} S_{1}\right) \cong$ $\mathcal{F}\left(\partial_{1} S_{2}\right)$. The proposition says that the diagram below is commutative

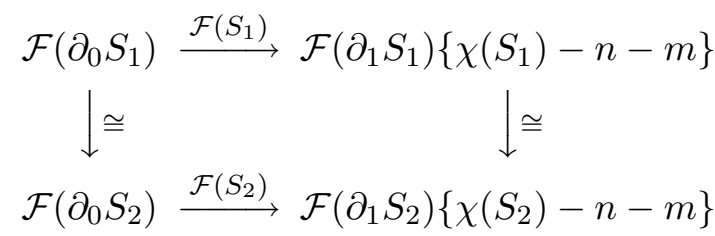

which easily follows from our definition of the bimodule map associated to a surface and the invariance of $\mathcal{F}$ under isotopies of slim surfaces.

Proposition 7 Let $a, b, c \in \widehat{B}_{n}^{m}$ and admissible surfaces $S_{1}$ and $S_{2}$ define cobordisms from $a$ to $b$ and from $b$ to $c$, respectively. Then $\mathcal{F}\left(S_{2}\right) \mathcal{F}\left(S_{1}\right)=$ $\mathcal{F}\left(S_{2} \circ S_{1}\right)$ where $S_{2} \circ S_{1}$ is the cobordism from a to $c$ obtained by composing surfaces $S_{1}$ and $S_{2}$.

This proposition says that the bimodule map associated to the composition of surfaces $S_{1}$ and $S_{2}$ is equal to the composition of bimodule maps associated to $S_{1}$ and $S_{2}$. That follows immediately from the functoriality of $\mathcal{F}$.

Theorem 1 For $a \in \widehat{B}_{n}^{m}$ and $b \in \widehat{B}_{m}^{k}$ there is a canonical isomorphism of $(k, n)$-bimodules

$$
\mathcal{F}(b a) \cong \mathcal{F}(b) \otimes_{H^{m}} \mathcal{F}(a) .
$$


Proof Define $\psi: \mathcal{F}(b) \otimes_{\mathbb{Z}} \mathcal{F}(a) \rightarrow \mathcal{F}(b a)$ via a commutative diagram

$$
\begin{array}{ccc}
\mathcal{F}(b) \otimes_{\mathbb{Z}} \mathcal{F}(a) & \psi & \mathcal{F}(b a) \\
\downarrow \cong & \mid \cong \\
\underset{c, d_{1}, d_{2}, e}{\oplus} \mathcal{F}\left(W(e) b d_{1}\right) \otimes \mathcal{F}\left(W\left(d_{2}\right) a c\right)\{n+m\} & \stackrel{\phi}{\longrightarrow} \underset{c, e}{\oplus \mathcal{F}(W(e) b a c)\{n\}}
\end{array}
$$

where the bottom map $\phi$ is zero if $d_{1} \neq d_{2}$ and otherwise (when $d_{1}=d_{2}$ ) induced by the minimal cobordism from $d_{1} W\left(d_{1}\right)$ to Vert $_{2 m}$.

The resulting map $\psi$ is, first of all, a $(k, n)$-bimodule map, where the left $H^{k}$ action on $\mathcal{F}(b) \otimes_{\mathbb{Z}} \mathcal{F}(a)$ comes from left $H^{k}$ action on $\mathcal{F}(b)$ and the right $H^{n}$ action from right action on $\mathcal{F}(a)$.

Moreover, $\psi$ factors through $\mathcal{F}(b) \otimes_{H^{m}} \mathcal{F}(a)$. To check this, let $m_{1} \in e_{e} \mathcal{F}(b)_{d_{1}}$, $x \in d_{1}\left(H^{m}\right)_{d_{2}}$, and $m_{2} \in d_{2} \mathcal{F}(a c)$. We claim that

$$
\psi\left(m_{1} x \otimes m_{2}\right)=\psi\left(m_{1} \otimes x m_{2}\right)
$$

The left and right hand sides of this equality can be described geometrically by two cobordisms between $W(e) b d_{1} W\left(d_{1}\right) d_{2} W\left(d_{2}\right) a c$ and $W(e) b a c$. Both cobordisms are compositions of minimal cobordisms on $d_{1} W\left(d_{1}\right)$ and $d_{2} W\left(d_{2}\right)$ and the identity cobordisms in the rest of the product. Relation (21) follows and so, indeed, $\psi$ factors through the map $\mathcal{F}(b) \otimes_{H^{m}} \mathcal{F}(a) \longrightarrow \mathcal{F}(b a)$ which we denote by $\psi^{\prime}$. The latter map is a $(k, n)$-bimodule map, since $\psi$ is. Therefore, the theorem will follow if we prove that $\psi^{\prime}$ is a bijective grading-preserving map of graded abelian groups.

$\psi^{\prime}$ is a direct sum of maps

$$
{ }_{e} \psi_{c}^{\prime}: e^{\mathcal{F}(b) \otimes_{H^{m}} \mathcal{F}(a)_{c} \longrightarrow e^{\mathcal{F}}(b a)_{c}}
$$

where $e$ and $c$ vary over elements of $B^{k}$ and $B^{n}$, respectively, and

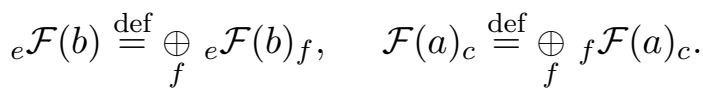

We have canonical isomorphisms of right $H^{m}$-modules $e_{e} \mathcal{F}(b) \cong \mathcal{F}(W(e) b)$, left $H^{m}$-modules $\mathcal{F}(a)_{c} \cong \mathcal{F}(a c)\{n\}$ and graded abelian groups $e_{e} \mathcal{F}(b a)_{c} \cong$ $\mathcal{F}(W(e) b a c)\{n\}$. We are thus reduced to establishing isomorphisms

$$
\mathcal{F}(W(e) b) \otimes_{H^{m}} \mathcal{F}(a c) \cong \mathcal{F}(W(e) b a c)
$$

of graded abelian groups.

Notice that $W(e) b$ is an element of $\widehat{B}_{m}^{0}$ and $a c$ an element of $\widehat{B}_{0}^{m}$. There are unique $x \in B^{m}$ and $y \in B^{n}$ such that $W(y)$ is isotopic to $W(e) b$ with all its 
circle component removed and $W(x)$ isotopic to $a c$ with all its circle component removed. Assuming that $W(e) b$ have $j_{1}$ and $a c$ have $j_{2}$ circle components, there are natural left/right $H^{m}$-module isomorphisms $\mathcal{F}(W(e) b) \cong \mathcal{A}^{\otimes j_{1}} \otimes$ $\mathcal{F}(W(y))$ and $\mathcal{F}(a c) \cong \mathcal{F}(x) \otimes \mathcal{A}^{\otimes j_{2}}$. Moreover, $\mathcal{F}(W(e) b a c) \cong \mathcal{F}(W(y) x) \otimes$ $\mathcal{A}^{\otimes j_{1}+j_{2}}$ and hence it suffices to prove the isomorphism

$$
\mathcal{F}(W(y)) \otimes_{H^{m}} \mathcal{F}(x) \cong \mathcal{F}(W(y) x)
$$

for $x, y \in B^{m}$. Notice that the right $H^{m}$-module $\mathcal{F}(W(y))$ is isomorphic to the right projective module ${ }_{y} P$, the left $H^{m}$-module $\mathcal{F}(x)$ is isomorphic to the left projective module $P_{x}\{-n\}$, and $\mathcal{F}(W(y) x)$ is isomorphic to ${ }_{y}\left(H^{m}\right)_{x}\{-n\}$. The desired formula $(2.7)$ thus transforms into ${ }_{y} P \otimes_{H^{m}} P_{x}={ }_{y}\left(H^{m}\right)_{x}$, which in turn follows from $H^{m} \otimes_{H^{m}} H^{m}=H^{m}$, by multiplying the latter by minimal idempotents $1_{x}$ and $1_{y}$ on the left and right respectively.

Proposition 8 The bimodule $\mathcal{F}(a)$ is indecomposable if $a \in B_{n}^{m}$. Bimodules $\mathcal{F}(a)$ and $\mathcal{F}(b)$, for $a, b \in B_{n}^{m}$ are isomorphic if and only if $a=b$.

We leave the proof to the reader. An equivalent form of the proposition is that

- $\mathcal{F}(a)$, for $a \in \widehat{B}_{n}^{m}$, is indecomposable if and only if $a$ does not contain circles;

- bimodules $\mathcal{F}(a)$ and $\mathcal{F}(b)$, for $a, b \in \widehat{B}_{n}^{m}$ are isomorphic if and only if $a$ and $b$ contain the same number of circles and the flat tangles obtained from $a$ and $b$ by removing all circles are isotopic.

\subsection{The category of geometric bimodules}

An $(m, n)$-bimodule is called geometric if it is isomorphic to a finite direct sum of bimodules $\mathcal{F}(a)$, possibly with shifts in the grading, for $a \in B_{n}^{m}$ (equivalently, for $\left.a \in \widehat{B}_{n}^{m}\right)$.

Notice that any geometric bimodule is sweet and that the tensor product of a geometric $(k, m)$-bimodule and a geometric $(m, n)$-bimodule is a geometric $(k, n)$-bimodule.

Let $\mathcal{S}_{n}^{m}$ be the category with objects-geometric $(m, n)$-bimodules and morphisms-bimodule homomorphisms (grading preserving, of course). The category $\mathcal{S}_{n}^{m}$ is additive. $\mathcal{S}_{0}^{m}$ is equivalent to $H_{P}^{m}$-mod, the category of finitely-generated projective $H^{m}$-modules. 
Tensor products of modules and bimodules can be viewed as bifunctors

$$
\begin{aligned}
\mathcal{S}_{m}^{k} \times \mathcal{S}_{n}^{m} & \longrightarrow \mathcal{S}_{n}^{k}, \\
\mathcal{S}_{n}^{m} \times H^{n} \text {-mod } & \longrightarrow H^{m} \text {-mod }, \\
\mathcal{S}_{n}^{m} \times H_{P}^{n} \text {-mod } & \longrightarrow H_{P}^{m} \text {-mod } .
\end{aligned}
$$

Let $\mathcal{K}_{n}^{m} \stackrel{\text { def }}{=} \mathcal{K}\left(\mathcal{S}_{n}^{m}\right)$ be the category of bounded complexes of objects of $\mathcal{S}_{n}^{m}$ up to chain homotopies. Tensor products of complexes give rise to bifunctors

$$
\begin{aligned}
\mathcal{K}_{m}^{k} \times \mathcal{K}_{n}^{m} & \longrightarrow \mathcal{K}_{n}^{k}, \\
\mathcal{K}_{n}^{m} \times \mathcal{K}^{n} & \longrightarrow \mathcal{K}^{m}, \\
\mathcal{K}_{n}^{m} \times \mathcal{K}_{P}^{n} & \longrightarrow \mathcal{K}_{P}^{m} .
\end{aligned}
$$

The category $\mathcal{K}_{0}^{m}$ is equivalent to $\mathcal{K}_{P}^{m}$.

\subsection{A 2-functor}

The results of Section 2.7 say that $\mathcal{F}$ is a 2-functor from the 2-category of surfaces with corners embedded in $\mathbb{R}^{3}$ to the 2-category of geometric $H$-bimodules and bimodule maps. In more details, let $\mathbb{G} \mathbb{B}$ be the 2-category with nonnegative integers as objects, geometric $(m, n)$-bimodules as 1-morphisms from $n$ to $m$, and bimodule homomorphisms as 2-morphisms. 1-morphisms from $n$ to $m$ and from $m$ to $k$ are composed by tensoring the bimodules over $H^{m}$. We call $\mathbb{G B}$ the 2-category of geometric $H$-bimodules. Observations from Section 2.7 summarize into:

Proposition $9 \mathcal{F}$ is a 2-functor from the Euler-Temperley-Lieb 2-category $\mathbb{E} \mathbb{L}$ to the 2-category $\mathbb{G B}$ of geometric $H$-bimodules.

Note that the objects of both 2-categories are nonnegative integers, and $\mathcal{F}$ is the identity on objects. It takes a 1-morphism $(a, j)$ of $\mathbb{E} \mathbb{T L}$ to the bimodule $\mathcal{F}(a)\{j\}$. We introduced $\mathbb{E} \mathbb{T L}$, a "central extension" of $\mathbb{T L}$, to make bimodule homomorphisms $\mathcal{F}(S)$ grading-preserving.

\section{Tangles and complexes of bimodules}

\subsection{Category of tangles}

We will only consider tangles with even number of top endpoints (notice that in any tangle the numbers of top and bottom endpoints have the same parity). 
An unoriented $(m, n)$-tangle $L$ is a proper, smooth embedding $\psi$ of $n+m$ arcs and a finite number of circles into $\mathbb{R}^{2} \times[0,1]$ such that:

(i) The boundary points of arcs map bijectively to the $2(n+m)$ points

$$
\{1,2, \ldots, 2 n\} \times\{0\} \times\{0\},\{1,2, \ldots, 2 m\} \times\{0\} \times\{1\} .
$$

The first $2 n$ points lie in $\mathbb{R}^{2} \times\{0\}$, the other $2 m$ in $\mathbb{R}^{2} \times\{1\}$.

(ii) Near the endpoints, the arcs are perpendicular to the boundary planes.

We impose (i) and (ii) to make tangles easy to concatenate. We distinguish between oriented and unoriented tangles. An oriented $(m, n)$-tangle comes with an orientation of each connected component.

Unoriented tangles constitute a category with objects-nonnegative integers, and morphisms-isotopy classes of $(m, n)$-tangles. The composition of morphisms is defined as the concatenation of tangles, in the same way as the composition of flat tangles was defined in Section 2.2.

Oriented tangles constitute a category, denoted $\mathcal{O} \mathcal{T} \mathcal{A N}$, with objects-even length sequences of \pm 1 , and morphisms-isotopy classes of oriented $(m, n)$ tangles. Our conventions are explained in Figure 12. An arc oriented upward near its boundary point marks this point with 1, a downward oriented arc with -1 .

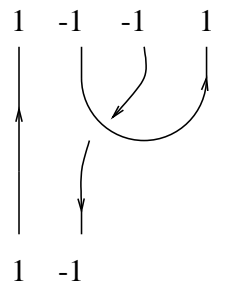

Figure 12: This oriented $(2,4)$-tangle is a morphism from $\{1,-1\}$ to $\{1,-1,-1,1\}$.

Any tangle is isotopic to a composition of elementary tangles, depicted in Figures 13-14 (to make our life easier, we will often draw piecewise-linear approximations of smooth tangles).

A plane diagram of a tangle is a generic projection of a tangle onto the $(x, z)$ plane (onto $\mathbb{R} \times[0,1]$ ). We call a projection generic if it has no triple intersections, tangencies and cusps. Two diagrams are called isotopic if they belong to a one-parameter family of generic projections.

Figure 15 explains the difference between isotopies of tangles and isotopies of plane diagrams. A deformation of a plane diagram is an isotopy if it does not change the combinatorial structure of the diagram. 

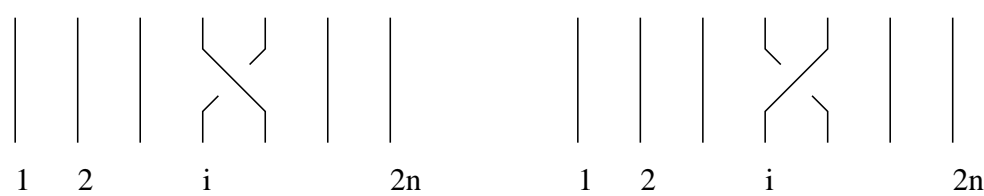

Figure 13: Tangles $\sigma_{i, 2 n}$ and $\sigma_{i, 2 n}^{-1}$

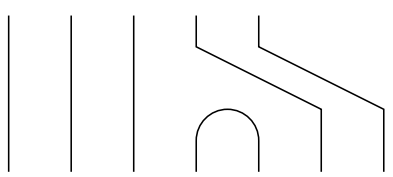

12

i

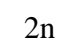

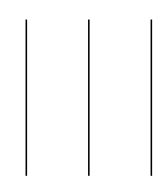

1

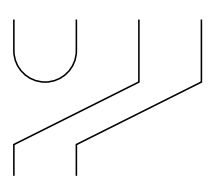

i 2 n

Figure 14: Tangles $\cap_{i, 2 n}$ and $\cup_{i, 2 n}$

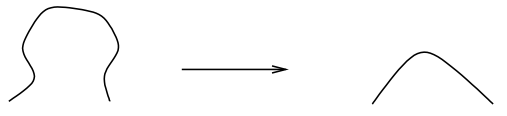

An isotopy of plane diagrams

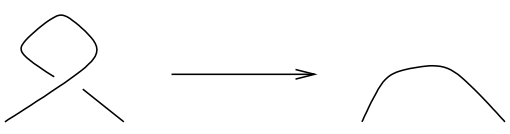

Not an isotopy of plane diagrams

Figure 15: Isotopies of plane diagrams explaned

Proposition 10 Two plane diagrams represent isotopic tangles if and only if these diagrams can be connected by a chain of diagram isotopies and Reidemeister moves, depicted in Figures 16-19.

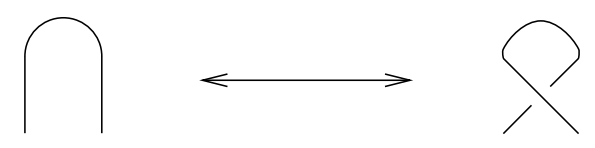

Figure 16: Addition/removal of a left-twisted curl

Algebraic 63 Geometric Topology, Volume 2 (2002) 


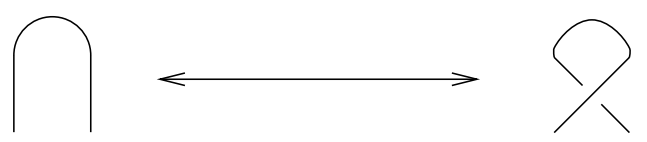

Figure 17: Addition/removal of a right-twisted curl

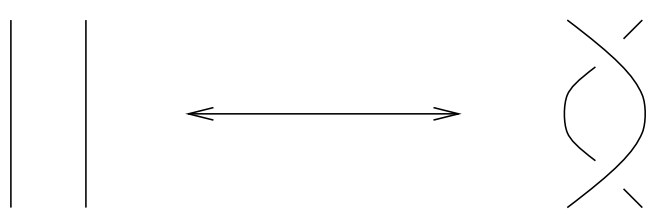

Figure 18: Tangency move
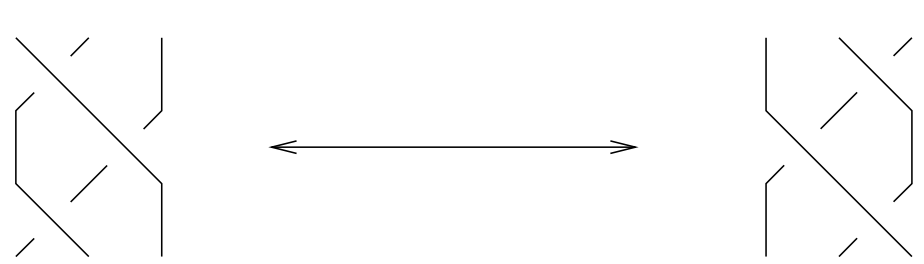

Figure 19: Triple point move

\subsection{Resolutions of plane diagrams and the Kauffman bracket}

Let $D$ be a diagram of an unoriented tangle $L$. A crossing of $D$ can be "resolved" in two possible ways, as in Figure 20.

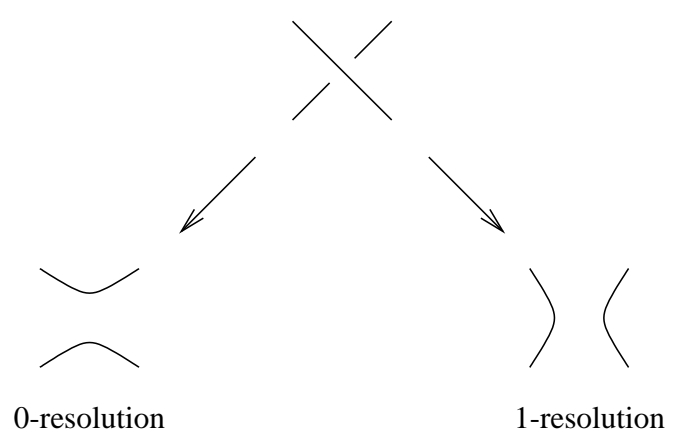

Figure 20: Two resolutions of a crossing

We call the resolution on the left 0 -resolution, the one on the right 1-resolution. A resolution of $D$ is a resolution of each double point of $D$. Thus, a resolution of a plane diagram is a flat tangle, and a morphism in the Temperley-Lieb category (see Section 2.2).

Algebraic $\mathcal{E} \mathcal{G}$ Geometric Topology, Volume 2 (2002) 
A diagram with $k$ crossings has $2^{k}$ resolutions. Define $\langle D\rangle$, the bracket of $D$, as the weighted sum

$$
\langle D\rangle=\sum_{s}\left(-q^{-1}\right)^{\#(s)} s,
$$

where $s$ varies over all resolutions of $D$ and $\#(s)$ is the number of 1-resolutions in $s$. We treat the sum as a morphism in the linear Temperley-Lieb category $\mathcal{L} \mathcal{T} \mathcal{L}$ (see Section 2.2)

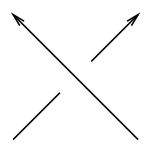

$x(D)$

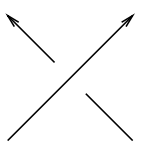

$y(D)$

Figure 21: Orientations of crossings

Let $D$ be a diagram of an oriented $(m, n)$-tangle $L$. Let $x(D)$ and $y(D)$ be the number of crossings of $D$ with local orientations as in Figure 21. To $D$ we associate the Kauffman bracket $K(D)$ by the formula

$$
K(D) \stackrel{\text { def }}{=}(-1)^{x(D)} q^{2 x(D)-y(D)}\langle D\rangle .
$$

Proposition $11 K(D)$ does not depend of the choice of a diagram $D$ of an oriented tangle $L$, and is an invariant of $L$.

We denote this invariant by $K(L)$ and call it the Kauffman bracket of the tangle $L$. It is an element of the free $\mathbb{Z}\left[q, q^{-1}\right]$-module generated by elements of $B_{n}^{m}$, and also a morphism from $n$ to $m$ in the linear Temperley-Lieb category.

Proposition 12 The Kauffman bracket is a functor from the category $\mathcal{O} \mathcal{T} \mathcal{N}$ of oriented tangles to the linear Temperley-Lieb category $\mathcal{L} \mathcal{T} \mathcal{L}$.

This functor takes an object of $\mathcal{O} \mathcal{T} \mathcal{A N}$ which is a sequence of \pm 1 of length $2 n$ to the object $n$ of $\mathcal{L} \mathcal{T} \mathcal{L}$. Notice also that $L$ is an oriented tangle while flat tangles are not oriented, according to our convention. When this functor is computed on an oriented tangle $L$ using its diagram $D$, the orientations of components of $L$ are discarded once we know $x(D)$ and $y(D)$.

The Kauffman bracket was discovered by Louis Kauffman [34], who also showed that after a simple change of variables the Kauffman bracket turns into the Jones polynomial. The usual formula in the literature for the Kauffman bracket 
appears somewhat more symmetric, due to the use of the square root of $q$. We steer clear of the square root at the cost of a normalization that employs 2 parameters, $x(D)$ and $y(D)$, rather than just one-the writhe. Moreover, in the literature the bracket of the closed circle is set to $-q-q^{-1}$, rather than our $q+q^{-1}$, so that our $q$ is the conventional $-q$.

\subsection{Commutative and anticommutative cubes}

This section is a repeat of [38, Sections 3.2-3.4], included here for completeness.

A commutative cube is a generalization of a commutative square. We assign an object of a category to each vertex of an $n$-dimensional cube and a morphism to each edge so that each 2-dimensional facet of the cube is a commutative diagram.

In details, let $I$ be a finite set, $|I|$ its cardinality, and $r(I)$ the set of all pairs $(T, a)$ where $T$ is a subset of $I$ and $a \in I \backslash T$. To simplify notation we will often denote a finite set $\{a, b, \ldots, d\}$ by $a b \ldots d$, the disjoint union $T_{1} \sqcup T_{2}$ of two sets by $T_{1} T_{2}$, so that $T a$, for instance, means $T \sqcup\{a\}$.

Definition 2 A commutative $I$-cube $V$ over a category $\mathcal{S}$ assigns an object $V(T)$ of $\mathcal{S}$ to each subset $T$ of $I$ and a morphism $V(T) \longrightarrow V(T a)$ to each $(T, a) \in r(I)$ such that the diagram

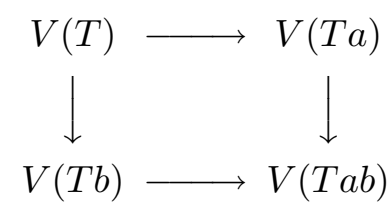

commutes for any triple $(T, a, b)$ where $T \subset I$, and $a, b \in I \backslash T, a \neq b$. The morphisms are called the structure maps of $V$.

We will call a commutative $I$-cube an $I$-cube or, sometimes, a cube without explicitly mentioning $I$.

If the category $\mathcal{S}$ is monoidal, commutative cubes over $\mathcal{S}$ admit internal and external tensor products. The internal product of two $I$-cubes $V$ and $W$ is an $I$-cube, denoted $V \otimes W$, with $(V \otimes W)(T)=V(T) \otimes W(T)$ and structure maps defined in the obvious way. The external tensor product of an $I_{1}$-cube $V$ and an $I_{2}$-cube $W$ is an $I_{1} I_{2}$-cube $V \otimes W$ with $(V \otimes W)\left(T_{1} T_{2}\right)=V\left(T_{1}\right) \otimes W\left(T_{2}\right)$, where $T_{i} \subset I_{i}$, and obviously defined structure maps. 
A skew-commutative $I$-cube over an additive category $\mathcal{S}$ is defined in the same way as a commutative $I$-cube, except that we require that for every square facet of the cube the associated diagram of objects and morphisms of $\mathcal{S}$ anticommutes.

Define a skew-commutative $I$-cube $E(I)$ over the category of abelian groups as follows. For a finite set $T$ denote by $o(T)$ the set of total orderings or elements of $T$. For $x, y \in o(T)$ let $p(x, y)$ be the parity function, $p(x, y)=0$ if $y$ can be obtained by from $x$ via an even number of transpositions of two elements in the ordering, otherwise, $p(x, y)=1$. To $T$ associate an abelian group $E(T)$ which is the quotient of the free abelian group generated by $x$ for all $x \in o(T)$ by relations $x=(-1)^{p(x, y)} y$ for all pairs $x, y \in o(T)$. Notice that $E(T)$ is isomorphic to $\mathbb{Z}$. For $a \notin T$ the map $o(T) \rightarrow o(T a)$ that takes $x \in o(T)$ to $a x \in o(T a)$ induces an isomorphism $E(T) \cong E(T a)$. Moreover, the diagram below anticommutes.

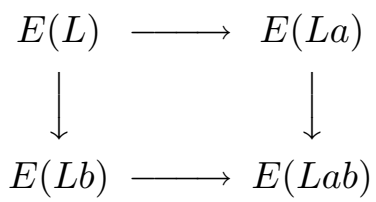

Denote by $E_{I}$ the skew-commutative $I$-cube with $E_{I}(T)=E(T)$ for $T \subset I$ and the above isomorphisms $E(T) \cong E(T a)$ as structure maps.

Note that in [38, Section 3.3] the structure maps of $E_{I}$ take $x$ to $x a=(-1)^{|x|} a x$, rather than to $a x$. We changed the definition to make Lemma 3 (see below) hold.

If $V$ is a commutative $I$-cube over an additive category $\mathcal{S}$, the internal tensor product $V \otimes E_{I}$ is a skew-commutative $I$-cube over $\mathcal{S}$. Essentially, the tensor product with $E_{I}$ adds minus signs to some structure maps of $V$, making each square anticommute. Since $E_{I}$ is defined in a rather invariant way, the minuses stay hidden, however.

To a skew-commutative $I$-cube $W$ over $\mathcal{S}$ we associate a complex $\bar{C}(W)$ of objects of $\mathcal{S}$ by

$$
\bar{C}^{i}(W)=\underset{T \subset I,|T|=i}{\oplus} W(T)
$$

and the differential $d$ is the sum of the structure maps of $W$. Skew-commutativity of square faces of $V$ ensures that $d^{2}=0$.

To a commutative $I$-cube $V$ over $\mathcal{S}$ we associate the complex $\bar{C}\left(V \otimes E_{I}\right)$ of objects of $\mathcal{S}$. 
Assume now that $\mathcal{S}$ is an additive monoidal category. Then the category of $\mathcal{S}$-complexes is also monoidal. Let $V_{1}, V_{2}$ be commutative $I_{1}, I_{2}$-cubes over $\mathcal{S}$. The following lemma says that there are two equivalent ways to produce a complex from this data: either take the complex associated to the external tensor product of $V_{1}$ and $V_{2}$, or take the tensor product of complexes associated to $V_{1}$ and $V_{2}$.

Lemma 3 Complexes $\bar{C}\left(\left(V_{1} \otimes V_{2}\right) \otimes E_{I_{1} I_{2}}\right)$ and $\bar{C}\left(V_{1} \otimes E_{I_{1}}\right) \otimes \bar{C}\left(V_{2} \otimes E_{I_{2}}\right)$ are isomorphic, via the map which sends $\left(t_{1} \otimes t_{2}\right) \otimes\left(x_{1} x_{2}\right)$, considered as an element of the first complex (where $t_{i} \in V_{i}\left(T_{i}\right)$ and $x_{i} \in o\left(T_{i}\right)$ for some $T_{i} \subset I_{i}$ ) to $\left(t_{1} \otimes x_{1}\right) \otimes\left(t_{2} \otimes x_{2}\right)$, considered as an element of the second complex.

Proof We just have to check that the above identification of terms in the two complexes is consistent with the differentials in the complexes. That follows from our definitions of $E_{I}$ and the differential in the tensor product (19). If $a$ is an element of $I_{2} \backslash T_{2}$, we have $a x_{1} x_{2}=(-1)^{\left|x_{1}\right|} x_{1} a x_{2}$, the same power of -1 as in the formula (19) for the differential of the tensor product.

\subsection{The complex associated to a tangle diagram}

Fix a plane diagram $D$ with $k$ crossings of an oriented $(m, n)$-tangle $L$. Let $I$ be the set of crossings of $D$. To $D$ we will associate an $I$-cube $V_{D}$ over the category of $(m, n)$-bimodules. This cube will not depend on the orientation of components of $L$.

$D$ admits $2^{k}$ resolutions (see Section 3.2), in bijection with subsets of $I$ : given $T \subset I$, take 1-resolution of each crossing that belongs to $T$, and 0-resolution of each crossing that doesn't. Denote by $D(T)$ the resolution associated to $T$. Each resolution of $D$ is a flat $(m, n)$-tangle and $\mathcal{F}(D(T))$ is an $(m, n)$-bimodule. We assign this bimodule, with the grading lowered by the cardinality of $T$, to the vertex of $V_{D}$ associated to $T$ :

$$
V_{D}(T) \stackrel{\text { def }}{=} \mathcal{F}(D(T))\{-|T|\} .
$$

To define the structure maps $V_{D}(T) \longrightarrow V_{D}(T a)$, for $a \in I \backslash T$, we notice that resolutions $D(T)$ and $D(T a)$ of $D$ differ only in a small neighbourhood $U$ of the crossing $a$ of $D$ (see Figure 22, the dashed circle is the boundary of $U$ ).

There is an admissible cobordism $S$ between $D(T)$ and $D(T a)$, unique up to isotopy, which is the identity cobordism outside $U \times[0,1]$, and the simplest cobordism between $U \cap D(T)$ and $U \cap D(T a)$ inside $U \times[0,1]$. This cobordism 

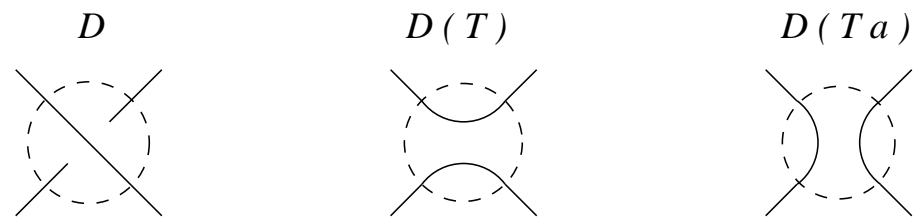

Figure 22: $D$ and the two resolutions in the neighbourhood of $a$

has one saddle point and no other other critical points relative to the height function $S \subset \mathbb{R}^{2} \times[0,1] \longrightarrow[0,1]$. For a more detailed description of $S$ we refer the reader to $[38$, Section 4.2].

$\mathcal{F}(S): \mathcal{F}(D(T)) \longrightarrow \mathcal{F}(D(T a))$ is a degree 1 homomorphism of $(m, n)$ bimodules, therefore, after shifts it becomes a grading-preserving bimodule homomorphism

$$
\mathcal{F}(S): \mathcal{F}(D(T))\{-|T|\} \longrightarrow \mathcal{F}(D(T a))\{-|T a|\},
$$

since $|T a|=|T|+1$. This is the homomorphism we assign to the oriented edge of the cube $V_{D}$ connecting vertices labeled by $T$ and $T a$. Functoriality of $\mathcal{F}$ implies that every square face of $V_{D}$ is commutative.

Tensoring $V_{D}$ with $E_{I}$, we get a skew-commutative $I$-cube $V_{D} \otimes E_{I}$. Denote by $\overline{\mathcal{F}}(D)$ the complex $\bar{C}\left(V_{D} \otimes E_{I}\right)$, and by $\mathcal{F}(D)$ the shifted complex

$$
\mathcal{F}(D) \stackrel{\text { def }}{=} \overline{\mathcal{F}}(D)[x(D)]\{2 x(D)-y(D)\} .
$$

\subsection{Main result}

Theorem 2 If $D_{1}, D_{2}$ are diagrams of an oriented $(m, n)$-tangle $L$, the complexes $\mathcal{F}\left(D_{1}\right)$ and $\mathcal{F}\left(D_{2}\right)$ of $(m, n)$-bimodules are chain homotopy equivalent.

The proof occupies Section 4.

It follows that the isomorphism class of $\mathcal{F}(D)$ in the category $\mathcal{K}_{n}^{m}$ does not depend on the choice of a diagram $D$ of an oriented tangle $L$, and is an invariant of $L$, denoted $\mathcal{F}(L)$.

\section{Proof of Theorem 2}

\subsection{Invariance under isotopies of plane diagrams}

An isotopy $\gamma$ between plane diagrams $D_{1}$ and $D_{2}$ induces a bijection $\gamma_{*}$ : $I_{1} \cong I_{2}$ between their sets of crossings. There is a canonical isotopy between 
resolutions $D_{1}\left(T_{1}\right)$ and $D_{2}\left(\gamma_{*} T_{1}\right)$, for any subset $T_{1}$ of $I_{1}$, giving rise to an isomorphism of bimodules $\mathcal{F}\left(D_{1}\left(T_{1}\right)\right) \cong \mathcal{F}\left(D_{2}\left(\gamma_{*} T_{1}\right)\right)$. These isomorphisms respect structure maps and provide us with an isomorphism of cubes $V_{D_{1}}$ and $V_{D_{2}}$. This isomorphism immediately leads to an isomorphism between complexes $\mathcal{F}\left(D_{1}\right)$ and $\mathcal{F}\left(D_{2}\right)$, since $x\left(D_{1}\right)=x\left(D_{2}\right)$ and $y\left(D_{1}\right)=y\left(D_{2}\right)$.

\subsection{Behaviour under composition of plane diagrams}

Proposition 13 Let $D_{2}, D_{1}$ be plane diagrams of unoriented $(k, m)$ - and $(m, n)$-tangles. There is a canonical isomorphism of complexes of $(k, n)$-bimodules

$$
\mathcal{F}\left(D_{2} D_{1}\right) \cong \mathcal{F}\left(D_{2}\right) \otimes_{H^{m}} \mathcal{F}\left(D_{1}\right)
$$

Proof Let $I_{i}$ be the set of crossings of $D_{i}$. Given subsets $T_{i} \subset I_{i}$, the resolution $D_{2} D_{1}\left(T_{2} T_{1}\right)$ of $D_{2} D_{1}$ is the composition of resolutions $D_{2}\left(T_{2}\right)$ and $D_{1}\left(T_{1}\right)$. Theorem 1 provides us with a canonical bimodule isomorphism

$$
\mathcal{F}\left(D_{2} D_{1}\left(T_{2} T_{1}\right)\right) \cong \mathcal{F}\left(D_{2}\left(T_{2}\right)\right) \otimes_{H^{m}} \mathcal{F}\left(D_{1}\left(T_{1}\right)\right) .
$$

If $a_{i} \in I_{i} \backslash T_{i}$, elementary cobordisms between $D_{i}\left(T_{i}\right)$ and $D_{i}\left(T_{i} a_{i}\right)$ induce bimodule maps $\mathcal{F}\left(D_{i}\left(T_{i}\right)\right) \longrightarrow \mathcal{F}\left(D_{i}\left(T_{i} a_{i}\right)\right)$ which make the diagram below commute (for $i=1$, similarly for $i=2$ )

$$
\begin{array}{ccc}
\mathcal{F}\left(D_{2} D_{1}\left(T_{2} T_{1}\right)\right) & \cong \mathcal{F}\left(D_{2}\left(T_{2}\right)\right) \otimes_{H^{m}} \mathcal{F}\left(D_{1}\left(T_{1}\right)\right) \\
\downarrow & & \downarrow \\
\mathcal{F}\left(D_{2} D_{1}\left(T_{2} T_{1} a_{1}\right)\right)\{-1\} & \cong \mathcal{F}\left(D_{2}\left(T_{2}\right)\right) \otimes_{H^{m}} & \mathcal{F}\left(D_{1}\left(T_{1} a_{1}\right)\right)\{-1\}
\end{array}
$$

Therefore, the external tensor product of commutative cubes $V_{D_{2}}$ and $V_{D_{1}}$ is canonically isomorphic to the commutative cube $V_{D_{2} D_{1}}$. Lemma 3 implies that there is a canonical isomorphism of complexes of bimodules

$$
\overline{\mathcal{F}}\left(D_{2} D_{1}\right) \cong \overline{\mathcal{F}}\left(D_{2}\right) \otimes_{H^{m}} \overline{\mathcal{F}}\left(D_{1}\right)
$$

Observing that $x(D)$ and $y(D)$ are additive under composition of diagrams,

$$
x\left(D_{2} D_{1}\right)=x\left(D_{2}\right)+x\left(D_{1}\right), \quad y\left(D_{2} D_{1}\right)=y\left(D_{2}\right)+y\left(D_{1}\right),
$$

we obtain isomorphism (26).

Any tangle can be written (in many ways, of course) as a composition of elementary tangles, depicted in figures 13,14 . Therefore, the complex $\mathcal{F}(D)$ is isomorphic to a tensor product of complexes associated to figure 13 and 14 diagrams of elementary tangles (referred to from now on as elementary diagrams). 
The invariance of $\mathcal{F}(D)$ under the Reidemeister moves can be checked locally. For instance, if $D_{1}$ and $D_{2}$ are related by a triple point move (figure 19), there are decompositions

$$
D_{1} \cong D^{\prime} \sigma_{i, 2 n} \sigma_{i+1,2 n} \sigma_{i, 2 n} D^{\prime \prime} \text { and } D_{2} \cong D^{\prime} \sigma_{i+1,2 n} \sigma_{i, 2 n} \sigma_{i+1,2 n} D^{\prime \prime},
$$

where $\cong$ above denotes isotopy of plane diagrams. These decompositions give rise to isomorphisms of complexes of bimodules

$$
\begin{aligned}
& \mathcal{F}\left(D_{1}\right) \cong \mathcal{F}\left(D^{\prime}\right) \otimes_{H^{n}} \mathcal{F}\left(\sigma_{i, 2 n} \sigma_{i+1,2 n} \sigma_{i, 2 n}\right) \otimes_{H^{n}} \mathcal{F}\left(D^{\prime \prime}\right), \\
& \mathcal{F}\left(D_{2}\right) \cong \mathcal{F}\left(D^{\prime}\right) \otimes_{H^{n}} \mathcal{F}\left(\sigma_{i+1,2 n} \sigma_{i, 2 n} \sigma_{i+1,2 n}\right) \otimes_{H^{n}} \mathcal{F}\left(D^{\prime \prime}\right) .
\end{aligned}
$$

Consequently, the invariance under triple point moves will follow once we construct a chain homotopy equivalence

$$
\mathcal{F}\left(\sigma_{i, 2 n} \sigma_{i+1,2 n} \sigma_{i, 2 n}\right) \cong \mathcal{F}\left(\sigma_{i+1,2 n} \sigma_{i, 2 n} \sigma_{i+1,2 n}\right) .
$$

Similar chain homotopy equivalences will imply invariance under the tangency and curl addition moves.

\subsection{Left-twisted curl}

Denote by $D$ the elementary tangle $\cap_{i, 2 n}$, arbitrarily oriented, by $D_{1}$ the diagram $D$ with a left-twisted curl added, and by $D_{2}$ the 0 -resolution of $D_{1}$, as depicted in Figure 23.

Note that $D$ is isotopic to the 1 -resolution of $D_{1}$. We want to construct an isomorphism in the category $\mathcal{K}_{n}^{n-1}$ between the bimodule $\mathcal{F}(D)$ and the complex of bimodules $\mathcal{F}\left(D_{1}\right)$, the latter isomorphic to the cone of a bimodule homomorphism $\mathcal{F}\left(D_{2}\right) \rightarrow \mathcal{F}(D)$ (in this informal discussion we will ignore shifts). Since, $\mathcal{F}\left(D_{2}\right) \cong \mathcal{F}(D) \otimes \mathcal{A}=\mathcal{F}(D) \oplus \mathcal{F}(D)$, and the homomorphism $\mathcal{F}\left(D_{2}\right) \rightarrow \mathcal{F}(D)$ is the identity when restricted to $\mathcal{F}(D)=\mathcal{F}(D) \otimes \mathbf{1} \subset \mathcal{F}\left(D_{2}\right)$, after taking the cohomology we'll be left with the remaining copy of $\mathcal{F}(D)$.

We will now beef up this intuitive sketch into a rigorous argument. Notice that $\mathcal{F}\left(D_{2}\right) \cong \mathcal{F}(D) \otimes \mathcal{A}$. Cobordisms in Figures 24, 25 and 26 induce (gradingpreserving) bimodule homomorphisms:

$$
\begin{array}{ccccc}
m_{0} & : & \mathcal{F}\left(D_{2}\right)\{1\} & \longrightarrow & \mathcal{F}(D) \\
\Delta_{0} & : & \mathcal{F}(D)\{1\} & \longrightarrow & \mathcal{F}\left(D_{2}\right) \\
\iota_{0} & : & \mathcal{F}(D) & \longrightarrow & \mathcal{F}\left(D_{2}\right)\{1\}
\end{array}
$$

These bimodule homomorphisms are similar to the structure maps $m, \Delta, \iota$ of the ring $\mathcal{A}$, hence the notation. Note that

$$
m_{0} \iota_{0}=\operatorname{Id}_{\mathcal{F}(D)} .
$$

Let $\jmath_{0}=\Delta_{0}-\iota_{0} m_{0} \Delta_{0}$. Then $m_{0} \jmath_{0}=0$. 


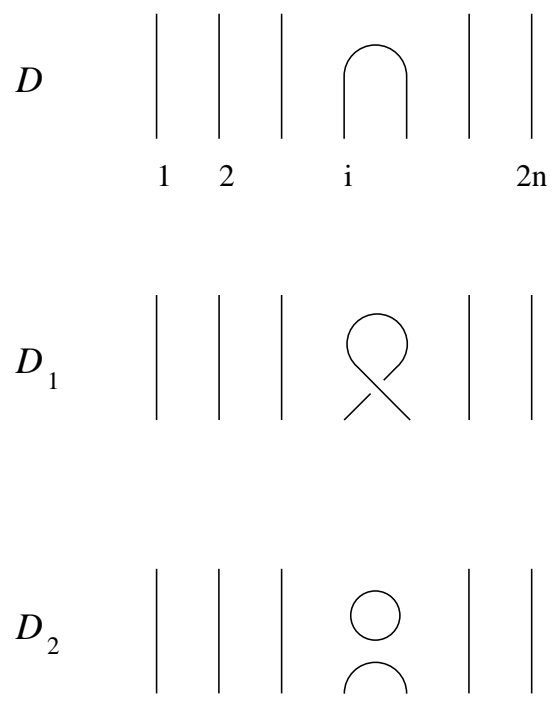

Figure 23: Left-twisted curl and its two resolutions
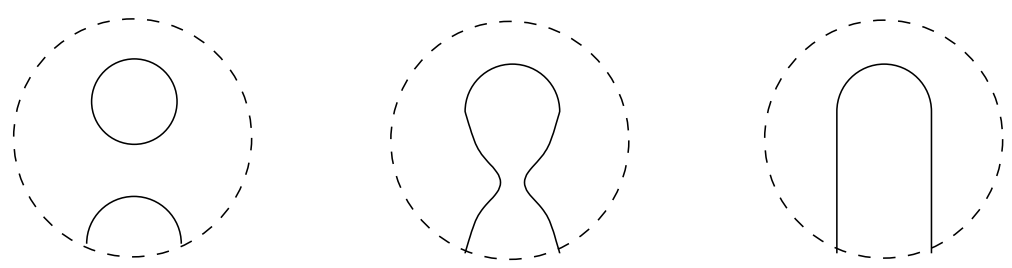

Figure 24: $m_{0}$ cobordism
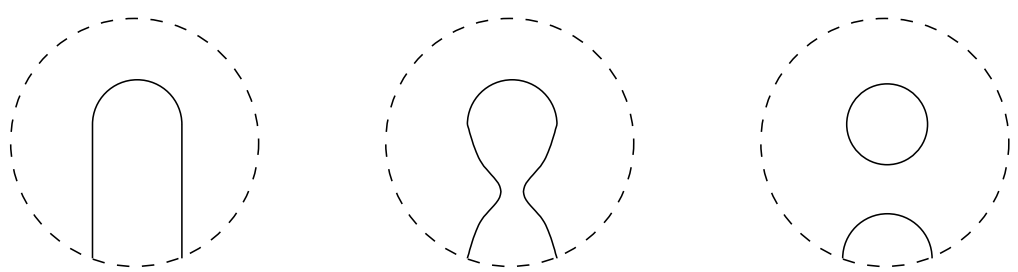

Figure 25: $\Delta_{0}$ cobordism

Proposition 14 Bimodule homomorphisms $\iota_{0}$ and $\jmath_{0}$ are injective and there is a direct sum decomposition of bimodules

$$
\mathcal{F}\left(D_{2}\right)=\iota_{0}(\mathcal{F}(D)\{-1\}) \oplus \jmath_{0}(\mathcal{F}(D)\{1\}) .
$$

The proof is straightforward.

Algebraic $\&$ Geometric $\mathcal{T}$ opology, Volume 2 (2002) 

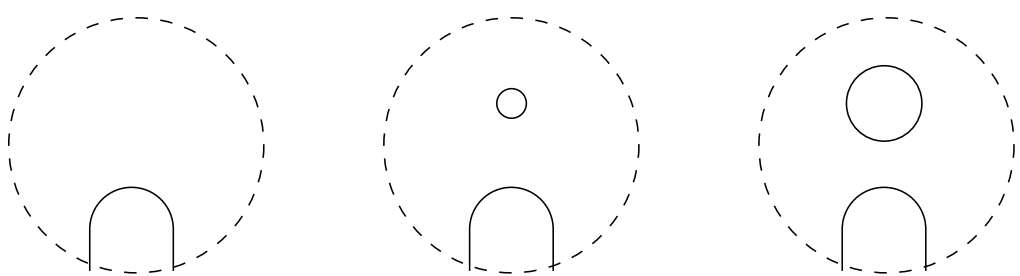

Figure 26: $\iota_{0}$ cobordism

The complex $\overline{\mathcal{F}}\left(D_{1}\right)$ is given by

$$
\ldots \longrightarrow 0 \longrightarrow \mathcal{F}\left(D_{2}\right) \stackrel{m_{0}}{\longrightarrow} \mathcal{F}(D)\{-1\} \longrightarrow 0 \ldots,
$$

which we can rewrite as

$$
0 \longrightarrow \iota_{0}(\mathcal{F}(D)\{-1\}) \oplus \jmath_{0}(\mathcal{F}(D)\{1\}) \stackrel{(\mathrm{id}, 0)}{\longrightarrow} \mathcal{F}(D)\{-1\} \longrightarrow 0 .
$$

This complex is isomorphic to the direct sum of

$$
0 \longrightarrow \mathcal{F}(D)\{1\} \longrightarrow 0
$$

and a contractible complex

$$
0 \longrightarrow \mathcal{F}(D)\{1\} \stackrel{\text { id }}{\longrightarrow} \mathcal{F}(D)\{1\} \longrightarrow 0,
$$

therefore, $\overline{\mathcal{F}}\left(D_{1}\right) \cong \mathcal{F}(D)\{1\}$ in the homotopy category $\mathcal{K}_{n}^{n-1}$.

Equalities $x\left(D_{1}\right)=x(D)=0$ and $y\left(D_{1}\right)=y(D)+1=1$, valid for any orientation of $D$, give an isomorphism $\mathcal{F}\left(D_{1}\right) \cong \mathcal{F}(D)$ in the homotopy category of complexes of bimodules.

\subsection{Right-twisted curl}

We let diagrams $D$ and $D_{2}$ be the ones in the previous subsection, and let $D_{1}$ be $D$ decorated by a right-twisted curl (figure 27). $D$ is isotopic to the 0-resolution of $D_{1}$ and $D_{2}$ to the 1-resolution of $D_{1}$.

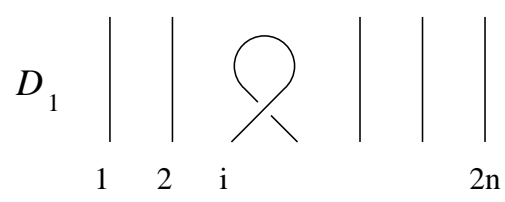

Figure 27: Right-twisted curl in the standard position 
We will use bimodule homomorphisms $m_{0}, \Delta_{0}, \iota_{0}$, defined in the previous subsection. In addition, introduce a bimodule homomorphism

$$
\epsilon_{0}: \mathcal{F}\left(D_{2}\right) \longrightarrow \mathcal{F}(D)\{1\}
$$

associated to the surface depicted in Figure 28.
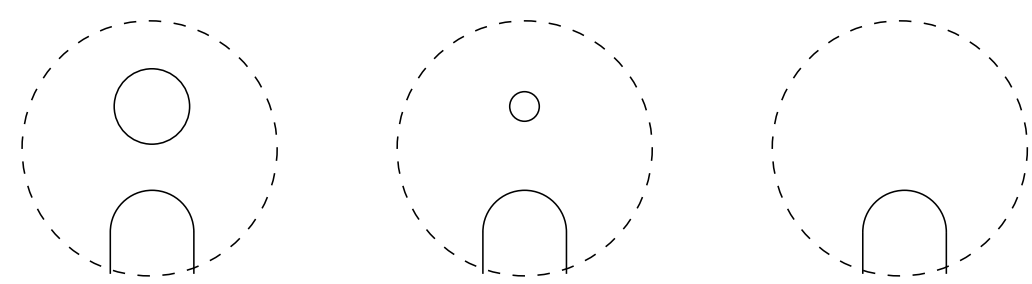

Figure 28: Cobordism for $\epsilon_{0}$

Proposition 15 There is a direct sum decomposition of bimodules

$$
\mathcal{F}\left(D_{2}\right)=\iota_{0}(\mathcal{F}(D)\{-1\}) \oplus \Delta_{0}(\mathcal{F}(D)\{1\}) .
$$

Denote by $\wp$ the bimodule homomorphism

$$
\wp=m_{0}-m_{0} \Delta_{0} \epsilon_{0}: \mathcal{F}\left(D_{2}\right)\{1\} \longrightarrow \mathcal{F}(D) .
$$

Lemma 4 We have equalities

$$
\begin{aligned}
\wp \Delta_{0} & =0 \\
\wp \iota_{0} & =\operatorname{Id}_{\mathcal{F}(D)}
\end{aligned}
$$

$\overline{\mathcal{F}}\left(D_{1}\right)$ is the cone of the bimodule homomorphism $\Delta_{0}: \mathcal{F}(D) \longrightarrow \mathcal{F}\left(D_{2}\right)\{-1\}$. The complex $\overline{\mathcal{F}}\left(D_{1}\right)$ is isomorphic to

$$
0 \longrightarrow \mathcal{F}(D) \stackrel{(\text { id,0) }}{\longrightarrow} \mathcal{F}(D) \oplus \mathcal{F}(D)\{-2\} \longrightarrow 0
$$

so that $\overline{\mathcal{F}}\left(D_{1}\right)$ is isomorphic to the direct sum of a contractible complex and $\mathcal{F}(D)\{-2\}[-1]$.

Since $x\left(D_{1}\right)=x(D)+1=1$ and $y\left(D_{1}\right)=y(D)=0$ for any orientation of $D$, there is an isomorphism $\mathcal{F}\left(D_{1}\right) \cong \mathcal{F}(D)$ in the homotopy category of complexes of bimodules. 


\section{D}

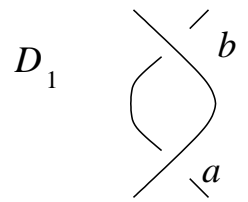

Figure 29: Tangency move
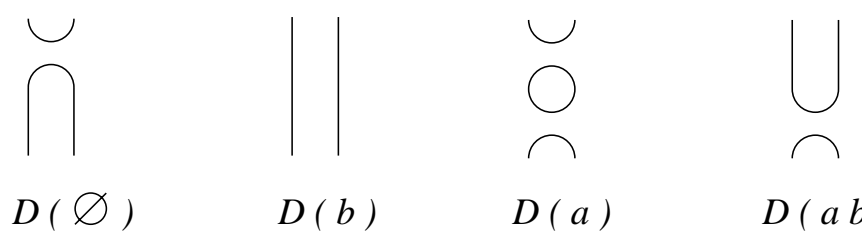

$D(b)$

$D(a)$

$D(a b)$

Figure 30: Four resolutions of $D_{1}$

\subsection{Tangency move}

Let $D$ and $D_{1}$ be two diagrams related by a tangency move (Figure 29). We can assume that $D$ is the diagram Vert ${ }_{2 n}$ of the identity tangle, and $D_{1}=\sigma_{i, 2 n} \sigma_{i, 2 n}^{-1}$. Denote by $a$ and $b$ the crossings of $D$. Notice that $\mathcal{F}(D)$ is isomorphic to $H^{n}$ as an $(n, n)$-bimodule. $D_{1}$ admits four resolutions (Figure 30 ).

$D(\emptyset)$ and $D(a b)$ are isotopic, and $D(a)$ is isotopic to $D(\emptyset)$ with a circle added, so that there are canonical bimodule isomorphisms

$$
\mathcal{F}(D(\emptyset)) \cong \mathcal{F}(D(a b)), \quad \mathcal{F}(D(a)) \cong \mathcal{F}(D(\emptyset)) \otimes \mathcal{A} .
$$

The commutative $\{a, b\}$-cube $V_{D_{1}}$ is actually a commutative square

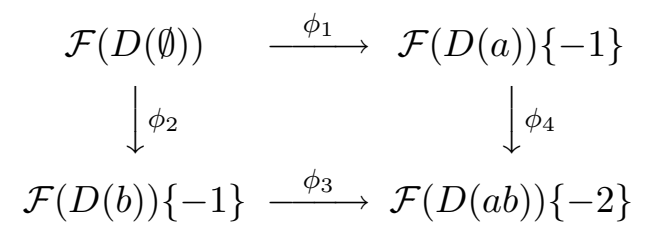

where $\phi_{i}$ are bimodule homomorphisms induced by elementary cobordisms between the four resolutions. The complex $\overline{\mathcal{F}}\left(D_{1}\right)$ is canonically isomorphic to

$$
\begin{aligned}
& \ldots \longrightarrow 0 \longrightarrow \mathcal{F}(D(\emptyset)) \stackrel{\phi_{1}+\phi_{2}}{\longrightarrow} \mathcal{F}(D(a))\{-1\} \oplus \mathcal{F}(D(b))\{-1\} \stackrel{\phi_{4}-\phi_{3}}{\longrightarrow} \\
& \longrightarrow \mathcal{F}(D(a b))\{-2\} \longrightarrow 0 \longrightarrow \ldots
\end{aligned}
$$

Let $\phi: \mathcal{F}(D(b)) \rightarrow \mathcal{F}(D(a))$ be the bimodule homomorphism induced by the Figure 31 cobordism. 


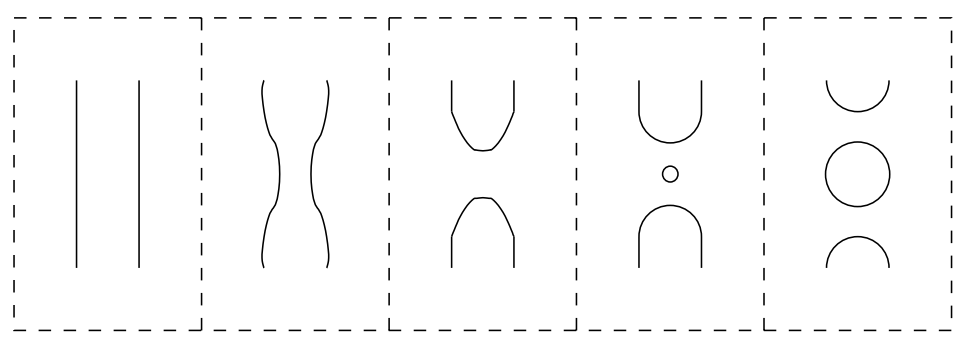

Figure 31: Cobordism for $\phi$

Let $X_{1}$ be the subbimodule of $\mathcal{F}(D(a))\{-1\} \oplus \mathcal{F}(D(b))\{-1\}$ given by $(\phi(u), u)$, for all $u \in \mathcal{F}(D(b))\{-1\}$. This bimodule is isomorphic to $\mathcal{F}(D(b))\{-1\} \cong$ $\mathcal{F}(D)\{-1\}$, and $d X_{1}=0$ since $\phi_{3}=\phi_{4} \phi$ (where $d$ stands for differential in $\left.\overline{\mathcal{F}}\left(D_{1}\right)\right)$. Therefore, $X_{1}$ as a subcomplex of $\mathcal{F}\left(D_{1}\right)$.

Let $X_{2}$ be the subcomplex of $\overline{\mathcal{F}}\left(D_{1}\right)$ generated by $\mathcal{F}(D(\emptyset))$. Since $d$ is injective on $\mathcal{F}(D(\emptyset))$, the complex $X_{2}$ is isomorphic to

$$
0 \longrightarrow \mathcal{F}(D(\emptyset)) \stackrel{\text { id }}{\longrightarrow} \mathcal{F}(D(\emptyset)) \longrightarrow 0,
$$

and, therefore, contractible.

Let $X_{3}$ be the subcomplex of $\overline{\mathcal{F}}\left(D_{1}\right)$ generated by the bimodule

$$
\mathbf{1} \otimes \mathcal{F}(D(\emptyset))\{-1\} \subset \mathcal{A} \otimes \mathcal{F}(D(\emptyset))\{-1\} \cong \mathcal{F}(D(a))\{-1\} .
$$

Since the differential in $\overline{\mathcal{F}}\left(D_{1}\right)$ takes $\mathbf{1} \otimes \mathcal{F}(D(\emptyset))\{-1\}$ bijectively to $\mathcal{F}(D(a b))\{-2\}$, the complex $X_{3}$ is contractible.

Direct sum decomposition $\overline{\mathcal{F}}\left(D_{1}\right)=X_{1} \oplus X_{2} \oplus X_{3}$ implies that complexes $\overline{\mathcal{F}}\left(D_{1}\right)$ and $X_{1}$ are chain homotopic. Therefore, $\overline{\mathcal{F}}\left(D_{1}\right)$ is chain homotopic to $\mathcal{F}(D)[-1]\{-1\}$.

For any orientation, $x\left(D_{1}\right)=1, y\left(D_{1}\right)=1$ and $\mathcal{F}\left(D_{1}\right)=\overline{\mathcal{F}}\left(D_{1}\right)[1]\{1\}$. We obtain a chain homotopy equivalence $\mathcal{F}\left(D_{1}\right) \cong \mathcal{F}(D)$.

\subsection{Triple point move}

Let $D_{1}$ and $D_{2}$ be diagrams $\sigma_{i, 2 n} \sigma_{i+1,2 n} \sigma_{i, 2 n}$ and $\sigma_{i+1,2 n} \sigma_{i, 2 n} \sigma_{i+1,2 n}$, respectively. Denote their double points by $a_{1}, b_{1}, c_{1}, a_{2}, b_{2}, c_{2}$, see Figure 32 . We will construct a chain homotopy equivalence of complexes of bimodules $\mathcal{F}\left(D_{1}\right)$ and $\mathcal{F}\left(D_{2}\right)$. Since $x\left(D_{1}\right)=x\left(D_{2}\right)$ and $y\left(D_{1}\right)=y\left(D_{2}\right)$ it suffices to show that $\overline{\mathcal{F}}\left(D_{1}\right)$ and $\overline{\mathcal{F}}\left(D_{2}\right)$ are homotopy equivalent. 
Note that 1-resolution of the crossing $a_{1}$ of $D_{1}$ is isotopic to 1-resolution of the crossing $a_{2}$ of $D_{2}$, see Figure 33. Consequently, complexes of bimodules associated to these 1-resolutions are isomorphic. These complexes are subcomplexes of $\overline{\mathcal{F}}\left(D_{1}\right)$ and $\overline{\mathcal{F}}\left(D_{2}\right)$, respectively, and will be denoted $Z_{1}$ and $Z_{2}$. As part of this isomorphism there are isomorphisms of bimodules

$$
\begin{aligned}
\mathcal{F}\left(D_{1}\left(a_{1}\right)\right) \cong \mathcal{F}\left(D_{2}\left(a_{2}\right)\right), & & \mathcal{F}\left(D_{1}\left(a_{1} b_{1}\right)\right) \cong \mathcal{F}\left(D_{2}\left(a_{2} b_{2}\right)\right), \\
\mathcal{F}\left(D_{1}\left(a_{1} c_{1}\right)\right) \cong \mathcal{F}\left(D_{2}\left(a_{2} c_{2}\right)\right), & & \mathcal{F}\left(D_{1}\left(a_{1} b_{1} c_{1}\right)\right) \cong \mathcal{F}\left(D_{2}\left(a_{2} b_{2} c_{2}\right)\right) .
\end{aligned}
$$

Resolutions of 0-resolutions of $a_{1}$ and $a_{2}$ are depicted in Figures 34, 35.

Let $\tau_{1}, \tau_{2}$ be bimodule maps associated to Figures 36, 37 cobordisms

$$
\begin{aligned}
& \tau_{1}: \quad \mathcal{F}\left(D_{1}\left(c_{1}\right)\right) \longrightarrow \mathcal{F}\left(D_{1}\left(b_{1}\right)\right), \\
& \tau_{2}: \quad \mathcal{F}\left(D_{2}\left(c_{2}\right)\right) \longrightarrow \mathcal{F}\left(D_{2}\left(b_{2}\right)\right) .
\end{aligned}
$$

Diagrams $D_{1}\left(b_{1}\right)$ and $D_{2}\left(b_{2}\right)$ contain one closed cirle each. Therefore,

$$
\mathcal{F}\left(D_{1}\left(b_{1}\right)\right) \cong \mathcal{A} \otimes \mathcal{F}\left(G_{1}\right), \quad \mathcal{F}\left(D_{2}\left(b_{2}\right)\right) \cong \mathcal{A} \otimes \mathcal{F}\left(G_{2}\right),
$$

where $G_{j}$ is the diagram obtained by removing the circle from $D_{j}\left(b_{j}\right)$. Denote by $M_{j}$ the subbimodule $\mathbf{1} \otimes \mathcal{F}\left(G_{j}\right)$ of $\mathcal{F}\left(D_{j}\left(b_{j}\right)\right)$.

Let $X_{j}^{1}, X_{j}^{2}, X_{j}^{3}$, for $j=1,2$ be the following subcomplexes of $\overline{\mathcal{F}}\left(D_{j}\right)$ :

$$
\begin{aligned}
X_{j}^{1} & =\left\{x+\tau_{j}(x)+y \mid x \in \mathcal{F}\left(D_{j}\left(c_{j}\right)\right)[-1]\{-1\}, y \in Z_{j}\right\}, \\
X_{j}^{2} & =\left\{x+d y \mid x, y \in \mathcal{F}\left(D_{j}(\emptyset)\right)\right\}, \\
X_{j}^{3} & =\left\{x+d y \mid x, y \in M_{j}[-1]\{-1\}\right\},
\end{aligned}
$$

where $d$ denotes the differential in $\overline{\mathcal{F}}\left(D_{j}\right)$.

Proposition 16 (1) $X_{j}^{1}, X_{j}^{2}$, and $X_{j}^{3}$ are indeed subcomplexes of $\overline{\mathcal{F}}\left(D_{j}\right)$.

(2) There is a direct sum decomposition

$$
\overline{\mathcal{F}}\left(D_{j}\right)=X_{j}^{1} \oplus X_{j}^{2} \oplus X_{j}^{3} .
$$

(3) Complexes $X_{j}^{2}$ and $X_{j}^{3}$ are contractible.

(4) Complexes $X_{1}^{1}$ and $X_{2}^{1}$ are isomorphic.

Proof From definition, $X_{j}^{2}, X_{j}^{3}$ are subcomplexes. $X_{j}^{1}$ is a subcomplex since $d\left(x+\tau_{j}(x)\right)$ lies in $Z_{j}$ (we twisted $x$ by $\tau_{j}$ to make it so). Verification of direct sum decompositions is straighforward (or see [38, Section 5.4]). Complexes $X_{j}^{2}$ and $X_{j}^{3}$ are contractible since the differential is injective on $\mathcal{F}\left(D_{j}(\emptyset)\right)$ and $M_{j}[-1]\{-1\}$. The complex $X_{j}^{1}$ is isomorphic to the cone of the map $\mathcal{F}\left(D_{j}\left(c_{j}\right)\right)[-2]\{-1\} \longrightarrow Z_{j}$. Canonical isomorphisms $\mathcal{F}\left(D_{1}\left(c_{1}\right)\right) \cong \mathcal{F}\left(D_{2}\left(c_{2}\right)\right)$ and $Z_{1} \cong Z_{2}$ commute with these maps and give the isomorphism $X_{1}^{1} \cong$ $X_{2}^{1}$. 


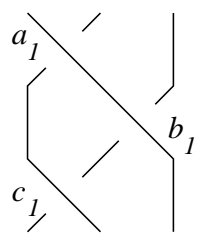

$D_{1}$

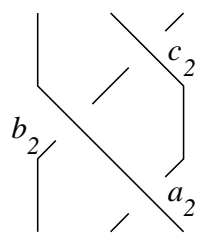

$\mathrm{D}_{2}$

Figure 32: Diagrams $D_{1}$ and $D_{2}$

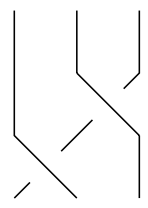

1-resolution of $a_{1}$ 1-resolution of $a_{2}$

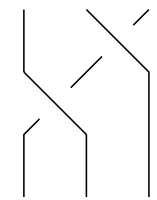

Figure 33: 1-resolutions of $a_{1}$ and $a_{2}$

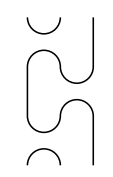

$D_{1}(\varnothing)$

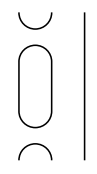

$D_{1}\left(b_{1}\right)$

Figure 34: Resolutions of 0-resolution of $a_{1}$
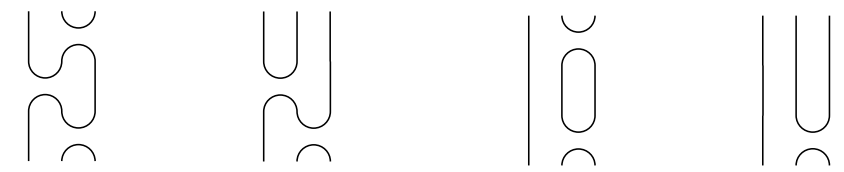

$$
D_{2}(\varnothing)
$$

$$
D_{2}\left(c_{2}\right)
$$$$
D_{2}\left(b_{2}\right)
$$

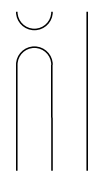

$$
D_{1}\left(b_{1} c_{1}\right)
$$

Figure 35: Resolutions of 0-resolution of $a_{2}$

We obtain a sequence of homotopy equivalences

$$
\overline{\mathcal{F}}\left(D_{1}\right) \cong X_{1}^{1} \cong X_{2}^{1} \cong \overline{\mathcal{F}}\left(D_{2}\right)
$$

Algebraic 83 Geometric Topology, Volume 2 (2002) 


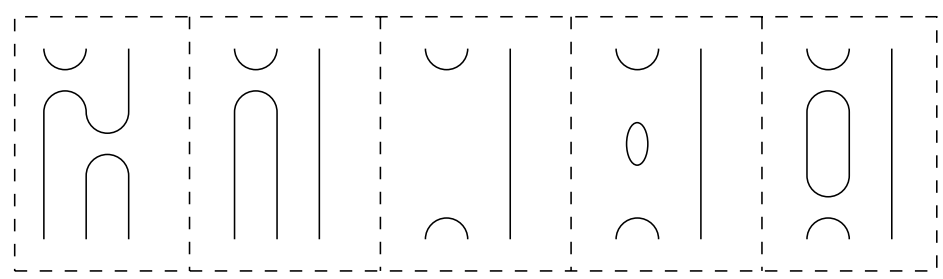

Figure 36: Cobordism for $\tau_{1}$

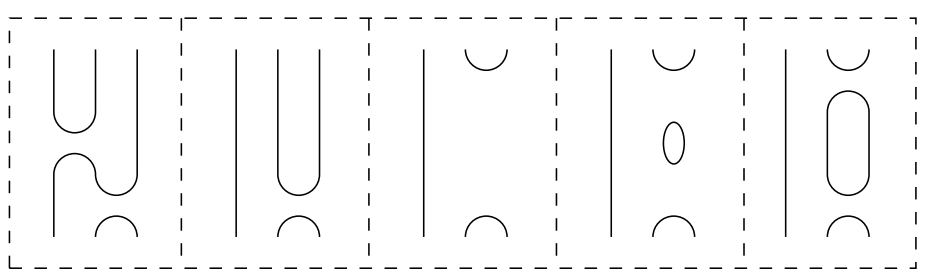

Figure 37: Cobordism for $\tau_{2}$

\section{Interpretations of our invariant}

\subsection{Direct sum decompositions in categories of complexes}

We say that an abelian category $\mathcal{S}$ is Krull-Schmidt if every object is isomorphic to a finite direct sum of indecomposable objects, and this decomposition is unique: for any isomorphism $\oplus_{i \in I} M_{i} \cong \oplus_{j \in J} N_{j}$ between direct sums of indecomposables there is a bijection $f: I \rightarrow J$ such that $M_{i} \cong N_{f(i)}$. The category of finite length modules over a ring is Krull-Schmidt. In particular, the category of finite-dimensional modules over a $k$-algebra $R$ is Krull-Schmidt, where $k$ is a field. Also, the category of finite-dimensional graded modules over a graded $k$-algebra $R$ is Krull-Schmidt.

Let $\operatorname{Kom}(\mathcal{S})$ be the category of bounded complexes of objects of $\mathcal{S}$. It is an abelian category and in the previous sections of this paper we've been working with its quotient category $\mathcal{K}(\mathcal{S})$.

For the rest of this subsection we assume that $\mathcal{S}$ is either the category of finitedimensional modules over a $k$-algebra $R$ or the category of finite-dimensional graded modules over a graded $k$-algebra $R$.

Proposition $17 \operatorname{Kom}(\mathcal{S})$ is Krull-Schmidt.

Proof $\operatorname{Kom}(\mathcal{S})$ is equivalent to the category of finite-dimensional graded (resp. bigraded) modules over the algebra $R \otimes\left(k[\partial] / \partial^{2}=0\right)$. 
Proposition 18 Any object $M$ of $\operatorname{Kom}(\mathcal{S})$ has a direct sum decomposition $M \cong \operatorname{Core}(M) \oplus C t(M)$ where $C t(M)$ is contractible and Core $(M)$ does not contain any contractible direct summands. Core $(M)$ and $C t(M)$ are uniquely (up to an isomorphism) determined by $M$.

We call $\operatorname{Core}(M)$ the core of $M$.

Proposition 19 Complexes $M$ and $N$ in $\operatorname{Kom}(\mathcal{S})$ are chain homotopy equivalent if and only if $\operatorname{Core}(M)$ and $\operatorname{Core}(N)$ are isomorphic.

In other words, two complexes are homotopy equivalent iff they are isomorphic after splitting off their contractible direct summands.

Earlier we proved that the chain homotopy class of $\mathcal{F}(D)$ is an invariant of the tangle $L$. We would like to specialize this invariant to more tangible invariants. One way is to take the cohomology: cohomology groups of $\mathcal{F}(D)$ are graded $(m, n)$-bimodules. The other is to split off contractible summands to get the core of $\mathcal{F}(D)$. Unfortunately, we do not know if $\operatorname{Kom}\left(\mathcal{K}_{n}^{m}\right)$ is a Krull-Schmidt category, i.e. whether it has a unique decomposition property.

Instead, we change from $\mathbb{Z}$ to a field $k$. By tensoring $H^{n}$ and $\mathcal{F}(D)$ with $k$ we get a graded finite-dimensional $k$-algebra, denoted $H_{k}^{n}$, and a complex $\mathcal{F}(D) \otimes k$ of graded $\left(H_{k}^{m}, H_{k}^{n}\right)$-bimodules. Chain homotopy equivalence is preserved by base change, so that the chain homotopy equivalence class of $\mathcal{F}(D) \otimes k$ is an invariant of $L$. In particular, $\operatorname{Core}(\mathcal{F}(D) \otimes k)$ is an invariant of $L$. This invariant is a complex of graded $\left(H_{k}^{m}, H_{k}^{n}\right)$-bimodules, up to an isomorphism.

\subsection{Grothendieck and split Grothendieck groups}

\section{a Grothendieck groups}

The Grothendieck group $G(\mathcal{S})$ of an abelian category $\mathcal{S}$ is an abelian group with generators $[M]$, for all objects $M$ of $\mathcal{S}$, and defining relations $\left[M_{2}\right]=$ $\left[M_{1}\right]+\left[M_{3}\right]$ for all short exact sequences

$$
0 \longrightarrow M_{1} \longrightarrow M_{2} \longrightarrow M_{3} \longrightarrow 0 .
$$

In particular, the Grothendieck group of a Jordan-Gölder category (a category with finite composition series, for instance the category of finite-length modules over a ring), is a free abelian group generated by isomorphism classes of simple objects. 
The Grothendieck group of the category $\mathcal{K}(\mathcal{S})$ of bounded complexes of objects of $\mathcal{S}$ up to chain homotopies is an abelian group with generators $[M]$, for all objects $M$ of $\mathcal{K}(\mathcal{S})$, and defining relations $[M[1]]=-[M]$ (where $M[1]$ is the shift of $M$ one degree to the left, the two different uses of brackets should not lead to confusion), and $\left[M_{2}\right]=\left[M_{1}\right]+\left[M_{3}\right]$ for all short exact sequences of complexes

$$
0 \longrightarrow M_{1} \longrightarrow M_{2} \longrightarrow M_{3} \longrightarrow 0
$$

(that is, $0 \longrightarrow M_{1}^{i} \longrightarrow M_{2}^{i} \longrightarrow M_{3}^{i} \longrightarrow 0$ is exact for all $i$ ).

The inclusion of categories $\mathcal{S} \subset \mathcal{K}(\mathcal{S})$ that to an object $M$ of $\mathcal{S}$ associates the complex

$$
\ldots \longrightarrow 0 \longrightarrow M \longrightarrow 0 \longrightarrow \ldots,
$$

with $M$ in degree 0 , induces an isomorphism between the Grothendieck groups of $\mathcal{S}$ and $\mathcal{K}(\mathcal{S})$.

More generally, the Grothendieck group of a triangulated category $\mathcal{T}$ is an abelian group with generators $[M]$, for all objects $M$ of $\mathcal{T}$, and relations $[M[1]]=-[M]$ and $\left[M_{2}\right]=\left[M_{1}\right]+\left[M_{3}\right]$ for all distinguished triangles

$$
M_{1} \longrightarrow M_{2} \longrightarrow M_{3} \longrightarrow M_{1}[1]
$$

In particular, it is easy to see that the Grothendieck group of the bounded derived category $D^{b}(\mathcal{S})$ is isomorphic to the Grothendieck groups of $\mathcal{K}(\mathcal{S})$ and $\mathcal{S}$.

If $B$ is a graded ring, the Grothendieck group of the category of graded $B$ modules is naturally a $\mathbb{Z}\left[q, q^{-1}\right]$-module, where the multiplication by $q$ corresponds to the grading shift: $[M\{1\}]=q[M]$.

Let $\mathbb{Z}(a)$, for $a \in B^{n}$, be a graded $H^{n}$-module, isomorphic as a graded abelian group to $\mathbb{Z}$, placed in degree 0 , with $1_{a}$ acting as the identity on $\mathbb{Z}(a)$, and $1_{b}$, for $b \neq a$ acting by 0 .

Proposition 20 The Grothendieck group of $H^{n}-\bmod$ is a free $\mathbb{Z}\left[q, q^{-1}\right]$ module generated by $[\mathbb{Z}(a)]$ over all $a \in B^{n}$.

Proof The base change from $\mathbb{Z}$ to $\mathbb{Q}$ is an exact functor from $H^{n}$-mod to the category of graded finite-dimensional $H_{\mathbb{Q}^{n}}^{n}$-modules. By the Jordan-Gölder theorem the Grothendieck group of the latter is a free $\mathbb{Z}\left[q, q^{-1}\right]$-module spanned by isomorphism classes of simple $H_{\mathbb{Q}}^{n}$-modules. The base change defines a bijection between modules $\mathbb{Z}(a)$ and simple $H_{\mathbb{Q}}^{n}$-modules. Therefore, images $[\mathbb{Z}(a)]$ of 
modules $\mathbb{Z}(a)$ in the Grothendieck group of $H^{n}$-mod are linearly independent over $\mathbb{Z}\left[q, q^{-1}\right]$.

Any module in $H^{n}$-mod has a finite-length composition series with subsequent quotients isomorphic to $\mathbb{Z}(a)\{i\}$ and $\mathbb{Z}(a) / p \mathbb{Z}(a)\{i\}$, for various $a \in B^{n}$, primes $p$, and integers $i$. The images of modules $\mathbb{Z}(a) / p \mathbb{Z}(a)$ are zero in the Grothendieck group. Therefore, the Grothendieck group of $H^{n}$-mod is generated, as a $\mathbb{Z}\left[q, q^{-1}\right]$-module, by $[\mathbb{Z}(a)]$, over all $a \in B^{n}$.

Given a subcategory $\mathcal{C}$ of $\mathcal{S}$, or $\mathcal{K}(\mathcal{S})$, or $D^{b}(\mathcal{S})$, define the Grothendieck group $G(\mathcal{C})$ of $\mathcal{C}$ as the subgroup of the Grothendieck group of the larger category generated by $[M]$ over all objects $M$ of $\mathcal{C}$.

To summarize, we have:

Proposition 21 (1) The Grothendieck groups of $H^{n}-\bmod , D^{b}\left(H^{n}-\bmod \right)$, and $\mathcal{K}^{n}$ are naturally isomorphic. They are free $\mathbb{Z}\left[q, q^{-1}\right]$-modules generated by $[\mathbb{Z}(a)]$, for $a \in B^{n}$.

(2) The Grothendieck groups of $H_{P}^{n}$-mod and $\mathcal{K}_{P}^{n}$ are naturally isomorphic. They are free $\mathbb{Z}\left[q, q^{-1}\right]$-modules generated by $\left[P_{a}\right]$, for $a \in B^{n}$.

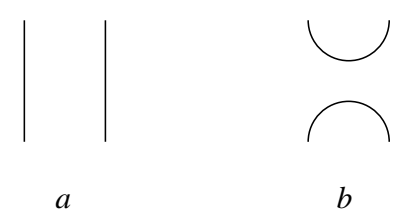

Figure 38: Flat tangles $a$ and $b$.

Let $a$ and $b$ be flat $(1,1)$-tangles depicted in Figure 38. Bimodules $\mathcal{F}(a)$ and $\mathcal{F}(b)$ are isomorphic to $H^{1}$ and $H^{1} \otimes H^{1}\{-1\}$, respectively (note that $\left.H^{1} \cong \mathcal{A}\{1\}\right)$. There is a short exact sequence of bimodules

$$
0 \longrightarrow \mathcal{F}(a)\{2\} \longrightarrow \mathcal{F}(b)\{1\} \longrightarrow \mathcal{F}(a) \longrightarrow 0
$$

isomorphic to the exact sequence

$$
0 \longrightarrow H^{1}\{2\} \stackrel{\gamma}{\longrightarrow} H^{1} \otimes H^{1} \stackrel{m}{\longrightarrow} H^{1} \longrightarrow 0
$$

where $\gamma(\mathbf{1})=\mathbf{1} \otimes X-X \otimes \mathbf{1}$. Therefore, in the Grothendieck group of graded $\left(H^{1}, H^{1}\right)$-bimodules we have

$$
[\mathcal{F}(b)]=\left(q+q^{-1}\right)[\mathcal{F}(a)] .
$$




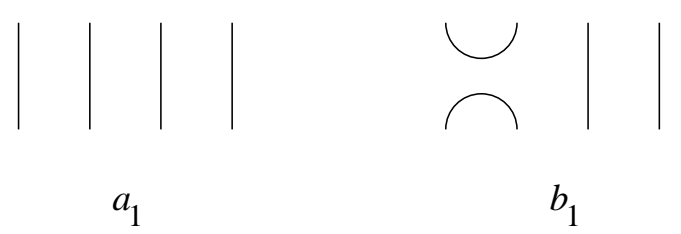

Figure 39: Stabilization of $a$ and $b$

On the other hand, we would like bimodules $\mathcal{F}(a)$ and $\mathcal{F}(b)$ to be independent in the Grothendieck group, by analogy with the linear Temperley-Lieb category $\mathcal{L} \mathcal{T} \mathcal{L}$, where $a$ and $b$ are linearly independent over $\mathbb{Z}\left[q, q^{-1}\right]$ as morphisms from 1 to 1 . For this purpose we use split Grothendieck groups, described below.

Remark Stabilizing $a$ and $b$ also makes them independent. Let $a_{1}$ and $b_{1}$ be flat tangles obtained from $a$ and $b$ by adding $2 l$ vertical lines, as in Figure 39 . Bimodules $\mathcal{F}\left(a_{1}\right)$ and $\mathcal{F}\left(b_{1}\right)$ are independent in the Grothendieck groups of finitely-generated $\left(H^{l+1}, H^{l+1}\right)$-bimodules, for $l>0$. This dependence property is a fancier version of the fact that the linear map

$$
\operatorname{Hom}_{\mathfrak{s l}(2)}\left(V^{\otimes 2 n}, V^{\otimes 2 m}\right) \longrightarrow \operatorname{Hom}_{\mathbb{C}}\left(\operatorname{Inv}\left(V^{\otimes 2 n}\right), \operatorname{Inv}\left(V^{\otimes 2 m}\right)\right)
$$

which restricts an $\mathfrak{s l}(2)$-intertwiner between tensor powers of the fundamental representation to the map between the spaces of $\mathfrak{s l}(2)$-invariants is not injective, in general, but becomes injective after a stabilization with the identity map of $V^{\otimes 2 l}$, for $l>n, m$.

\section{b Split Grothendieck groups and the Kauffman bracket}

The split Grothendieck group $G^{\mathrm{spl}}(\mathcal{S})$ of an additive category $\mathcal{S}$ is the abelian group with generators $[M]$ for all objects $M$ of $\mathcal{S}$ and relations $\left[M_{1}\right]=\left[M_{2}\right]+$ $\left[M_{3}\right]$ whenever $\left[M_{1}\right]$ is isomorphic to the direct sum of $M_{2}$ and $M_{3}$.

The split Grothendieck group tends to be much larger than the Grothendieck group. For instance, if the category $\mathcal{S}$ is Krull-Schmidt the split Grothendieck group of $\mathcal{S}$ is an abelian group freely generated by isomorphism classes of indecomposable objects of $\mathcal{S}$.

Proposition $22[\mathcal{F}(a)]$, over all $a \in B_{n}^{m}$, are independent over $\mathbb{Z}\left[q, q^{-1}\right]$, when treated as elements of the split Grothendieck group of the category of finitely-generated graded $\left(H^{m}, H^{n}\right)$-bimodules.

Proof Tensor everything with a field $k$. The category of finitely-generated graded $\left(H_{k}^{m}, H_{k}^{n}\right)$-bimodules is Krull-Schmidt. According to Proposition 8, modules $\mathcal{F}(a) \otimes k$ are indecomposable and pairwise non-isomorphic. 
Therefore, the split Grothendieck group $G^{\mathrm{spl}}\left(\mathcal{S}_{n}^{m}\right)$ of the category $\mathcal{S}_{n}^{m}$ of geometric $(m, n)$-bimodules is a free $\mathbb{Z}\left[q, q^{-1}\right]$-module with a basis $\{[\mathcal{F}(a)], a \in$ $\left.B_{n}^{m}\right\}$. Thus, the split Grothendieck group is canonically isomorphic to the $\mathbb{Z}\left[q, q^{-1}\right]$-module of morphisms from $n$ to $m$ in the linear Temperley-Lieb category. The isomorphism takes $[\mathcal{F}(a)]$ to $a$. Denote this isomorphism by iso:

$$
\text { iso : } G^{\mathrm{spl}}\left(\mathcal{S}_{n}^{m}\right) \stackrel{\cong}{\longrightarrow} \operatorname{Mor}_{\mathcal{L} \mathcal{L} \mathcal{L}}(n, m) \text {. }
$$

Note that iso takes the tensor product of bimodules to the composition of morphisms. We can restate this observation in the language of 2-categories.

Proposition 23 iso is an equivalence between the split Grothendieck category of $\mathbb{G} \mathbb{B}$, the 2-category of geometric $H$-bimodules, and the linear Temperley-Lieb category $\mathcal{L} \mathcal{T} \mathcal{L}$.

To a complex $M \in \mathrm{Ob}\left(\mathcal{K}_{n}^{m}\right)$ of geometric $(m, n)$-bimodules assign $[M]=$ $\sum_{i}(-1)^{i}\left[M^{i}\right]$, an element of the split Grothendieck group of $\mathcal{S}_{n}^{m}$.

Proposition 24 Let $L$ be an oriented $(m, n)$-tangle. Isomorphism iso takes $[\mathcal{F}(L)]$ to the Kauffman bracket $K(L)$.

Proof Immediate from our definition of $\mathcal{F}(L)$.

\subsection{Functor interpretations of the invariant}

Our invariant of an $(m, n)$-tangle $L$ is a chain isomorphism class of the complex $\mathcal{F}(L)$ of geometric $(m, n)$-bimodules, equivalently, an isomorphism class of the object $\mathcal{F}(L)$ of $\mathcal{K}_{n}^{m}$.

There are at least 4 ways to turn this complex into a functor. Namely, tensoring with $L$ is a functor

- between categories $\mathcal{K}^{n}$ and $\mathcal{K}^{m}$,

- between categories $\mathcal{K}_{P}^{n}$ and $\mathcal{K}_{P}^{m}$,

- between derived categories $\mathcal{D}^{n}$ and $\mathcal{D}^{m}$ (where we denoted by $\mathcal{D}^{n}$ the bounded derived category $D^{b}\left(H^{n}\right.$-mod) $)$,

- Between stable categories $H_{k}^{n}-\underline{\bmod }$ and $H_{k}^{n}-\underline{\bmod }$ (see the end of Section 6.7). 
There are obvious inclusion and localization functors

$$
\mathcal{K}_{P}^{n} \stackrel{\psi_{1}}{\longrightarrow} \mathcal{K}^{n} \stackrel{\psi_{2}}{\longrightarrow} D^{n} \text {. }
$$

The inclusion functor $\psi_{1}$ is fully faithful, but the localization functor $\psi_{2}$ is neither full (surjective on morphisms) nor faithful (injective on morphisms). The composition $\psi_{2} \psi_{1}$ is fully faithful and makes $\mathcal{K}_{P}^{n}$ a full subcategory of $D^{n}$. Functors $\mathcal{F}(L)$, for $(m, n)$-tangles $L$, commute with functors $\psi_{1}$ and $\psi_{2}$ :

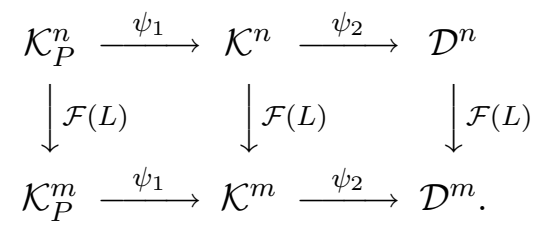

The functor $\psi_{1}$ induces an inclusion of Grothendieck groups, which is proper for $n>0$ (see Section 6.7), while $\psi_{2}$ induces an isomorphism of Grothendieck groups.

Remark There is a natural way to identify the Grothendieck group $G\left(\mathcal{K}^{n}\right)$ with a $\mathbb{Z}\left[q, q^{-1}\right]$-submodule of $\operatorname{Inv}(n)$. Here $\operatorname{Inv}(n)$ is the space of $U_{q}\left(\mathfrak{s l}_{2}\right)$ invariants in $V^{\otimes 2 n}$, the $2 n$-th tensor power of the fundamental representation. Identify generators $[\mathbb{Z}(a)]$ of $G\left(\mathcal{K}^{n}\right)$ with canonical basis vectors $\operatorname{in} \operatorname{Inv}(n)$ (see [23], [37] for a study of Lusztig canonical and dual canonical bases in this space). Then images $\left[P_{a}\right]$ of indecomposable projective $H^{n}$-modules go to dual canonical basis vectors in $\operatorname{Inv}(n)$. This correspondence intertwines actions of the category of tangles on $G\left(\mathcal{K}^{n}\right)$ via $[\mathcal{F}(L)]$ and on $\operatorname{Inv}(n)$ via $J^{\prime}(L)$ (see the introduction for the latter notation).

\subsection{Categories and 2-categories}

Let $\mathbb{K}$ be the 2-category with nonnegative integers as objects and $\mathcal{K}_{n}^{m}$ as the category of 1-morphisms between $n$ and $m$. Thus, 1-morphisms of $\mathbb{K}$ from $n$ to $m$ are defined as objects of $\mathcal{K}_{n}^{m}$ and 2-morphisms of $\mathbb{K}$ are morphisms of $\mathcal{K}_{n}^{m}$. One can think of $\mathbb{K}$ as the chain homotopy 2-category of the 2-category $\mathbb{G B}$ of geometric $H$-bimodules.

Our main categories and 2-categories can be collected into a commutative diagram:

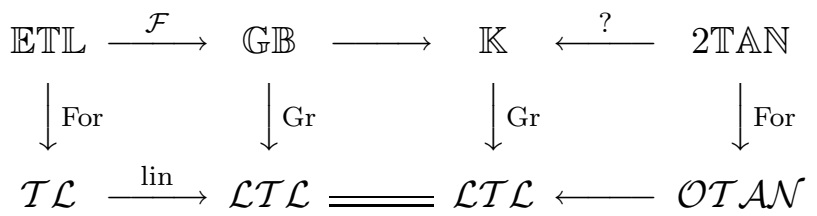


$\mathbb{E T L}$ is the Euler-Temperley-Lieb 2-category, defined in Section 2.3. $2 \mathbb{T} \mathbb{A N}$ is the 2-category of oriented and suitably decorated tangle cobordisms (2-tangles). The categories $\mathcal{T} \mathcal{L}, \mathcal{L} \mathcal{T}$, and $\mathcal{O} \mathcal{T} \mathcal{A N}$ are defined in Sections 2.2, 2.2, and 3.1, respectively.

Vertical arrows labelled Gr denote the passage to the (split) Grothendieck category of a 2-category. Vertical arrows labelled For forget 2-morphisms and identify isomorphic 1-morphisms of a 2-category . The result is a category.

The 2-functor $\mathcal{F}$ was discussed in Section 2.9, The 2-functor $\mathbb{G} \mathbb{B} \rightarrow \mathbb{K}$ is the inclusion of 2-categories which comes from embeddings of categories $\mathcal{S}_{n}^{m} \subset \mathcal{K}_{n}^{m}$.

What we really are after is the 2-functor from $2 \mathbb{T} \mathbb{A}$ to $\mathbb{K}$ denoted by the question mark. The construction of this functor will be the subject of a followup paper.

\section{Biadjoint functors, Frobenius algebras, and ex- tended topological quantum field theories}

\subsection{Topological quantum field theories}

An $n$-dimensional topological quantum field theory (TQFT, for short) is a tensor functor from the category of $n$-dimensional oriented cobordisms to an additive tensor category ${ }^{1} T$. A TQFT associates an object $F(M)$ of the category $T$ to a closed oriented $(n-1)$-manifold $M$ and a map

$$
F(N): F(M) \rightarrow F\left(M^{\prime}\right)
$$

\footnotetext{
${ }^{1}$ In all known examples of TQFTs only the following additive tensor categories appear:

(1) The category of finite dimensional vector spaces over a field.

(2) The category of bounded complexes of free abelian groups of finite rank (or of finite-dimensional vector spaces) up to chain homotopy.

(3) Mild variations of 1 and 2 .

In the original Atiyah's definition [4] the target category for a TQFT is the category of modules over a (commutative) ring $\Lambda$. This works well for 3 -dimensional TQFTs, but not for the 4-dimensional ones. In the Floer-Donaldson 4-dimensional TQFT the target category is the category of $\mathbb{Z}_{8}$-periodic complexes up to chain homotopies of free abelian groups of finite rank. To keep dimensions 3 and 4 under the same roof we weaken Atiyah's definition and only request that the target category is an additive tensor category.
} 
to an oriented $n$-cobordism $N$ with the boundary $M \sqcup\left(-M^{\prime}\right)$. The condition that $F$ is a tensor functor means, among other things, that

(1) $\quad F\left(M \sqcup M^{\prime}\right) \cong F(M) \otimes F\left(M^{\prime}\right)$ and $F\left(N \sqcup N^{\prime}\right)=F(N) \otimes F\left(N^{\prime}\right)$ for closed oriented $(n-1)$-manifolds $M, M^{\prime}$ and oriented $n$-cobordisms $N, N^{\prime}$.

(2) Reversal of the orientation matches the duality in the category $T$ :

$$
F(-M) \cong F(M)^{*} .
$$

(3) $F(\emptyset)=\underline{1}$. To the empty $(n-1)$-manifold we associate the unit object $\underline{1}$ of $T$.

(4) If $N$ is a closed $n$-manifold, $F(N)$ is a morphism $\underline{1} \rightarrow \underline{1}$. In typical examples, $\operatorname{Hom}_{T}(\underline{1}, \underline{1})$ is the base field or $\mathbb{Z}$, so that $F(N)$ is a field-valued or an integer-valued invariant. To the empty $n$-manifold we associate the identity map of $\underline{1}$.

(5) $F\left(N \circ N^{\prime}\right)=F(N) \circ F\left(N^{\prime}\right)$ where $\circ$ on the LHS is the composition of cobordisms and on the RHS of morphisms.

This definition of a TQFT is unnecessarily restrictive. In practice, we allow more flexibility by enriching the category of oriented cobordisms with extra algebraic data. For instance, in the Witten-Reshetikhin-Turaev theory (see $[57],[48])$ an object is a closed oriented surface together with a fractional framing of the stabilized tangent bundle.

Quite often the situation is even more complicated. The category of cobordisms is enhanced and then certain objects and/or cobordisms are excluded from the category. Thus, in the Donaldson-Floer theory an object is an admissible $S O(3)$-bundle $Q$ over a 3-manifold $M$, (admissible $=M$ is a homology sphere or $Q$ has no reducible flat connections [11]). The admissibility condition sharply limits pairs $(Q, M)$ allowed as objects.

We refer to generalizations of the first kind as decorated TQFTs, of the second kind as restricted (and decorated) TQFTs.

An $n$-dimensional genus 0 TQFT is a tensor functor from the category of $(n-2)$-dimensional oriented cobordisms in $\mathbb{R}^{n}$ to an additive tensor category. A plentitude of interesting examples exists in dimension 3, in which case the category of cobordisms is usually called the category of tangles. A genus 0 three-dimensional TQFT can be assigned to each finite-dimensional complex simple Lie algebra $\mathfrak{g}$ and an irreducible representation $V$ of $\mathfrak{g}$ [55]. In this paper and its predecessor [38] we work towards constructing a 4-dimensional genus 0 TQFT. 


\subsection{TQFT with corners}

An $n$-dimensional TQFT with corners associates an additive category $F(K)$ to a closed oriented $(n-2)$-manifold $K$, a functor $F(M): F\left(\partial_{0} M\right) \rightarrow F\left(\partial_{1} M\right)$ to an oriented $(n-1)$-cobordism $M$, and a natural transformation $F(N)$ : $F\left(\partial_{0} N\right) \longrightarrow F\left(\partial_{1} N\right)$ of functors to an oriented $n$-cobordism $N$ with corners. This assignment is subject to a wealth of conditions, two of which are

- $F$ is a 2-functor from the 2-category $\mathbb{M C}_{n}$ of oriented $n$-cobordisms with corners to the 2-category $\mathbb{A C}$ of additive categories.

- $F$ restricts to an $n$-dimensional TQFT. Namely, the category $F(\emptyset)$ assigned to the empty $(n-2)$-manifold is an additive tensor category. A closed oriented $(n-1)$-manifold $M$ is a cobordism between the empty manifolds, so that $F(M)$ is a functor in the category $F(\emptyset)$. Applied to the unit object of $F(\emptyset)$ this functor produces an object of $F(\emptyset)$ (call this object $\widetilde{F}(M))$. For an $n$-cobordism $N$ between closed $(n-1)$-manifolds, $F(N)$ is a natural transformation between functors $F\left(\partial_{0} N\right)$ and $F\left(\partial_{1} N\right)$. Evaluated at the unit object of $F(\emptyset)$ this natural transformation is a morphism

$$
\widetilde{F}(N): \widetilde{F}\left(\partial_{0} N\right) \rightarrow \widetilde{F}\left(\partial_{1} N\right) .
$$

Varying $M$ and $N$ we obtain an $n$-dimensional TQFT.

Some other conditions, often taken for granted in the literature, such as

- $F$ is tensor on objects: for $(n-2)$-manifolds $K_{1}, K_{2}$ the category $F\left(K_{1} \sqcup\right.$ $\left.K_{2}\right)$ is isomorphic to the tensor product of categories $F\left(K_{1}\right)$ and $F\left(K_{2}\right)$,

- $F(K)$ are semisimple categories, for all $(n-2)$-manifolds $K$,

seem to us too ambiguous or restrictive. Sophisticated examples of combinatorially defined TQFTs (with or without corners) have been found in dimension 3 only, including the Witten-Reshetikhin-Turaev 3D TQFT with corners and its generalizations from $\mathfrak{s l}_{2}$ to other simple Lie algebras. In the WittenReshetikhin-Turaev TQFT the categories associated to closed $(n-2)$-manifolds (i.e. 1-manifolds) are semisimple, but they aren't in the 2D TQFT with corners associated to Frobenius algebras (Section 6.4 treats this toy yet illuminating example), and they should not be semisimple in the yet-to-be-found $4 \mathrm{D}$ TQFT with corners (Section 6.5). Likewise, once the semisimplicity condition is waived, defining the tensor product of additive categories in an abstract way becomes rather hard, and we feel that the condition that $F$ is tensor on objects is best to be left out for now. 


\subsection{Biadjoint functors and TQFT with corners}

To an $(n-1)$-cobordism $M$ between closed $(n-2)$-manifolds $K_{1}$ and $K_{2}$ a TQFT with corners assigns a functor $F(M)$ between categories $F\left(K_{1}\right)$ and $F\left(K_{2}\right)$ (for simplicity, we will ignore orientations in our discussion). Denote by $W$ the cobordism $M$ considered as a cobordism from $K_{2}$ to $K_{1}$. There is a canonical "contraction" $n$-cobordism between $M W$ and the $(n-1)$-cobordism $K_{2} \times[0,1]$, and another canonical "contraction" $n$-cobordism between $W M$ and $K_{1} \times[0,1]$. Figures 40-42 show how to construct these cobordisms. $M$ is depicted by an interval, thickened in one place to emphasize nontrivial topology of $M$. Multiply $M$ by $[0,1]$ and then contract $K_{2} \times[0,1]$ to $K_{2}$. After that fan out, turning $K_{1} \times[0,1]$ into $K_{1} \times$ semicircle, and add corners. Dashed lines show copies of $M$ inside the fan. The result is an $n$-cobordism between $W M$ and $K_{1} \times[0,1]$.

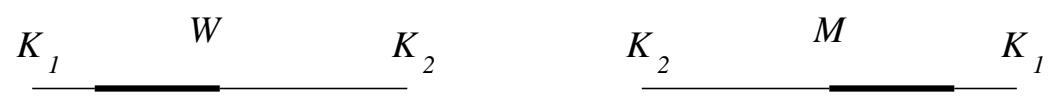

Figure 40: Cobordisms $W$ and $M$
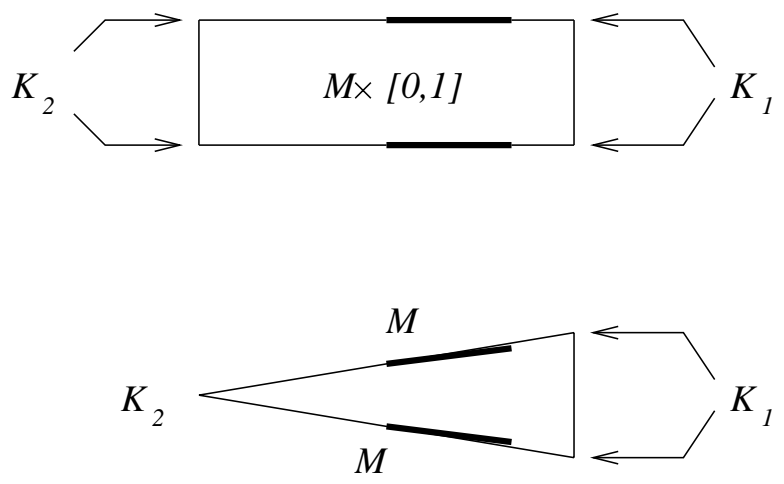

Figure 41: Multiply $M$ by $[0,1]$ and contract $K_{2} \times[0,1]$ to $K_{2}$

TQFT with corners assigns natural transformations

$$
\begin{array}{ll}
\operatorname{Id}_{F\left(K_{1}\right)} \longrightarrow F(W) F(M), & F(W) F(M) \longrightarrow \operatorname{Id}_{F\left(K_{1}\right)}, \\
\operatorname{Id}_{F\left(K_{2}\right)} \longrightarrow F(M) F(W), & F(M) F(W) \longrightarrow \operatorname{Id}_{F\left(K_{2}\right)}
\end{array}
$$

to these $n$-cobordisms. Relations between these natural transformations say that the functor $F(W)$ is left and right adjoint to $F(M)$. We will say that $F(W)$ is a two-sided adjoint or a biadjoint functor of $F(M)$. A functor which has a biadjoint is often called a Frobenius functor. 

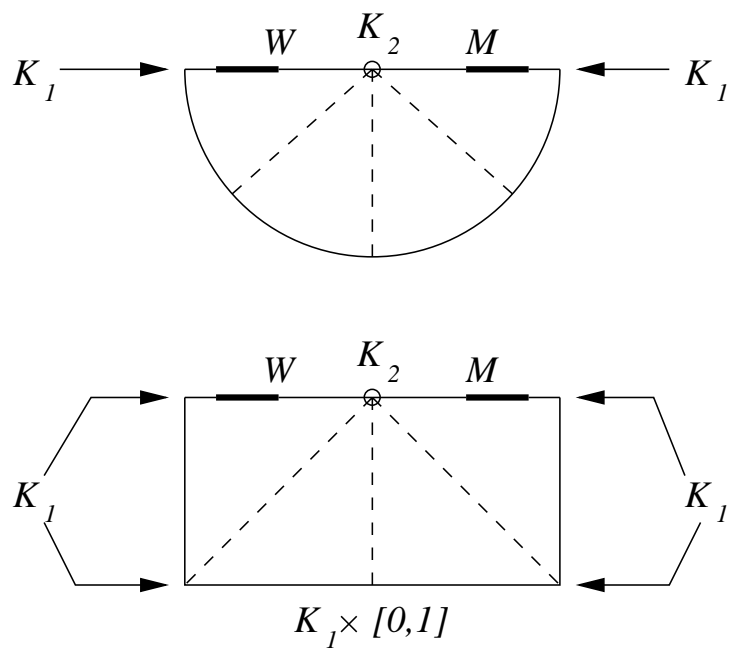

Figure 42: Fan out and add corners

Proposition 25 For any $(n-1)$-cobordism $M$ and any $n$-dimensional TQFT with corners $F$ the functor $F(M)$ has a biadjoint.

This rather tautological observation is a powerful hint where to search for TQFTs with corners (of course, we are primarily interested in the four-dimensional ones):

Find categories with many Frobenius functors.

Our favorite examples are:

(1) Categories of modules over symmetric and Frobenius algebras and their derived categories.

(2) Categories of highest weight modules over simple Lie algebras and their derived categories.

(3) Derived categories of coherent sheaves on Calabi-Yau manifolds.

(4) Fukaya-Floer categories of lagrangians in a symplectic manifold.

We discuss these examples at length below. As a warm-up, notice that the composition of two Frobenius functors is a Frobenius functor, and that invertible functors are Frobenius.

1a Symmetric algebras Let $R$ be a commutative ring and $A$ an $R$-algebra. $A$ is called a symmetric $R$-algebra if 
- $A$ is a finitely-generated projective $R$-module,

- $A$ and $A^{*} \stackrel{\text { def }}{=} \operatorname{Hom}_{R}(A, R)$ are isomorphic as $A$-bimodules.

If $R$ is a field, an $R$-algebra $A$ is symmetric if and only if it is finite-dimensional over $R$ and there is an $R$-linear functional $\operatorname{Tr}: A \rightarrow R$ which is nondegenerate $(\operatorname{Tr}(x A)=0 \Rightarrow x=0)$ and symmetric $(\operatorname{Tr}(x y)=\operatorname{Tr}(y x)$ for all $x, y \in A)$.

Examples of symmetric algebras include

- matrix algebras,

- group algebras of finite groups,

- Hecke algebras of finite root systems,

- cyclotomic Hecke algebras [45],

- finite-dimensional quantum groups $U_{q}(\mathfrak{g}), q$ a root of unity,

- the Drinfeld double of a finite-dimensional Hopf algebra [31, Theorem 6.10],

- rings $H^{n}$ and algebras $A^{n}$ (see Sections 2.4, 6.4 and Propositions 32,30),

- trivial extension algebras [43, Proposition 16.60],

- commutative Frobenius algebras (see Section 6.4).

For reasons explained in Section 6.5 we will disregard semisimple symmetric algebras in favour of the nonsemisimple ones. The matrix algebras over a field are semisimple. The group algebra $k[G]$ of a finite group $G$ is nonsemisimple when the characteristic of the field $k$ divides the order of $G$. The Hecke algebra $H_{n, q}$ of the root system $A_{n-1}$ is nonsemisimple if $q \neq 1$ is a root of unity of order at most $n$, interesting examples of cyclotomic Hecke algebras are nonsemisimple [2], commutative Frobenius algebras over $\mathbb{C}$ are nonsemisimple except when isomorphic to $\mathbb{C}^{\oplus n}$. Other algebras in the above list are nonsemisimple except for several obvious cases. For many examples of semisimple symmetric algebras, not covered in the list above, we refer the reader to [31].

Warning Algebras of polynomials are not symmetric according to our definition. However, they are sometimes called symmetric since, as vector spaces, they are isomorphic to the direct sum of all symmetric powers of a vector space. We will avoid this usage to escape possible confusion.

Our interest in symmetric algebras is motivated, in particular, by the following:

Proposition 26 If $A_{1}$ and $A_{2}$ are symmetric algebras, the functor of tensoring with a sweet $\left(A_{2}, A_{1}\right)$-bimodule $N$ admits a biadjoint functor (tensoring with $N^{*}$ ). 
See [49] for a proof.

Examples of such functors are

- Induction and restriction functors in finite Hecke algebras, cyclotomic Hecke algebras [2], group algebras of finite groups, finite-dimensional quantum groups $U_{q}(\mathfrak{g})$, and direct summands of these functors.

- The functor of tensoring with a finite-dimensional representation of a group algebra, or a finite quantum group, and direct summands of these functors.

- Tensoring with $(m, n)$-bimodules $\mathcal{F}(b)$, for diagrams $b \in \widehat{B}_{n}^{m}$ (see Section 2.6). These bimodules are left $H^{m}$ - and right $H^{n}$-projective. The tensor product with $\mathcal{F}(b)$ functor, treated as a functor between categories of $H^{m}$ - and $H^{n}$-modules, rather then as a functor between categories of graded modules, has a biadjoint functor - tensoring with $\mathcal{F}(W(b))$.

- Tensoring with $\left(A^{m}, A^{n}\right)$-bimodules $F_{A}(b)$, for $b \in \widehat{C}_{n}^{m}$ (see Section 6.4).

More generally, if $(N, d)$ is a bounded complex of sweet $\left(A_{2}, A_{1}\right)$-bimodules, the functor of tensoring with $(N, d)$, considered as a functor between derived categories, or as a functor between categories of complexes up to chain homotopies, admits a biadjoint [49]. If $D$ is a diagram of an $(m, n)$-tangle, tensoring with the complex $\mathcal{F}(D)$ is a functor betweeen derived categories or chain homotopy categories of $H^{n}, H^{m}$-modules. It has a biadjoint functor-tensoring with $\mathcal{F}\left(D^{!}\right)$, where $D^{!}$is the mirror image of $D$ (when we work with modules which are not graded).

1b Frobenius algebras These are close relatives of symmetric algebras. An $R$-algebra $A$ is called Frobenius over $R$ if the restriction functor $A$-mod $\longrightarrow$ $R$-mod has a 2 -sided adjoint functor. This amounts to the condition that induction and coinduction functors Ind, Coind $: R$-mod $\longrightarrow A$-mod given by

$$
\operatorname{Ind}(M)=A \otimes_{R} M, \quad \operatorname{Coind}(M)=\operatorname{Hom}_{R}(A, M)
$$

are isomorphic. We refer the reader to [31, Section 1.3] for a detailed discussion. If $R$ is a field, $A$ is Frobenius iff there is a nondegenerate functional $\operatorname{Tr}: A \longrightarrow$ $R$, i.e. $\operatorname{Tr}$ is $R$-linear and $\operatorname{Tr}(x A)=0$ implies $x=0$ for $x \in A$.

Notice the difference between symmetric and Frobenius algebras over a field: a symmetric algebra admits a symmetric nongenerate functional, $\operatorname{Tr}(x y)=$ $\operatorname{Tr}(y x)$. In particular, any symmetric algebra is Frobenius. Examples of Frobenius, but not, in general, symmetric, algebras are

- universal enveloping algebras of restricted Lie algebras [7], 
- finite-dimensional Hopf algebras,

- NilCoxeter algebras [36],

- 0-Hecke algebras [17],

- cohomology algebra $H^{*}(M, R)$ of a closed oriented manifold $M$, where $R$ is a field. $H^{*}(M, R)$, however, is a symmetric superalgebra,

- algebras $\operatorname{Ext}^{*}(\mathcal{G}, \mathcal{G})$ where $\mathcal{G}$ is a coherent sheaf on a Calabi-Yau variety (a smooth projective algebraic variety with the trivial canonical class).

If $A_{1}$ and $A_{2}$ are Frobenius algebras and $N$ a sweet $\left(A_{1}, A_{2}\right)$-bimodule then $N$ is quite often Frobenius even when $A_{1}$ and $A_{2}$ are not symmetric.

For instance, let $A$ be a Hopf algebra and $V$ a finite-dimensional representation of $A$. The representation $V$ have left and right dual representations $V^{*}$ and $V^{\circ}$, both isomorphic as vector spaces to $\operatorname{Hom}_{\mathbb{C}}(V, \mathbb{C})$, but with different left $A$-module structures:

$$
\begin{array}{rr}
a f(x)=f(S(a) x), & a \in A, f \in V^{*}, x \in V \\
a f(x)=f\left(S^{-1}(a) x\right), & a \in A, f \in V^{\circ}, x \in V,
\end{array}
$$

where $S$ is the antipode of $A$.

The functor $T_{V}(M)=V \otimes M$ of tensoring (over the ground field) an $A$-module on the left with $V$ has a left adjoint functor $T_{V^{*}}$ and a right adjoint functor $T_{V^{\circ}}$. If $S^{2}$ is an inner automorphism of $H$ then $V^{*}$ and $V^{\circ}$ are isomorphic as $H$-modules, and the functor $T_{V}$ is Frobenius. Examples are:

- $S^{2}=$ Id in any commutative or cocommutative Hopf algebra [54, Proposition 4.0.1]. Functors $T_{V}$ and their direct summands (in particular, translation functors) are used extensively to study representations of these Hopf algebras [29].

- $S^{2}$ is an inner automorphism in the quantum group $U_{q}(\mathfrak{g})$.

Any finite-dimensional Hopf algebra $A$ is Frobenius, but not necessarily symmetric. Even if $S^{2}$ is inner, $A$ might not be symmetric. For instance, the universal enveloping algebra of a restricted Lie algebra $\mathfrak{g}$ is symmetric if and only if $\operatorname{tr}(\operatorname{ad}(x))=0$ for any $x \in \mathfrak{g}[20]$.

The functor $T_{V}$ is equivalent to the functor of tensoring (over $A$ ) with a sweet $A$-bimodule $V \otimes A$. Often $T_{V}$ decomposes into direct sum of many functors, each of them Frobenius. Thus, for $A$ a universal enveloping algebra of a restricted Lie algebra there are quite a few Frobenius functors in the category of $A$-modules. 
Remark If $A_{1}, A_{2}$ are arbitrary rings and $N$ an $\left(A_{1}, A_{2}\right)$-bimodule, $N$ is called a Frobenius bimodule if the tensor product functor $N \otimes$ ? : $A_{2}$-mod $\longrightarrow$ $A_{1}$-mod admits a biadjoint, see [31, Chapter 2] for more.

2 The category $\mathcal{O}$ Projective functors in the category $\mathcal{O}$ are Frobenius. The Zuckerman functors are almost Frobenius.

If $V$ is a finite-dimensional representation of a Lie algebra $\mathfrak{g}$, let $T_{V}$ be the functor of tensoring with $V$ (this is a functor in the category of $U(\mathfrak{g})$-modules). It has a biadjoint functor $T_{V^{*}}$. Let $\mathfrak{g}$ be a finite-dimensional simple Lie algebra (over $\mathbb{C}$ ), $Z$ the center of $U(\mathfrak{g})$. Let $\mathcal{C}$ be the category of finitely-generated $U(\mathfrak{g})$-modules on which $Z$ acts through a finite-dimensional quotient. $\mathcal{C}$ decomposes into a direct sum of categories, one for each maximal ideal of $Z$. The category $\mathcal{C}_{\theta}$ associated to a maximal ideal $\theta$ consists of modules annihilated by some power of this ideal. Let $P_{\theta}: \mathcal{C} \rightarrow \mathcal{C}_{\theta}$ be the projection functor onto this direct summand. Then $T_{V}: \mathcal{C} \rightarrow \mathcal{C}$ decomposes into infinite direct $\operatorname{sum} \underset{\theta, \theta^{\prime}}{\oplus} P_{\theta^{\prime}} T_{V} P_{\theta}$. Each summand has a biadjoint functor $P_{\theta} T_{V^{*}} P_{\theta^{\prime}}$. Often these direct summands can be further decomposed into a direct sum. A direct summand of a functor $T_{V}: \mathcal{C} \rightarrow \mathcal{C}$ is a called a projective functor [9]. A projective functor is Frobenius.

The rather large category of $Z$-finite $U(\mathfrak{g})$-modules has a relatively small subcategory, often called the category $\mathcal{O}$. Let $\mathfrak{h} \subset \mathfrak{b}$ be a Cartan and Borel subalgebras of $\mathfrak{g}$. The category $\mathcal{O}$ of highest weight modules is a full subcategory of finitely-generated $U(\mathfrak{g})$-modules which consists of $\mathfrak{h}$-diagonalizable $U(\mathfrak{b})$ locally-finite modules [9].

Just like $\mathcal{C}$, the category $\mathcal{O}$ decomposes into an infinite direct sum of subcategories $\mathcal{O}_{\theta}$, over all maximal ideals $\theta$ of $Z$. $\mathcal{O}$ is stable under tensoring with a finite-dimensional module. Restrictions of projective functors to $\mathcal{O}$ also have biadjoints.

Let $\mathfrak{p} \supset \mathfrak{b}$ be a parabolic subalgebra and $\mathcal{O}_{\mathfrak{p}}$ the subcategory of $\mathcal{O}$ which consists of locally $U(\mathfrak{p})$-finite modules. The inclusion functor $I_{\mathfrak{p}}$ of $\mathcal{O}_{\mathfrak{p}}$ into $\mathcal{O}$ admits a left adjoint functor $Q_{\mathfrak{p}}$, which to a highest weight module assigns its maximal $U(\mathfrak{p})$-finite quotient, and a right adjoint functor $\Gamma_{\mathfrak{p}}$ which assigns to a module its maximal $U(\mathfrak{p})$-finite submodule.

$\Gamma_{\mathfrak{p}}$ and its right derived functor $R \Gamma_{\mathfrak{p}}$ are often called Zuckerman functors, while $Q_{\mathfrak{p}}$ and its left derived functor $L Q_{\mathfrak{p}}$ are sometimes called Bernstein functors. Functor isomorphisms

$$
R \Gamma_{\mathfrak{p}}[d] \cong L Q_{\mathfrak{p}}, \quad Q_{\mathfrak{p}} \cong R^{d} \Gamma_{\mathfrak{p}}
$$


tell us that in the derived category of $\mathcal{O}$ the left adjoint to $I_{\mathfrak{p}}$ is isomorphic to the right adjoint, up to the shift by $d$, where $d=\operatorname{dim}(\mathfrak{m})-\operatorname{dim}(\mathfrak{h})$ and $\mathfrak{m}$ is the Levi subalgebra of $\mathfrak{p}$. If the left adjoint of a functor is isomorphic to the right adjoint after a composition with an invertible functor, we say that the functor is almost Frobenius. In particular, the inclusion functor $I_{\mathfrak{p}}: D^{b}\left(\mathcal{O}_{\mathfrak{p}}\right) \rightarrow D^{b}(\mathcal{O})$ and the Zuckerman functor $R \Gamma_{\mathfrak{p}}$ are almost Frobenius.

Proposition 25, modified for decorated TQFT with corners, tells us that functors $F(M)$ are almost Frobenius, rather than just Frobenius. Therefore, categories associated to $(n-2)$-manifolds in $n$-dimensional decorated TQFTs with corners should have many Frobenius and/or almost Frobenius functors.

More examples of almost Frobenius functors:

- Functors $\mathcal{F}(b)$, for $b \in B_{n}^{m}$, as functors between categories $H^{n}$-mod and $H^{m}$-mod of graded modules. The left adjoint of $\mathcal{F}(b)$ is isomorphic to $\mathcal{F}(W(b))\{k-l\}$, the right adjoint is isomorphic to $\mathcal{F}(W(b))\{l-k\}$, where $l$ is the number of arcs connecting top endpoints of $b$ and $k$ is the number of arcs connecting bottom endpoints of $b$.

- Whenever we are working with graded symmetric algebras, the functor of the tensor product with a graded sweet bimodule will be almost Frobenius (as a functor between categories of graded modules).

- Same for differential graded symmetric algebras.

3 Coherent sheaves on Calabi-Yau manifolds Let $X$ and $Y$ be smooth complex projective varieties. Denote by $D(X)$ the bounded derived category of the abelian category of coherent sheaves on $X$.

Convolution with a complex $K$ of coherent sheaves on $D(X \times Y)$ is a functor $C_{K}$ from $D(X)$ to $D(Y)$. This functor takes a complex of sheaves on $X$, pulls it back to $X \times Y$, tensors by $K$, and pushes forward to $Y$ (all pulls, pushes and tensorings are derived). The left and right adjoint functors to $C_{K}$ are convolutions with $K^{*} \otimes \pi_{X}^{*} \omega_{X}[\operatorname{dim} X]$ and $K^{*} \otimes \pi_{Y}^{*} \omega_{Y}[\operatorname{dim} Y]$, where $K^{*}$ is the dual of $K, \pi_{X}, \pi_{Y}$ are projections from $X \times Y$ onto its factors, and $\omega_{X}, \omega_{Y}$ are canonical line bundles on $X$ and $Y$ (see [14],[10] and references therein).

If the line bundles $\pi_{X}^{*} \omega_{X}$ and $\pi_{Y}^{*} \omega_{Y}$ are trivial when restricted to the support of sheaf $K$, the functor of convolution with $K$ will have isomorphic (up to shift by $\operatorname{dim} X-\operatorname{dim} Y$ in the derived category) left and right adjoint functors. In particular, if $X, Y$ are Calabi-Yau varieties, so that $\omega_{X}, \omega_{Y}$ are trivial, then convolution with any complex of sheaves $K$ on $X \times Y$ has isomorphic (up to a shift) left and right adjoint functors. That's plenty of almost Frobenius functors to choose from. 
4 Fukaya-Floer categories It is expected that for a symplectic manifold $M$, subject to suitable conditions, the Fukaya $A_{\infty}$-category of lagrangian submanifolds in $M$ can be made into an $A_{\infty}$-triangulated category $F(M)$ (see $[41],[24],[25])$.

Convolution with a lagrangian submanifold $L$ in the direct product $M \times N$ of symplectic manifolds will define a pair of $A_{\infty}$-functors $F(M) \rightarrow F(N), F(N) \rightarrow$ $F(M)$. These $A_{\infty}$-functors will be biadjoint, up to shifts in the grading.

When $M$ and $N$ are symplectic Calabi-Yau manifolds, these examples of almost biadjoint functors will be mirror dual to functors of convolution with bounded complexes of coherent sheaves on the direct product of algebraic Calabi-Yau varieties.

5 Convolutions with smooth sheaves Let $f: Y \rightarrow X$ be a continuous map of good topological spaces, and $k$ a field. Consider the categories of sheaves of $k$-vector spaces on $Y$ and $X$ and their derived categories $D^{b}(Y)$ and $D^{b}(X)$. In the following discussion all functors are assumed derived.

The direct image functor $f_{*}: D^{b}(Y) \rightarrow D^{b}(X)$ has a left adjoint functor $f^{*}$, and the direct image with proper supports $f$ ! has a right adjoint functor $f^{\text {! }}$ (see [28], for instance). If $f$ is proper then $f_{*} \cong f_{!}$, so that $f_{*}$ has left and right adjoint functors. Further assume that $f$ is a locally-trivial fibration with a fiber-smooth closed orientable manifold $U$ of dimension $n$, and that the fibration is orientable, i.e. fibers $U_{x}$ can be oriented in a compatible way as $x$ varies over $X$. Then

$$
f^{!} \cong f^{*}[n],
$$

and $f_{*}$ is an almost Frobenius functor.

In interesting examples $Y$ is fibered over $X$ in two different ways and we get a Frobenius functor in the category $D^{b}(Y)$. For instance, Let $X$ be the variety of full flags in $\mathbb{C}^{n}, X_{i}$ variety of partial flags with the subspace of dimension $i$ omitted from the flag, and $Y_{i}=X \times_{X_{i}} X$. Then $Y_{i}$ is a locally-trivial $\mathbb{P}^{1}$ fibration over $X$ in two ways, $X \stackrel{f_{1}}{\longleftarrow} Y_{i} \stackrel{f_{2}}{\longrightarrow} X$, and defines a convolution functor $f_{2 *} f_{1}^{*}$ in $D^{b}(X)$. This functor is Frobenius, with a biadjoint $f_{1 *} f_{2}^{*}[2]$.

The localization theorem of Beilinson-Bernstein implies that this example of Frobenius and almost Frobenius functors is essentially equivalent to the Zuckerman functors example discussed earlier.

We see that derived categories of modules over symmetric algebras, of category $\mathcal{O}$, of coherent sheaves on Calabi-Yau varieties and Fukaya-Floer categories 
admit many biadjoint functors. In Section 6.5 we point out that quite often these categories have natural braid group actions, easily passing our test: to have a lot of invertible and biadjoint functors. What remains to be done is the much harder work of sifting through the universe of Frobenius algebras and Calabi-Yau varieties to find the precious ones that provide invariants of link cobordisms (we believe that Frobenius rings $H^{n}$ constitute the first nontrivial example). This problem will be addressed elsewhere.

\subsection{D TQFT with corners}

Let $R$ be a commutative ring. A 2-dimensional topological quantum field theory over $R$ is a tensor functor from the category $\mathcal{M}$ of 2-cobordisms between 1 manifolds to the category of $R$-modules. 1-manifolds are assumed oriented, compact and closed, cobordisms are oriented, compact 2-manifolds. 2D TQFTs over $R$ are in a bijection with commutative Frobenius algebras over $R$ (see [1], $[6$, Section 4.3]).

Any commutative Frobenius algebra is symmetric, so that there is a chain of inclusions of sets:

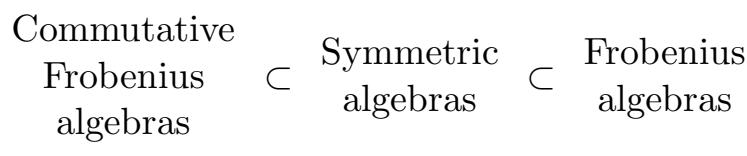

A commutative Frobenius $R$-algebra $A$ defines a 2D TQFT

$$
F_{A}: \mathcal{M} \longrightarrow R \text {-mod }
$$

that associates $A^{\otimes n}$ (the tensor product is over $R$ ) to a 1-manifold diffeomorphic to $n$ circles and maps

$$
m_{A}: A \otimes A \longrightarrow A, \quad \iota_{A}: R \longrightarrow A, \quad \operatorname{Tr}: A \longrightarrow R
$$

to the cobordisms depicted in Figure 2.

Examples of commutative Frobenius algebras are:

(1) The algebra $H^{\text {even }}(M, R)$ of even-dimensional cohomology groups of an even-dimensional closed oriented manifold $M$, where $R$ is a field.

(2) The local algebra of a finite-multiplicity holomorphic map $\mathbb{C}^{n} \rightarrow \mathbb{C}^{n}[3$, Section 5.11].

(3) Finite direct sums and tensor products of commutative Frobenius $R$ algebras. 
(4) The trivial extension algebra [26] of a finite-dimensional commutative algebra.

A 2D TQFT with corners over $R$ associates

- an additive $R$-linear category $F(K)$ to an oriented 0-manifold $K$,

- an $R$-linear functor $F(M): F\left(\partial_{0} M\right) \longrightarrow F\left(\partial_{1} M\right)$ to a 1-dimensional oriented cobordism $M$,

- a natural transformation $F(N): F\left(\partial_{0} N\right) \longrightarrow F\left(\partial_{1} N\right)$ to a 2-dimensional oriented cobordism $N$.

Surprisingly, we were unable to find any examples of 2D TQFTs with corners in the literature, and decided to construct some here, especially since they turned out to be remarkably similar to the 2-functor, described in Section 2.9, from the 2-category of surfaces in $\mathbb{R}^{3}$ to the 2-category of bimodules and bimodule maps.

We will build a restricted 2D TQFT with corners from a commutative Frobenius $R$-algebra $A$. The specialization of this TQFT with corners to closed 1-manifolds and cobordisms between them is the TQFT $F_{A}$ mentioned above.

An oriented 0-manifold consists of several points with orientations, that is, several points with plus and minus signs assigned to them. To simplify, we consider only 0 -manifolds with the same number of plus and minus points. Any such manifold is diffeomorphic to $2 n$ points, of which $n$ are plus points and $n$ are minus. We fix one manifold for each $n$ and denote it by $\bar{n}$. In our figures we'll always place the plus points to the left of the minus points.

We will use the same rule as the one for oriented tangles (Section 3.1) to induce orientations on the boundaries of an oriented 1-cobordism. Figure 43 is a diagram of a $(\overline{2}, \overline{3})$-cobordism (intersections should be ignored).

A 1-cobordism from $\bar{n}$ to $\bar{m}$ will also be called a $(\bar{m}, \bar{n})$-cobordism. We call basic a 1-cobordism which does not contain circles. There are $(n+m)$ ! basic $(\bar{m}, \bar{n})$-cobordisms.

Denote by $\widehat{\mathrm{Cob}}_{n}^{m}$ the set of $(\bar{m}, \bar{n})$-cobordisms, by $\mathrm{Cob}_{n}^{m}$ the set of basic $(\bar{m}, \bar{n})$ cobordisms, and by $\mathrm{Cob}^{n}$ the set of basic $(\bar{n}, \overline{0})$-cobordisms. Let $W$ be the involution $W: \widehat{\operatorname{Cob}}_{n}^{m} \longrightarrow{\widehat{\mathrm{Cob}_{m}}}_{m}^{n}$ that turns a 1-cobordism upside-down and changes all orientations to make cobordisms $W(b)$ and $b$ composable, as depicted in Figure 44.

Let Vert $\bar{n}$ be the identity 1-cobordism from $\bar{n}$ to $\bar{n}$, and $S(b)$, for $b \in \mathrm{Cob}^{n}$, the standard "contraction" 2-cobordism from $b W(b)$ to $\operatorname{Vert}_{\bar{n}}$. 


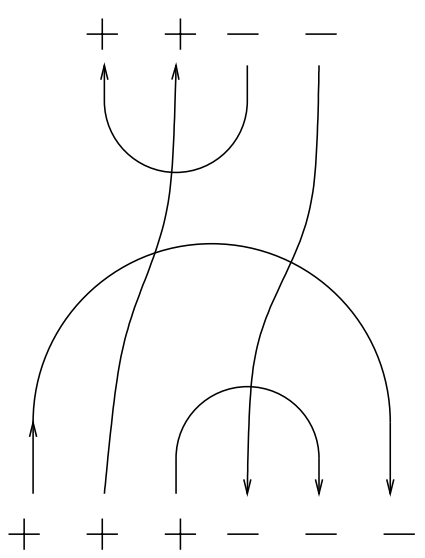

Figure 43: A 1-cobordism from $\overline{3}$ to $\overline{2}$
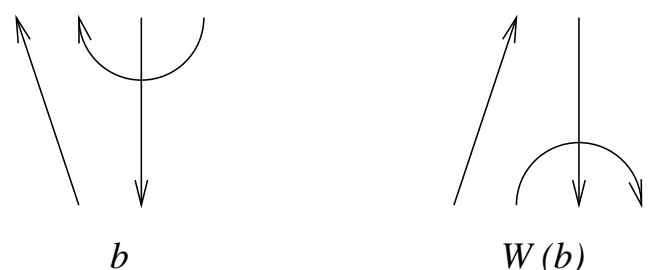

Figure 44: Involution $W$

These notations mimic the ones from Section 2, and the analogy is nearly complete. Instead of flat tangles we are looking at oriented 1-cobordisms, instead of surfaces in $\mathbb{R}^{3}$ we are looking at oriented 2-manifolds with corners, considered as cobordisms between 1-manifolds with boundary. We denote by $\mathbb{M}$ our 2 -category of oriented 2-cobordisms with corners.

For each $n \geq 0$ define an $R$-algebra $A^{n}$ by

$$
A^{n} \stackrel{\text { def }}{=} \underset{a, b \in \operatorname{Cob}^{n}}{\oplus} b\left(A^{n}\right)_{a}, \quad b\left(A^{n}\right)_{a} \stackrel{\text { def }}{=} F_{A}(W(b) a) .
$$

$W(b) a$ is a closed 1-manifold, and we can apply the functor $F_{A}$ to it. The multiplication in $A^{n}$ is induced by 2-cobordisms $\operatorname{Id}_{W(c)} S(b) \operatorname{Id}_{a}$ from $W(c) b W(b) a$ to $W(c) a$ :

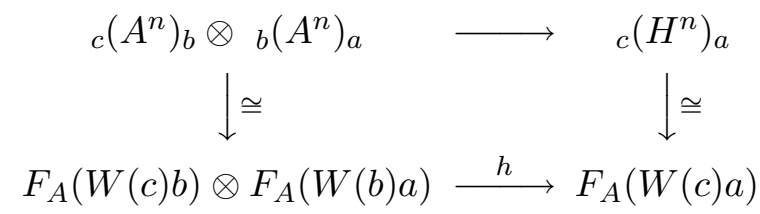

Algebraic 83 Geometric Topology, Volume 2 (2002) 
Here $h=F_{A}\left(\operatorname{Id}_{W(c)} S(b) \operatorname{Id}_{a}\right)$.

To a 1-cobordism $a \in{\widehat{\mathrm{Cob}_{n}}}_{n}^{m}$ we associate an $\left(A^{m}, A^{n}\right)$-bimodule $F_{A}(a)$ by

$$
F_{A}(a) \stackrel{\text { def }}{=}{ }_{b \in \mathrm{Cob}^{n}, c \in \mathrm{Cob}^{m}}^{\oplus} c F_{A}(a)_{b}, \quad{ }_{c} F_{A}(a)_{b} \stackrel{\text { def }}{=} F_{A}(W(c) a b),
$$

the bimodule structure is defined analogously to the one in Section 2.7. All results and constructions of Section 2.7 have their counterparts:

Proposition 27 (1) For a 1-cobordism $a \in \widehat{C}_{n}^{m}$ the bimodule $F_{A}(a)$ is a sweet $\left(A^{m}, A^{n}\right)$-bimodule.

(2) A 2-cobordism $S$ induces a homomorphism of bimodules

$$
F_{A}\left(\partial_{0} S\right) \longrightarrow F_{A}\left(\partial_{1} S\right) \text {. }
$$

The homomorphism assigned to the composition of 2-cobordisms equals the composition of homomorphisms.

(3) For 1-cobordisms $a \in{\widehat{\mathrm{Cob}_{n}}}_{n}^{m}$ and $b \in{\widehat{\mathrm{Cob}_{m}}}^{k}$ bimodules $F_{A}(b) \otimes_{A^{m}} F_{A}(a)$ and $F_{A}(b a)$ are canonically isomorphic.

These results amount to:

Proposition $28 \quad F_{A}$ is a 2-functor from the 2-category $\mathbb{M}$ of oriented 2cobordisms with corners to the 2-category $\mathbb{B}_{A}$ of sweet $A^{n}, n \geq 0$ bimodules and bimodule homomorphisms.

Here $\mathbb{B}_{A}$ is a 2-category with objects-nonnegative integers, 1-morphisms from $n$ to $m$-sweet $\left(A^{m}, A^{n}\right)$-bimodules and 2-morphisms-homomorphisms of bimodules. We call $\mathbb{B}_{A}$ the 2-category of sweet $A$-bimodules.

$F_{A}$ is a restricted 2D TQFT with corners that to the 0 -manifold $\bar{n}$ associates the category of left $A^{n}$-modules, to a 1-cobordism $a$ the functor of tensor product with the bimodule $F_{A}(a)$, and to a 2-cobordism $S$ the natural transformation of functors induced by the bimodule homomorphism (36).

As a special case of Proposition 25 we obtain:

Proposition 29 For a 1-cobordism $b \in{\widehat{\mathrm{Cob}_{n}}}^{m}$ the functor $F_{A}(W(b))$ is left and right adjoint to the functor $F_{A}(b)$.

$A^{n}$ has a nondegenerate symmetric trace $\operatorname{Tr}_{n}: A^{n} \rightarrow R$ defined by $\operatorname{Tr}_{n}(x)=0$ if $x \in{ }_{b}\left(A^{n}\right)_{a}$ and $b \neq a$, and $\operatorname{Tr}_{n}(x)=\operatorname{Tr}^{\otimes n}(x)$ if $x \in{ }_{a}\left(A^{n}\right)_{a}$. Namely, ${ }_{a}\left(A^{n}\right)_{a} \cong A^{\otimes n}$ and we define the trace on ${ }_{a}\left(A^{n}\right)_{a}$ to be the tensor product of trace functions $\operatorname{Tr}: A \rightarrow R$. 
Proposition $30 A^{n}$ is a symmetric $R$-algebra.

The proof is similar to that of Proposition 32 .

This elementary construction of 2D TQFTs with corners warmly welcomes symmetric nonsemisimple algebras. Symmetric algebras can be thought of as tools for producing biadjoint functors. If $A=\mathcal{A}$, the ring introduced in Section 2.1, $A^{n}$ contains $H^{n}$ as a subring, so that the 2D TQFT with corners $F_{\mathcal{A}}$ carries in it the structure that categorifies the Kauffman bracket of tangles.

\subsection{Triangulated categories, mapping class groups, and four- dimensional topological quantum field theories}

An $n$-dimensional TQFT with corners associates a category to a closed oriented $(n-2)$-manifold $K$. The mapping class group of $K$, i.e., the group of connected components of the diffeomorphism group of $K$ must act on the category $F(K)$.

The only examples of 2D TQFT with corners that we know of are the ones described in Section 6.4. The oriented 0-manifold $\bar{m}$ is a union of $m$ positively oriented and $m$ negatively oriented points, the mapping class group $\operatorname{Map}(\bar{m})$ of $\bar{m}$ permutes these points preserving their orientations, and is isomorphic to the product $S_{m} \times S_{m}$ of two symmetric groups. Map $(\bar{m})$ acts naturally on the algebra $A^{m}$, and, therefore, on the category $A^{m}$-mod that the 2D TQFT $F_{A}$ associates to the 0 -manifold $\bar{m}$. There is nothing mysterious about his action.

When $n=3$ the category $F(K)$ is associated to a one-manifold $K$, a disjoint union of circles. The orientation-preserving mapping class group of a circle is trivial. The mapping class group of the union of $m$ circles is $S_{m}$. In the famous example of the Witten-Reshetikhin-Turaev TQFT the category $F\left(S^{1}\right)$ assigned to the circle is semisimple, with finite number of (isomorphism classes of) simple objects. The category assigned to the union of $m$ circles is the $m$-tensor power of $F\left(S^{1}\right)$, with the mapping class group acting by permutations. We see that in dimension 3 the mapping class group action on $F(K)$ is equally unremarkable.

A 4-dimensional TQFT with corners should associate an additive category $F(K)$ to an oriented closed surface $K$. The mapping class group $\operatorname{Map}(K)$ of $K$, a large and complicated group, must act on $F(K)$. It is hard to come up with interesting actions of mapping class groups on categories. For starters, we will argue that mapping class groups of surfaces do not appear naturally as automorphism groups of abelian categories. Abelian categories are primarily associated to algebraic or topological structures: to an algebra $A$ we associate 
the category of $A$-modules, to a topological space $X$ the category of sheaves $\operatorname{Sh}(X)$ on $X$, to a ringed space the category of sheaves of modules, etc. In each of these cases all or nearly all automorphisms of the abelian category come from symmetries of the original object: from automorphisms of the algebra $A$, homeomorphisms of the space $X$, etc.

These symmetry groups are unrelated to mapping class groups of surfaces. The group of automorphisms of an algebra is typically a mixture of a finite group and a connected algebraic group. The group of homeomorphisms or diffeomorphisms of a surface $K$ does indeed quotients onto the mapping class group $\operatorname{Map}(K)$ of $K$. However, if $K$ has genus greater than 1, this quotient map does not admit a section.

Our objection to abelian categories as candidates for $F(K)$, for a surface $K$, grows even stronger if these abelian categories are semisimple. If $k$ is a field and $C$ a semisimple $k$-linear category, a $k$-linear automorphism of $C$ can do nothing but permute simple objects. An action of the mapping class group of $K$ on $C$ amounts to a homomorphism to a symmetric group. Such simple action is unlikely to lead to a sophisticated $4 \mathrm{D}$ TQFT that we are searching for.

We believe that this informal argument destroys any hope of constructing 4dimensional TQFTs of Donaldson-Floer-Seiberg-Witten variety directly from abelian categories (the 4-dimensional relatives [44] of the 3-dimensional Dijkgraaf-Witten TQFT [19], built from a finite group, associate semisimple categories to surfaces, but these TQFTs are toy models).

Things appear much brighter when we consider instead derived categories of abelian categories, and, more generally, triangulated categories. A very strong positive indicator that triangulated categories are related to 4-dimensional TQFTs would be provided by a triangulated category $C$ with a faithful action of the mapping class group of a genus $g$ closed oriented surface, and such that the Grothendieck group of $C$ has finite rank. Examples are not known at present $^{2}$. Interesting examples are available, however, of derived categories with a faithful action of the braid group. These actions do not come from actions on the underlying abelian categories. We list several examples below.

I The first example of a braid group action on a derived category came up about 20 years ago, in the early days of the geometric representation theory, but until recently remained an unpublished folk theorem.

\footnotetext{
${ }^{2}$ Not counting cheats of the following kind: choose a faithful representation $V$ (if you can find one) of the mapping class group. There is a faithful action of the mapping class group on the exterior algebra $\Lambda V$ of $V$, therefore, on the category of $\Lambda V$-modules and on the derived category $D^{b}(\Lambda V$-mod).
} 
Let $G$ be a simply-connected simple complex Lie group and $B$ a Borel subgroup. For each simple root $\alpha$ the flag variety $X=G / B$ fibers over $G / P_{\alpha}$ with fiber $\mathbb{P}^{1}$, where $P_{\alpha} \supset B$ is the minimal parabolic subgroup associated to $\alpha$. Denote this fibration by $p_{\alpha}: X \rightarrow G / P_{\alpha}$. Let $Y_{\alpha}^{\prime} \subset X \times X$ be the subset $\left\{\left(x_{1}, x_{2}\right) \mid p_{\alpha}\left(x_{1}\right)=p_{\alpha}\left(x_{2}\right), x_{1} \neq x_{2}\right\}$ and $j_{\alpha}: Y_{\alpha}^{\prime} \hookrightarrow X \times X$ the inclusion. Let $\mathcal{F}_{\alpha}$ be the sheaf on $X \times X$ which is the direct image under $j_{\alpha}$ of the constant sheaf on $Y_{\alpha}^{\prime}$. Let $D^{b}(X)$ be the bounded derived category of sheaves of complex vector spaces on $X$. Let $R_{i}$ be the functor in $D^{b}(X)$ of convolution with $\mathcal{F}_{\alpha}$.

Denote by $B(G)$ the generalized braid group associated to the Dynkin diagram of $G$.

Proposition 31 Functors $R_{i}$ are invertible and generate an action of the braid group $B(G)$ on the category $D^{b}(X)$.

See [50] for a proof and [47] for a related discussion.

$D^{b}(X)$ is a very large category. Let $D$ be the full subcategory of $D^{b}(X)$ which consists of objects with finite-dimensional constructible cohomology relative to the stratification of $X$ by orbits of the left multiplication action of $B$ (the Schubert stratification). This is a "small" category, in the sense that its Grothendieck group has finite rank, and $D$ is isomorphic to the derived category of modules over a finite-dimensional algebra (and to the derived category of a regular block of the category $\mathcal{O}$ ). It is not hard to see that the above action of the braid group preserves $D$.

The following generalization of this action to actions on derived categories of sheaves on partial flag varieties seems to be new. For a sequence $\mathbf{n}=$ $\left(n_{1}, \ldots, n_{k}\right)$ of positive integers denote by $X(\mathbf{n})$ the variety of partial flags in $\mathbb{C}^{n}$, where $n=n_{1}+\cdots+n_{k}$ :

$$
X(\mathbf{n})=\left\{0=L_{0} \subset L_{1} \subset \cdots \subset L_{k-1} \subset L_{k}=\mathbb{C}^{n}, \operatorname{dim} L_{i}=n_{1}+\cdots+n_{i}\right\} .
$$

Denote by $s_{i} \mathbf{n}$ the sequence $\mathbf{n}$ with entries $n_{i}$ and $n_{i+1}$ transposed. Let $Y \subset$ $X(\mathbf{n}) \times X\left(s_{i} \mathbf{n}\right)$ be the subset

$$
\left\{\left(L_{0}, L_{1}, \ldots, L_{k}\right) \times\left(L_{0}, \ldots, L_{i-1}, L_{i}^{\prime}, L_{i+1}, \ldots, L_{k}\right), \quad L_{i} \cap L_{i}^{\prime}=L_{i-1}\right\} .
$$

In other words, the two partial flags coincide except at the $i$-th term while $L_{i}, L_{i}^{\prime}$ are in general position.

Consider the sheaf on $X(\mathbf{n}) \times X\left(s_{i} \mathbf{n}\right)$ which is the direct image of the constant sheaf on $Y$ under the inclusion $Y \subset X(\mathbf{n}) \times X\left(s_{i} \mathbf{n}\right)$. Convolution with this sheaf is an invertible functor between derived categories of sheaves on $X(\mathbf{n})$ 
and $X\left(s_{i} \mathbf{n}\right)$ (for real flag varieties this is Exercise III.15 in [33]). Denote by $\widetilde{X}$ the disjoint union of $X(\mathbf{m})$, over all possible permutations $\mathbf{m}$ of $\mathbf{n}$. For each $i, 1 \leq i \leq k-1$, we get a functor $R_{i}$ in the derived category of sheaves on $\widetilde{X}$. These functors generate a braid group action.

Let $\mathcal{O}_{r}$ be a regular block of the category $\mathcal{O}$ for $\mathfrak{s l}_{n}$ and $\Theta_{i}: \mathcal{O}_{r} \rightarrow \mathcal{O}_{r}$ translation across the $i$-th wall functor. $\Theta_{i}$ is the composition of two biadjoint functors, translations on and off the $i$-th wall, and there is a natural transformation $\Theta_{i} \longrightarrow$ Id. Denote by $\sigma_{i}^{\prime}$ the functor in the derived category $D^{b}\left(\mathcal{O}_{r}\right)$ which is the cone of this morphism of functors. Let $\Gamma_{i}$ be the Zuckerman functor of taking the maximal $U\left(\mathfrak{p}_{i}\right)$-locally finite submodule, where $\mathfrak{p}_{i} \supset \mathfrak{b}$ is the $i$-th minimal parabolic subalgebra. There is a morphism of functors $\Gamma_{i} \longrightarrow \mathrm{Id}$, which is just the inclusion of the submodule into the module. The cone of the induced morphism of derived functors $R \Gamma_{i} \longrightarrow \mathrm{Id}$ is a functor in $D^{b}\left(\mathcal{O}_{r}\right)$, which we denote by $\sigma_{i}^{\prime \prime}$. Functors $\sigma_{i}^{\prime}, \sigma_{i}^{\prime \prime}, 1 \leq i \leq n-1$ generate two commuting braid group actions in $D^{b}\left(\mathcal{O}_{r}\right)$.

II A $2 n$-string braid $\sigma$ is an $(n, n)$-tangle, so that to $\sigma$ we can associate the complex $\mathcal{F}(\sigma)$ of sweet $\left(H^{n}, H^{n}\right)$-bimodules (Theorem 2). The tensor product with this complex is an invertible functor in the category $\mathcal{K}_{P}^{n}$ of complexes of projective $H^{n}$-modules, as well as in the derived category of $H^{n}$-modules. We see that these categories admit a highly nontrivial braid group action.

III A simpler example of braid group actions was found in [52] and [39] and later considered in [53] and [27]. To a finite graph $\Gamma$ one associates a finitedimensional algebra $A(\Gamma)$, the quadratic dual of the Gelfand-Ponomarev algebra of $\Gamma$ (see [27]). The braid group associated to the graph $\Gamma$ acts in the derived category of the category of $A(\Gamma)$-modules. It is proved in [39] that when $\Gamma$ is the chain of length $n$ the braid group of $\Gamma$ (isomorphic to the $(n+1)$-stranded braid group) acts faithfully in the derived category $D^{b}(A(\Gamma)$-mod).

IV Suppose that there is an action of a group $H$ in the derived category of modules over an algebra $A$, and that the action is given explicitly: there is an invertible functor $F_{g}$, for each $g \in H$, of tensoring with a bounded complex $C(g)$ of $A$-bimodules which are right $A$-projective, and there are homotopy equivalences of complexes $C(g) \otimes_{A} C(h) \cong C(g h)$ of $A$-bimodules for any $g, h \in$ $H$. Let $A^{(n)}$ be the cross-product of $A^{\otimes n}$ and the group ring of the symmetric group $S_{n}$ :

$$
A^{(n)} \stackrel{\text { def }}{=} A^{\otimes n} \otimes_{\mathbb{Z}} \mathbb{Z}\left[S_{n}\right], \quad\left(a_{1} \otimes s_{1}\right)\left(a_{2} \otimes s_{2}\right)=a_{1} s_{1}\left(a_{2}\right) \otimes s_{1} s_{2},
$$


where $a_{i} \in A^{\otimes n}, s_{i} \in S_{n}$ and $S_{n}$ acts on $A^{\otimes n}$ by permutations. The complex $C(g)$ gives rise to the complex

$$
C(g) \stackrel{(n) \stackrel{\text { def }}{=}}{=} C(g)^{\otimes n} \otimes_{\mathbb{Z}} \mathbb{Z}\left[S_{n}\right]
$$

of $A^{(n)}$-bimodules, and there are homotopy equivalences

$$
C(g)^{(n)} \otimes_{A^{(n)}} C(h)^{(n)} \cong C(g h)^{(n)} .
$$

We obtain an action of $H$ in the derived category of $A^{(n)}$-modules.

The cross-product algebra $A^{(n)}$ can be viewed as the $n$-th symmetric power of $A$, and categories $A^{(n)}$-mod and $D^{b}\left(A^{(n)}\right.$-mod) as $n$-th symmetric powers of categories $A$-mod and $D^{b}(A$-mod). Then our informal rule is:

A group action on a category gives rise to actions on all symmetric powers of the category.

This can also be applied to group actions in the derived categories of sheaves. If $H$ acts explicitly on the derived category $D(X)$ of sheaves on $X$, via convolutions with complexes of sheaves $C(g)$ on $X \times X$, then $H$ also acts in the derived categories of $S_{n}$-equivariant sheaves on $X^{\times n}$, for all $n$. Here $X$ could be a manifold, a stratified space, a scheme, and $D(X)$ the derived category of sheaves, or the category of cohomologically constructible complexes of sheaves, or the derived category of coherent sheaves on $X$.

It is particularly interesting to apply this construction to the action in example III of the affine braid group $B(\Gamma)$ in the derived category of $A(\Gamma)$-modules, for an affine simply-laced Dynkin diagram $\Gamma$. The algebra $A(\Gamma)$ is Morita equivalent to the cross-product $\Lambda(G)$ of the exterior algebra on 2 generators and the group algebra $G$ of the finite subroup of $S U(2)$ associated to $\Gamma$ via the McKay correspondence [27]. In turn, the Koszul dual $S(G)$ of $\Lambda(G)$ is the cross-product of the polynomial algebra on 2 generators and the group algebra of $G$. The derived category of finitely-generated $S(G)$-modules is equivalent to the derived category of coherent sheaves on the minimal resolution $X(G)=\widetilde{\mathbb{C}^{2} / G}$ of a simple singularity $\mathbb{C}^{2} / G[32]$.

The action of the affine braid group $B(\Gamma)$ in the derived category of $A(\Gamma)$ modules induces, through these derived equivalences, an action in the derived category of coherent sheaves on $X(G)$, and, therefore, in the derived category of $S_{n}$-equivariant coherent sheaves on $X(G)^{\times n}$.

At the same time, we get affine braid group actions in the derived categories of modules over cross-product algebras $A(\Gamma)^{(n)}, \Lambda(G)^{(n)}$, and $S(G)^{(n)}$. 
Algebras $S(G)^{(n)}$ can be viewed as cross-products of a polynomial algebra on $2 n$ generators and group algebras of finite groups $G_{n}$, where $G_{n}$ is the crossproduct of $G^{\times n}$ and $S_{n}$. Group algebras of $G_{n}$ appeared in [22] (Warning: Their $\Gamma_{n}$ is our $G_{n}$, while we use $\Gamma$ to denote an affine Dynkin diagram), algebras $S(G)^{(n)}$ appeared in [56]. Weiqiang Wang [56] conjectured that categories of coherent sheaves on the Hilbert scheme of $n$-points on $X(G)$ and of finitely-generated modules over $S(G)^{(n)}$ are derived equivalent. If true, this would imply that our action of the affine braid group in the derived category of coherent sheaves on $X(G)$ gives rise to actions in the derived categories of coherent sheaves on Hilbert schemes of $X(G)$. Similar braid group actions should exist in the derived categories of coherent sheaves on Nakajima quiver varieties, lifting the known action [46, Section 9] of Weyl groups on cohomology groups of quiver varieties.

In addition, we expect that a derived equivalence between categories of coherent sheaves on two algebraic surfaces induces a derived equivalence between categories of coherent sheaves on Hilbert schemes of these surfaces.

V Rouquier conjectured [51] that there are braid group actions in derived categories of regular blocks of representations of algebraic groups and of modules over group algebras of symmetric groups over fields of finite characteristic.

This abundance of braid group actions enhances our beliefs that triangulated and derived categories are the right place to search for 4-dimensional TQFTs, and that quantum invariants of link cobordisms and surfaces in $\mathbb{R}^{4}$ hide in derived categories of the category $\mathcal{O}$, categories of modules over Frobenius algebras, and categories of coherent sheaves on Nakajima varieties.

\subsection{Commutative Frobenius algebras and the Temperley-Lieb 2-category}

All constructions and results of Sections 2.1,2.4-2.9 admit a straightforward generalization from the ring $\mathcal{A}$ to an arbitrary commutative Frobenius $R$ algebra $A$.

As outlined in Section 6.4, $A$ gives rise to a 2-dimensional TQFT $F_{A}$. Instead of the ring $H^{n}$ consider the $R$-algebra $H_{A}^{n}$ :

$$
H_{A}^{n}=\underset{a, b \in B^{n}}{\oplus} F_{A}(W(b) a)
$$

with the multiplication defined via elementary cobordisms in the same manner as for $H^{n}$. 
To a 1-morphism $a \in \widehat{B}_{n}^{m}$ from $n$ to $m$ in the Temperley-Lieb 2-category we associate an $\left(H_{A}^{n}, H_{A}^{m}\right)$-bimodule

$$
F_{A}(a)=\underset{c \in B^{m}, b \in B^{n}}{\oplus} F_{A}(W(c) a b)
$$

and to 2-morphisms associate bimodule maps. In this way we get a 2-functor from the Temperley-Lieb 2-category $\mathbb{T L}$ to the 2-category of $\left(H_{A}^{m}, H_{A}^{n}\right)$-bimodules, over all $n, m \geq 0$.

Constructions and results of Sections 3 and 4, however, do not admit any easy extensions from $\mathcal{A}$ to other commutative Frobenius algebras (except for the algebra $A$ in [38] and anything obtained by base change from $A$ ).

\section{7 $\quad H^{n}$ is a symmetric ring}

Let $\operatorname{Tr}: H^{n} \rightarrow \mathbb{Z}$ be the $\mathbb{Z}$-linear map given by

- $\operatorname{Tr}(x)=0$ if $x \in{ }_{a}\left(H^{n}\right)_{b}$ and $a \neq b$,

- on ${ }_{a}\left(H^{n}\right)_{a}=\mathcal{A}^{\otimes n}$ the trace map is defined as $\epsilon^{\otimes n}: \mathcal{A}^{\otimes n} \rightarrow \mathbb{Z}$.

This is a symmetric functional, $\operatorname{Tr}(x y)=\operatorname{Tr}(y x)$, and we'll prove below that Tr is nondegenerate, that is, it makes $H^{n}$ into a symmetric ring:

Proposition $32 H^{n}$ is a symmetric ring.

Proof Notice that $H^{n}$ is a free abelian group. We'll find a basis $I$ of $H^{n}$ and an involution $*$ of $I$ such that for $x, y \in I$

$$
\operatorname{Tr}\left(x x^{*}\right)=1, \quad \operatorname{Tr}(x y)=0 \text { if } y \neq x^{*} .
$$

That would imply symmetricity of $H^{n}$.

Define $I$ as the union of bases ${ }_{a} I_{b}$ of ${ }_{a}\left(H^{n}\right)_{b}$, over all $a$ and $b$. We have ${ }_{a}\left(H^{n}\right)_{b} \cong$ $\mathcal{A}^{\otimes m}$ where $m$ is the number of circles in the closed diagram $W(a) b$. Define ${ }_{a} I_{b}$ as the product basis in $\mathcal{A}^{\otimes m}$, its elements are products of $1, X \in \mathcal{A}$.

The involution $*$ will take an element of ${ }_{a} I_{b}$ to an element of ${ }_{b} I_{a}$. To define $*$ notice that there is a natural contraction cobordism between $W(b) a W(a) b$ and the empty diagram. Since

$$
\mathcal{F}(W(b) a W(a) b) \cong \mathcal{F}(W(b) a) \otimes \mathcal{F}(W(a) b) \cong{ }_{b}\left(H^{n}\right)_{a} \otimes{ }_{a}\left(H^{n}\right)_{b},
$$

this cobordisms defines a nondegenerate bilinear pairing

$$
{ }_{b}\left(H^{n}\right)_{a} \otimes{ }_{a}\left(H^{n}\right)_{b} \longrightarrow \mathbb{Z} .
$$


The pairing, in fact, is the restriction of

$$
H^{n} \otimes H^{n} \stackrel{m}{\longrightarrow} H^{n} \stackrel{\operatorname{Tr}}{\longrightarrow} \mathbb{Z}
$$

to ${ }_{b}\left(H^{n}\right)_{a} \otimes{ }_{a}\left(H^{n}\right)_{b} \subset H^{n} \times H^{n}$.

The basis ${ }_{b} I_{a}$ is dual to ${ }_{a} I_{b}$ relative to the pairing (37). If $x \in{ }_{a} I_{b}$, a product of 1 's and $X$ 's, then define $x^{*}$ as the opposed product, namely, we substitute $X$ for $\mathbf{1}$ and $\mathbf{1}$ for $X$ in the product for $x$, and treat this product as an element of ${ }_{b}\left(H^{n}\right)_{a} \cong \mathcal{A}^{\otimes m} \cong{ }_{a}\left(H^{n}\right)_{b}$.

Proposition 33 The ring $H^{n}$ has infinite homological dimension if $n>0$.

Proof The abelian group $\operatorname{Hom}\left(P_{a}, P_{b}\right)$ (where we consider all homomorphisms, not only the grading-preserving ones) has even rank for any indecomposable projectives $P_{a}, P_{b}$. Therefore, an $H^{n}$-module isomorphic, as an abelian group, to $\mathbb{Z}$, does not admit a finite length projective resolution.

Of course, $H^{0}=\mathbb{Z}$ and $\mathbb{Z}$ has homological dimension 1 .

To conclude this section, we would like to point out the relation of the ring $H^{n}$ to meander determinants of Francesco, Golinelli and Guitter [21]. Let $k$ be a characteristic 0 field. Until the end of this section we denote the $k$-algebra $H^{n} \otimes_{\mathbb{Z}} k$ by $H_{k}^{n}$, and by $H_{k}^{n}$-mod the category of finite-dimensional left $H_{k}^{n}$ modules. Unlike previous sections, we consider modules which are not graded. As before, we denote by $P_{a}, a \in B^{n}$ indecomposable projective modules.

Let $G$ be the Grothendieck group of $H_{k}^{n}$-mod and $G^{\prime}$ its subgroup generated by projective modules. $G^{\prime}$ is a proper subgroup of $G$, since $\operatorname{dim} \operatorname{Hom}\left(P_{a}, P_{b}\right)$ is even for any $a, b$.

The Cartan matrix $C$ of $H_{k}^{n}$ is the $B^{n} \times B^{n}$-matrix with entries

$$
c_{a b}=\operatorname{dim} \operatorname{Hom}\left(P_{a}, P_{b}\right) .
$$

Notice that every entry is a power of 2 . This matrix is the $q=1$ specialization of the meander matrix in [21]. Its determinant was computed in [21] and equals

$$
\prod_{i=1}^{n}(i+1)^{c_{n, i}}, \quad c_{n, i}=\left(\begin{array}{c}
2 n \\
n-i
\end{array}\right)-2\left(\begin{array}{c}
2 n \\
n-i-1
\end{array}\right)+\left(\begin{array}{c}
2 n \\
n-i-2
\end{array}\right)
$$

where the convention is that $\left(\begin{array}{l}k \\ j\end{array}\right)=0$ if $j<0$.

In particular, the determinant is not 0 , which implies that $G^{\prime}$ is a finite index subgroup of $G$, and the index is given by the product in (38). 
$H_{k}^{n}$ is a symmetric and, therefore, a Frobenius $k$-algebra. We can form the stable category $H_{k}^{n}$ - $\underline{\bmod }$, the quotient of $H_{k}^{n}$-mod by morphisms which factor through a projective module. This is a triangulated category [26] with the Grothendieck group isomorphic to $G / G^{\prime}$. Thus, the meander determinant acquires a strange homological interpretation as the order of the Grothendieck group of $H_{k}^{n}$ - $\underline{\text { mod. }}$.

To each diagram $D$ of an $(m, n)$-tangle $L$ we associated a complex $\mathcal{F}(D)$ of sweet $(m, n)$-bimodules. This complex defines a functor between stable categories $H_{k}^{n}-\underline{\bmod }$ and $H_{k}^{m}-\underline{\bmod }$, and induces an action (in the weak sense, i.e. via isomorphism classes of functors) of the category of tangles on stable categories of $H_{k}^{n}$-modules.

\section{References}

[1] L. Abrams. Two-dimensional topological quantum field theories and Frobenius algebras. J. Knot Theory and its Ramifications, 5:569-589, 1996.

[2] S. Ariki. On the decomposition numbers of the Hecke algebra of $G(m, 1, n) . J$. Math. Kyoto Univ., 36(4):789-808, 1996.

[3] V. I. Arnold, S. M. Gusein-Zade, and A. N. Varchenko. Singularities of differentiable maps, vol.I. Monographs in Mathematics, 82. Burkhäuser, Boston, 1985.

[4] M. F. Atiyah. Topological quantum field theories. I.H.E.S. Publ. Math., 68:175$186,1988$.

[5] J. C. Baez and J. Dolan. Higher-dimensional algebra and topological quantum field theory. J. Math. Phys., 36(11):6073-6105, 1995.

[6] B. Bakalov and A. A. Kirillov, Jr. Lectures on tensor categories and modular functors. University Lecture Series 21. AMS, Providence, RI, 2001. A preliminary version is available at http://www.math.sunysb.edu/ ${ }^{\sim}$ kirillov.

[7] A. J. Berkson. The u-algebra of a restricted Lie algebra is Frobenius. Proc. Amer. Math. Soc., 15:14-15, 1964.

[8] J. Bernstein, I. B. Frenkel, and M. Khovanov. A categorification of the Temperley-Lieb algebra and Schur quotients of $U\left(\mathfrak{s l}_{2}\right)$ via projective and Zuckerman functors. Selecta Math., New Ser., 5:199-241, 1999. arXiv:math.QA/0002087

[9] J. Bernstein and S. Gelfand. Tensor products of finite- and infinite-dimensional representations of semisimple Lie algebras. Compositio Math., 41(2):245-285, 1980.

[10] A. Bondal and D. Orlov. Semiorthogonal decompositions for algebraic varieties. arXiv:alg-geom/9506012 
[11] P. J. Braam and S. K. Donaldson. Floer's work on instanton homology, knots and surgery. In The Floer memorial volume, Progr. Math., 133, pages 195-256. Birkhäuser, Basel, 1995.

[12] T. Braden. Perverse sheaves on Grassmannians. arXiv:math.AG/9907152.

[13] T. Braden and M. Khovanov. In preparation.

[14] T. Bridgeland. Equivalences of triangulated categories and Fourier-Mukai transforms. Bull. London Math. Soc., 31:25-34, 1999. arXiv:math.AG/9809114

[15] J. S. Carter, D. E. Flath, and M. Saito. The classical and quantum 6j-symbols. Mathematical Notes, 43. Princeton University Press, Princeton, NJ, 1995.

[16] J. S. Carter, L. H. Kauffman, and M. Saito. Diagrammatics, singularities and their algebraic interpretations. In 10th Brazilian Topology Meeting (São Carlos, 1996), Mat. Contemp. 13, pages 21-115, 1997.

[17] R. W. Carter. Representation theory of the 0-Hecke algebra. Journal of algebra, 104:89-103, 1986.

[18] L. Crane and I. B. Frenkel. Four-dimensional topological quantum field theory, Hopf categories, and the canonical bases. Journal of Mathematical Physics, 35(10):5136-5154, 1994.

[19] R. Dijkgraaf and E. Witten. Topological gauge theories and group cohomology. Comm. Math. Phys., 129(2):393-429, 1990.

[20] R. Farnsteiner and H. Strade. Modular Lie algebras and their representations. Monographs and textbooks in Pure and Applied math, 116. 1988.

[21] P. D. Francesco, O. Golinelli, and E. Guitter. Meanders and the Temperley-Lieb algebra. Comm. Math. Phys., 186:1-59, 1997.

[22] I. B. Frenkel, N. Jing, and W. Wang. Vertex representations via finite groups and the Mckay correspondence. arXiv:math.QA/9907166

[23] I. B. Frenkel and M. Khovanov. Canonical bases in tensor products and graphical calculus for $U_{q}\left(\mathfrak{s l}_{2}\right)$. Duke Math J., 87(3):409-480, 1997.

[24] K. Fukaya. Floer homology for 3-manifolds with boundary. In Topology geometry and field theory, pages 1-21. World Sci. Publishing, River Edge, NJ, 1994.

[25] K. Fukaya and P. Seidel. Floer homology, $A_{\infty}$-categories and topological field theory. In Geometry and physics (Aarhus, 1995), Lecture notes in Pure and Appl. Math., pages 9-32. Dekker, New York, 1997.

[26] D. Happel. Triangulated categories in the representation theory of finitedimensional algebras. London Math. Soc. Lect. Note Ser. 119. Cambridge University Press, 1988.

[27] R. S. Huerfano and M. Khovanov. A category for the adjoint representation. arXiv:math.QA/0002060.

[28] B. Iversen. Cohomology of sheaves. Springer-Verlag, 1987. 
[29] J. C. Jantzen. Representations of algebraic groups. Pure and Appl. Math. 131. Academic Press, Inc., Boston, 1987.

[30] V. F. R. Jones. A polynomial invariant for knots via von Newmann algebras. Bull. AMS, 12(1):103-111, 1985.

[31] L. Kadison. New examples of Frobenius extensions. University lecture series 14. AMS, 1999.

[32] M. Kapranov and E. Vasserot. Kleinian singularities, derived categories, and Hall algebras. arXiv:math.AG/9812016

[33] M. Kashiwara and P. Schapira. Sheaves on manifolds. Grundlehren der mathematischen Wissenschaften 292. Springer-Verlag, 1990.

[34] L. H. Kauffman. State models and the Jones polynomial. Topology, 26(3):395407, 1987.

[35] L. H. Kauffman and S. L. Lins. Temperley-Lieb recoupling theory and invariants of 3-manifolds. Annals of Math. Studies, 134. Princeton University Press, 1994.

[36] M. Khovanov. NilCoxeter algebras categorify the Weyl algebra. To appear in Communications in Algebra. arXiv:math.RT/9906166

[37] M. Khovanov. Graphical calculus, canonical bases and Kazhdan-Lusztig theory. $\mathrm{PhD}$ thesis, Yale University, 1997.

[38] M. Khovanov. A categorification of the Jones polynomial. Duke Math J., 101(3):359-426, 1999. arXiv:math.QA/9908171

[39] M. Khovanov and P. Seidel. Quivers, Floer homology, and braid group actions. arXiv:math.QA/0006056

[40] A. N. Kirillov and N. Y. Reshetikhin. Representations of the algebra $U_{q}(\operatorname{sl}(2))$, q-orthogonal polynomials and invariants of links. In V.G.Kac, editor, Infinite dimensional Lie algebras and groups. World Scientific, 1989.

[41] M. Kontsevich. Homological algebra of mirror symmetry. In Proceedings of the International Congress of Mathematicians, (Zürich, 1994), pages 120-139. Birkhäuser, 1995. arXiv:math. AG/9411018

[42] G. Kuperberg. Spiders for rank 2 Lie algebras. Comm. Math. Phys., 180(1):109151, 1996. arXiv:math.QA/9712003

[43] T. Y. Lam. Lectures on modules and rings. Graduate texts in mathematics 189. Springer-Verlag, 1999.

[44] M. Mackaay. Finite groups, spherical 2-categories, and 4-manifold invariants. arXiv:math.QA/9903003

[45] G. Malle and A. Mathas. Symmetric cyclotomic Hecke algebras. Journal of Algebra, 205:275-293, 1998.

[46] H. Nakajima. Instantons on ALE spaces, quiver varieties and Kac-Moody algebras. Duke Mathematical Journal, 76:365-416, 1994. 
[47] A. Polishchuk. Gluing of perverse sheaves on the basic affine space. arXiv:math.AG/9811155

[48] N. Reshetikhin and V. Turaev. Invariants of 3-manifolds via link polynomials and quantum groups. Invent. Math., 103(3):547-597, 1991.

[49] J. Rickard. Triangulated categories in the modular representation theory of finite groups. In Derived equivalences for group rings, volume 1685 of Lecture Notes in Math., pages 177-198. Springer-Verlag, 1998.

[50] R. Rouquier. Action du groupe de tresses sur la catégorie dériveé de la variété de drapeaux. preprint, http://www.math.jussieu.fr/ rouquier/

[51] R. Rouquier. Travaux de recherches. Représentations et catégories dérivées. http://www.math.jussieu.fr/〜rouquier/, 1998.

[52] R. Rouquier and A. Zimmermann. Picard groups for derived module categories. preprint, http://www.math.jussieu.fr/ ºuquier/, 1998.

[53] P. Seidel and R. Thomas. Braid group actions on derived categories of coherent sheaves. Duke Mathematical Journal, to appear, 2000. arXiv:math. AG/0001043

[54] M. E. Sweedler. Hopf algebras. W. A. Benjamin, Inc., New York, 1969.

[55] V. Turaev. Quantum invariants on knots and 3-manifolds. de Gruiter studies in mathematics, 18. 1994.

[56] W. Wang. Hilbert schemes, wreath products, and the Mckay correspondence. arXiv:math.AG/9912104

[57] E. Witten. Quantum field theory and the Jones polynomial. Comm. Math. Phys., 121(3):351-399, 1989.

Department of Mathematics, University of California

Davis, CA 95616, USA

Email: mikhail@math.ucdavis.edu

Received: 21 February 2002

Algebraic 63 Geometric Topology, Volume 2 (2002) 